\title{
ALGUNS MODELOS PARA DADOS DE CONTAGEM RESULTANTES DE EXPERIMENTOS COM CULTURA DE TECIDOS
}

\author{
ANA MARIA FIORAVANTE \\ Engenheiro Agrônomo
}

Orientadora: Prof $^{a}$. Dr ${ }^{a}$. CLARICE G. B. DEMÉTRIO

Dissertação apresentada à Escola Superior de. Agricultura "Luiz de Queiroz", da Universidade de São Paulo, para obtenção do título de Mestre em Agronomia, Área de Concentração: Estatística e Experimentação Agronômica.

P I R A C I C A B A

Estado de São Paulo - Brasil

Outubro - 1995 
Dados Internacionais de Catalogação na Publicação (CIP)

DIVISÃo DE BIBLIOTECA E DOCUMENTAÇÃO - Campus “Luiz de Queiroz"/USP

\section{Fioravante, Ana Maria}

Alguns modelos para dados de contagem resultantes de experimentos

com cultura de tecidos / Ana Maria Fioravante. - - Piracicaba, 1995.

$136 p$.

Dissertação (mestrado) - - Escola Superior de Agricultura Luiz de Queiroz, 1996. Bibliografia.

1. Cultura de tecido - Modelo matemático 2. Delineamento de experimento

3. Modelo matemático I. Título

CDD $\quad 515.4$ $\mathbf{5 7 4 . 8 2 4}$ 


\section{ALGUNS MODELOS PARA DADOS DE CONTAGEM RESULTANTES DE EXPERIMENTOS COM CULTURA DE TECIDOS}

ANA MARIA FIORAVANTE

Aprovada em: 30.01.1996

Comissão julgadora:

$\operatorname{Prof}^{a}$. Dr ${ }^{a}$. Clarice Garcia Borges Demétrio

ESALQ/USP

Prof. Dr. Décio Barbin

ESALQ/USP

Prof $^{a}$. Dr ${ }^{a}$. Sheila Zambello de Pinho

IB/UNESP

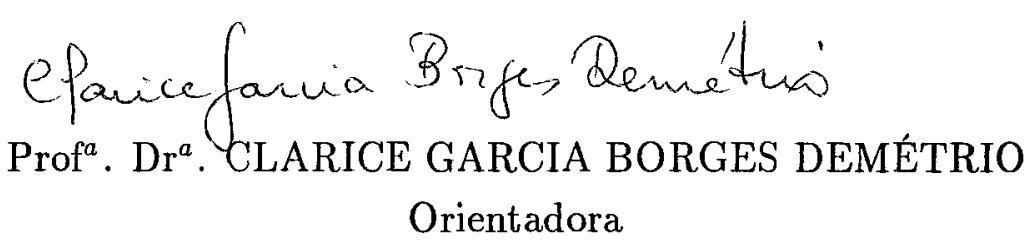


A meus pais,

Henrique e Leonela,

A meus irmãos,

José Henrique, Paulina e Ricardo,

A minhas queridas sobrinhas, Isabella e Anna Beatriz,

dedico. 


\section{AGRADECIMENTOS}

A Professora $\operatorname{Dr}^{a}$. Clarice Garcia Borges Demétrio, pela orientação e pela amizade.

A Professora $\operatorname{Dr}^{a}$. Sheila Zambello de Pinho e ao Professor Dr. Décio Barbin, por suas sugestões.

A amiga e colega María del Pilar Díaz, pelas valiosas discussões, pela confiança e pelo apoio.

A $\operatorname{Dr}^{a}$. Maria Lúcia Carneiro Vieira, professora do Departamento de Genética da ESALQ/USP, e a Marcelo Carnier Dornelas, aluno de pós-graduação do mesmo Departamento, pelos dados fornecidos e pela especial atenção dispensada.

Ao Dr. Fernando Berlinck d'Utra Vaz e à Dr ${ }^{a}$. Beatriz Madalena Januzi Mendes, professores do Centro de Energia Nuclear na Agricultura (CENA), pelos dados fornecidos e pela disposição em colaborar.

A Otto Ricardo Pieske, aluno de graduação da ESALQ/USP e estagiário do Departamento de Matemática e Estatística, pela ajuda na confecção dos gráficos e por sua gentileza durante os trabalhos.

Ao Professor Dr. José Eduardo Corrente, pelo incentivo e por sua atenção.

A Professora Roseli Aparecida Leandro, pela convivência e pela amizade.

A Coordenação de Aperfeiçoamento de Pessoal de Nivel Superior (CAPES), pela bolsa de estudos concedida.

A bibliotecária Kátia Maria de Andrade Ferraz, por sua colaboração. 
Aos funcionários Rosa Maria Alves, Solange Sabadin, Rosni Honofre Aparecido Pinto, Luciane Brajão e Expedita Maria de Azevedo, pelos auxílios prestados.

Aos professores do Departamento de Matemática e Estatística da ESALQ/USP e a todos os colegas, pelos ensinamentos e pelo companheirismo. 
SUMÁRIO

Página

LISTA DE FIGURAS $\ldots \ldots \ldots \ldots \ldots \ldots \ldots \ldots \ldots \ldots \ldots \ldots \ldots \ldots \ldots \ldots \ldots \ldots \ldots \ldots$

LISTA DE TABELAS $\ldots \ldots \ldots \ldots \ldots \ldots \ldots \ldots \ldots \ldots \ldots \ldots \ldots \ldots \ldots \ldots \ldots \ldots \ldots \ldots \ldots$

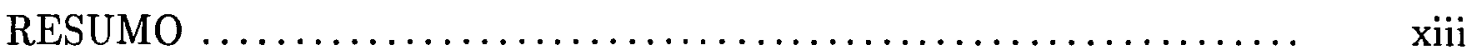

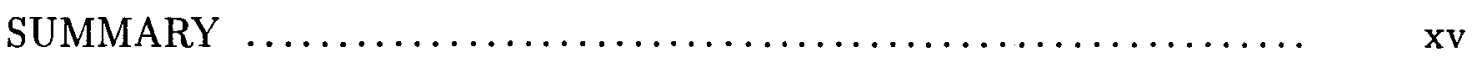

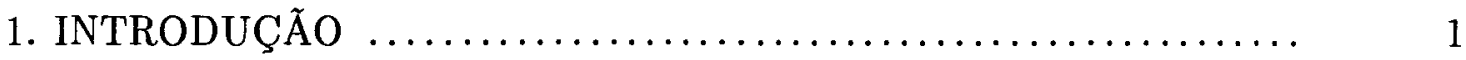

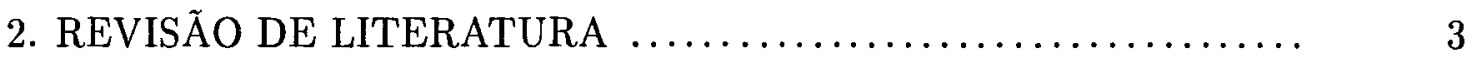

2.1 A teoria de modelos lineares generalizados ................ 3

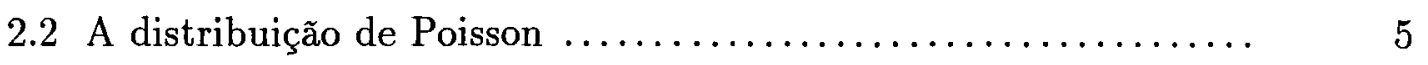

2.3 A distribuição Poisson truncada $\ldots \ldots \ldots \ldots \ldots \ldots \ldots \ldots \ldots \ldots$

2.4 A distribuição Poisson inflacionada de zeros ............... 12

2.5 As distribuições Poisson compostas e a distribuição binomial ne-

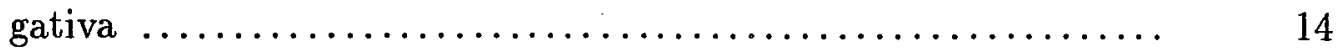

2.6 Teste do cociente variância-média para a distribuição dos dados $\quad 18$

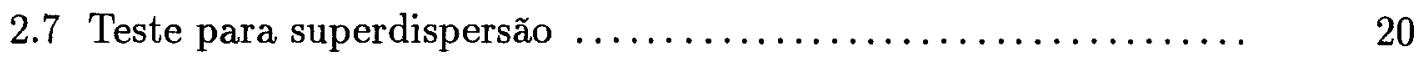

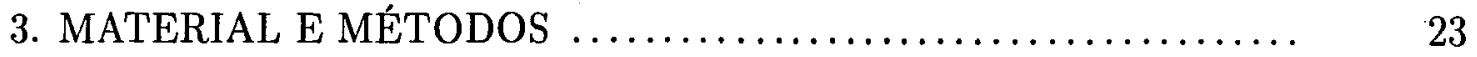

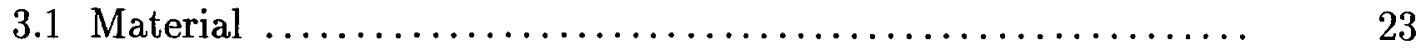

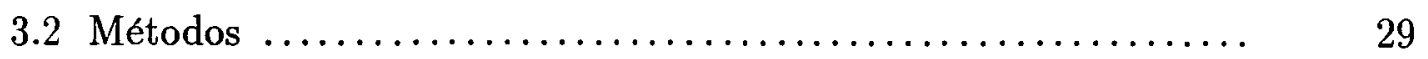

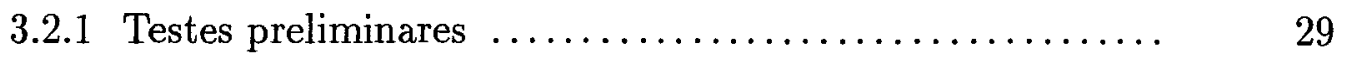

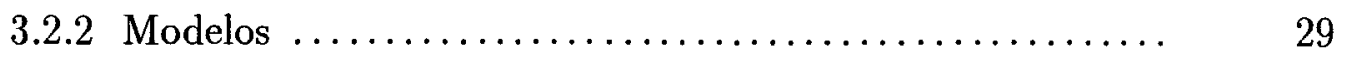

3.2.2.1 Modelo Poisson ....................... 29

3.2.2.2 Modelo Poisson truncado ............... 31

3.2.2.3 Modelo binomial negativo ............... 32

3.2.3 Técnicas para verificação do ajuste do modelo ......... 33

3.2.3.1 Análise de "deviance" ................. 33

3.2.3.2 Análise de resíduòs e diagnósticos ........... 35

4. RESULTADOS E DISCUSSÃO $\ldots \ldots \ldots \ldots \ldots \ldots \ldots \ldots \ldots \ldots \ldots \ldots \ldots \ldots \ldots \ldots$

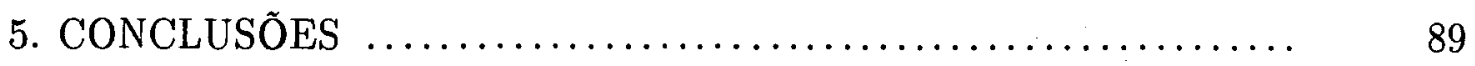

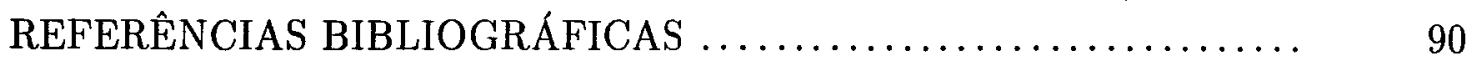

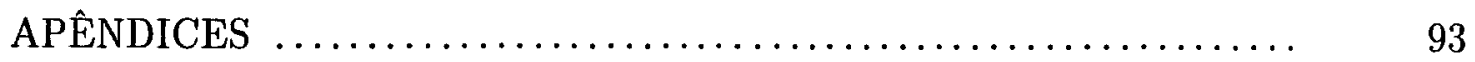




\section{LISTA DE FIGURAS}

Página

1. Gráfico dos componentes de "deviance" padronizados versus índices, resultante do ajuste do modelo Poisson ao Conjunto A de da-

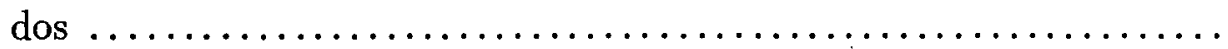

2. Gráfico dos componentes de "deviance" estudentizados externamente versus índices, resultante do ajuste do modelo Poisson ao Con-

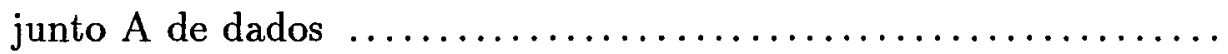

3. Gráfico dos componentes de "deviance" padronizados versus tratamentos ordenados pelas médias, resultante do ajuste do modelo Poisson ao Conjunto A de dados $\ldots \ldots \ldots \ldots \ldots \ldots \ldots \ldots \ldots \ldots$

4. Gráfico normal de probabilidade para os valores absolutos dos componentes de "deviance" padronizados, resultante de ajuste do modelo Poisson ao Conjunto $\mathrm{A}$ de dados $\ldots \ldots \ldots \ldots \ldots \ldots \ldots \ldots \ldots$

5. Gráfico dos componentes de "deviance" padronizados versus indices, resultante do ajuste do modelo Poisson truncado ao Conjunto

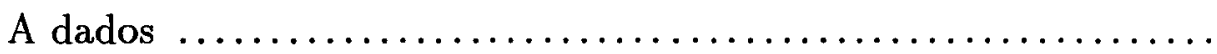

6. Gráfico dos componentes de "deviance" estudentizados externamente versus índices, resultante do ajuste do modelo Poisson truncado ao Conjunto $\mathrm{A}$ de dados $\ldots \ldots \ldots \ldots \ldots \ldots \ldots \ldots \ldots \ldots \ldots \ldots \ldots \ldots \ldots \ldots \ldots$

7. Gráfico dos componentes de "deviance" padronizados versus tratamentos ordenados pelas médias, resultante do ajuste do modelo

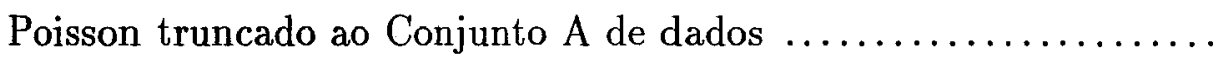

8. Gráfico dos valores observados e dos valores estimados para o número médio de calos produzidos por explante de Stylosanthes scabra, dado que o explante regenerou, na presença de lâmpada branca fria ou lâmpada de amplo espectro, em função da dose de ácido naftaleno-acético no meio de cultura $\ldots \ldots \ldots \ldots \ldots \ldots \ldots \ldots$

9. Gráfico da proporção de explantes regenerados versus dose de ácido naftaleno-acético no meio de cultura, em cada nível do fator tipo 
10. Gráfico da proporção de explantes regenerados versus tipo de radiação luminosa, em cada dose de ácido naftaleno-acético, para os tratamentos do Conjunto A de dados

11. Gráfico dos componentes de "deviance" padronizados versus índices, resultante do ajuste do modelo Poisson ao Conjunto B de dados

12. Gráfico dos componentes de "deviance" estudentizados externamente versus índices, resultante do ajuste do modelo Poisson ao Conjunto $\mathrm{B}$ de dados $\ldots \ldots \ldots \ldots \ldots \ldots \ldots \ldots \ldots \ldots \ldots \ldots . . \ldots \ldots \ldots$

13. Gráfico dos componentes de "deviance" padronizados versus tratamentos ordenados pelas médias, resultante do ajuste do modelo Poisson ao Conjunto $\mathrm{B}$ de dados $\ldots \ldots \ldots \ldots \ldots \ldots \ldots \ldots \ldots$

14. Gráfico normal de probabilidade para os valores absolutos dos componentes de "deviance" padronizados, resultante de ajuste do modelo Poisson ao Conjunto $\mathrm{B}$ de dados $\ldots \ldots \ldots \ldots \ldots \ldots \ldots \ldots$

15. Gráfico dos componentes de "deviance" padronizados versus índices, resultante do ajuste do modelo Poisson truncado ao Conjunto

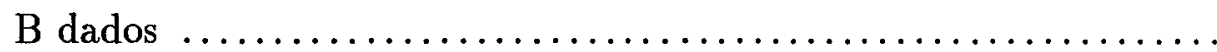

16. Gráfico dos componentes de "deviance" estudentizados externamente versus índices, resultante do ajuste do modelo Poisson truncado

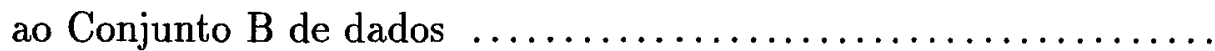

17. Gráfico dos componentes de "deviance" padronizados versus tratamentos ordenados pelas médias, resultante do ajuste do modelo Poisson truncado ao Conjunto $\mathrm{B}$ de dados $\ldots \ldots \ldots \ldots \ldots \ldots \ldots$

18. Gráfico dos componentes de "deviance" padronizados versus índices, resultante do ajuste do modelo binomial negativo ao Conjunto

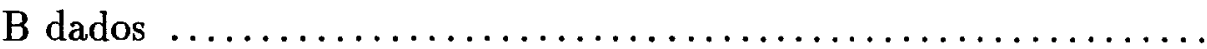

19. Gráfico dos componentes de "deviance" estudentizados externamen- 
te versus índices, resultante do ajuste do modelo binomial negativo

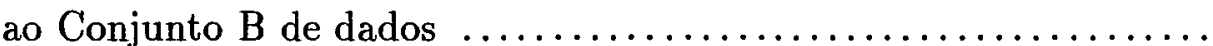

20. Gráfico dos componentes de "deviance" padronizados versus tratamentos ordenados pelas médias, resultante do ajuste do modelo binomial negativo ao Conjunto $\mathrm{B}$ de dados $\ldots \ldots \ldots \ldots \ldots \ldots$

21. Gráfico dos valores observados e dos valores estimados para o número médio de calos produzidos por explante de Stylosanthes scabra na presença de carvão ativado, em função da concentração de água de coco no meio de cultura $\ldots \ldots \ldots \ldots \ldots \ldots \ldots \ldots \ldots$

22. Gráfico dos componentes de "deviance" padronizados versus indices, resultante do ajuste do modelo Poisson ao Conjunto $\mathrm{C}$ de da-

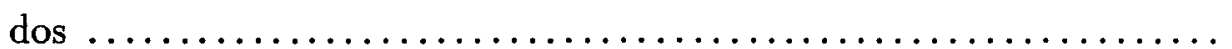

23. Gráfico dos componentes de "deviance" estudentizados externamente versus índices, resultante do ajuste do modelo Poisson ao Con-

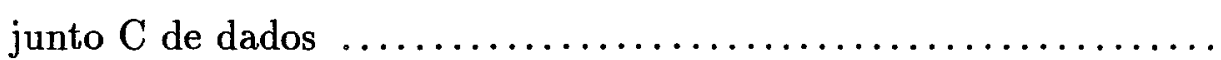

24. Gráfico dos componentes de "deviance" padronizados versus tratamentos ordenados pelas médias, resultante do ajuste do modelo Poisson ao Conjunto $\mathrm{C}$ de dados $\ldots \ldots \ldots \ldots \ldots \ldots \ldots \ldots \ldots \ldots \ldots \ldots$

25. Gráfico normal de probabilidade para os valores absolutos dos componentes de "deviance" padronizados, resultante do ajuste do modelo Poisson ao Conjunto $\mathrm{C}$ de dados $\ldots \ldots \ldots \ldots \ldots \ldots \ldots \ldots$ 


\section{LISTA DE TABELAS}

Página

1. Teste do cociente variância-média aplicado aos tratamentos do Con-

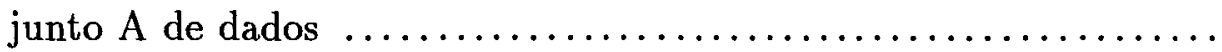

2. Resultados da análise de "deviance" para a variável número de calos produzidos por explante, do Conjunto A de dados, usando o

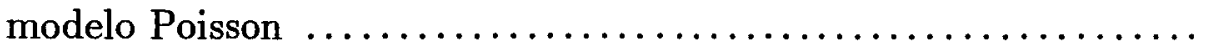

3. Resultados da análise de "deviance" para a variável número de calos produzidos por explante, do Conjunto A de dados, usando o modelo Poisson truncado

4. Resultados da análise de "deviance" para a variável número de calos produzidos por explante, do Conjunto A de dados, para estudo dos efeitos principais dos fatores, usando o modelo Poisson truncado

5. Médias de tratamentos do Conjunto $\mathrm{A}$ de dados para a variável número de calos produzidos por explante, dado que o explante regenerou, acompanhadas dos números de repetições dos tratamentos $(n)$ e de seus valores estimados $\left(\hat{\mu}_{1 j}\right) \ldots \ldots \ldots \ldots \ldots \ldots \ldots \ldots \ldots \ldots \ldots \ldots$

6. Número de explantes regenerados $(r)$, número total de explantes $(n)$ e proporção de explantes regenerados $(p=r / n)$, para os trata-

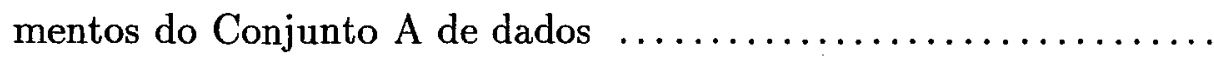

7. Resultados da análise de "deviance" para a variável proporção de explantes regenerados, do Conjunto A de dados, usando o modelo binomial

8. Teste do cociente variância-média aplicado aos tratamentos do Conjunto $\mathrm{B}$ de dados

9. Resultados da análise de "deviance" para a variável número de calos produzidos por explante, do Conjunto B de dados, usando o 


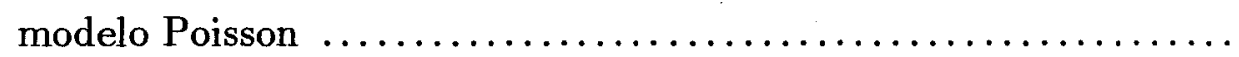

10. Resultados da análise de "deviance" para a variável número de calos produzidos por explante, do Conjunto B de dados, usando o

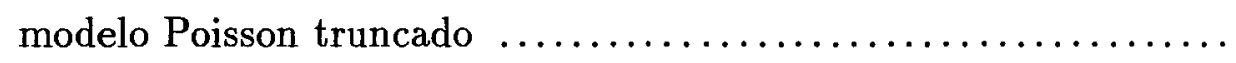

11. Resultados da análise de "deviance" para a variável número de calos produzidos por explante, do Conjunto $\mathrm{B}$ de dados, usando o modelo binomial negativo $\ldots \ldots \ldots \ldots \ldots \ldots \ldots \ldots \ldots \ldots \ldots \ldots \ldots \ldots \ldots \ldots \ldots \ldots$

12. Médias dos tratamentos do Conjunto $\mathrm{B}$ de dados para a variável número de calos produzidos por explante, acompanhadas dos números de repetiçôes dos tratamentos $(n) \ldots \ldots \ldots \ldots \ldots \ldots \ldots \ldots$

13. Resultados da análise de "deviance" para a variável número de calos produzidos por explante, do Conjunto B de dados, com desdobramento das interações, usando o modelo binomial negativo .....

14. Resultados da análise de "deviance" para a variável número de calos produzidos por explante, do Conjunto B de dados, com desdobramento das interações em efeitos linear e quadrático, usando o modelo binomial negativo $\ldots \ldots \ldots \ldots \ldots \ldots \ldots \ldots \ldots \ldots \ldots$

15. Médias de tratamentos do Conjunto $\mathrm{B}$ de dados para a variável número de calos produzidos por explante, acompanhadas de seus

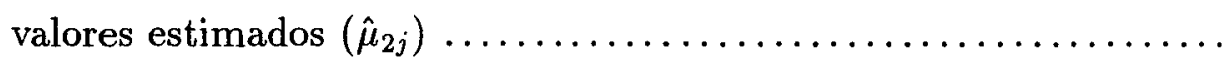

16. Teste do cociente variância-média aplicado aos tratamentos do Con-

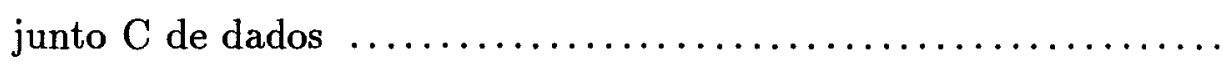

17. Resultados da análise de "deviance" para a variável número de calos produzidos por explante, do Conjunto $\mathrm{C}$ de dados, usando o

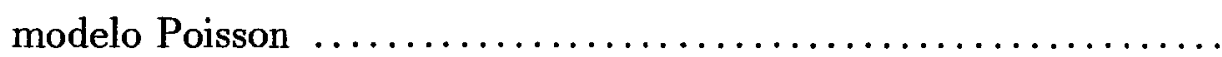

18. Resultados da análise de "deviance" para as médias das sub-amostras das repetições do Conjunto $\mathrm{C}$ de dados, variável número de calos produzidos por explante, usando o modelo Poisson ..........

19. Tratamentos do Conjunto $\mathrm{A}$ de dados ordenados pelas médias 
do número de calos produzidos por explante, e número de repeti-

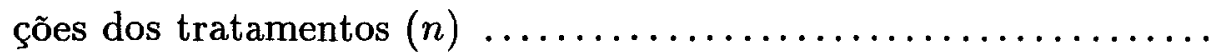

20. Tratamentos do Conjunto $A$ de dados ordenados pelas médias do número de calos produzidos por explante, dado que o explante regenerou, e número de repetições dos tratamentos $(n) \ldots \ldots \ldots$.

21. Tratamentos do Conjunto $B$ de dados ordenados pelas médias do número de calos produzidos por explante, e número de repeti-

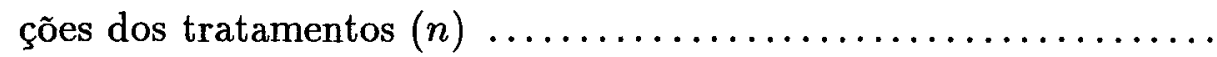

22. "Deviance" residual do modelo binomial negativo ajustado ao Conjunto $\mathrm{B}$ de dados, para diferentes valores do parâmetro $k$. (Saída do programa apresentado no Apêndice 8$) \ldots \ldots \ldots \ldots \ldots \ldots$

23. Médias do número de calos produzidos por explante $(m)$ para as placas, ou repetições, dos tratamentos do Conjunto $\mathrm{C}$ de dados ...

24. Tratamentos do Conjunto $\mathrm{C}$ de dados ordenados pelas médias do número de calos produzidos por explante, e número de repeti-

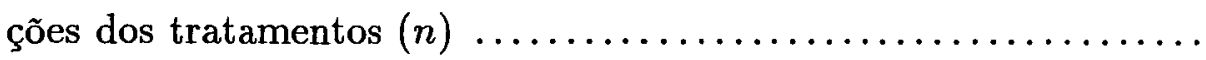




\title{
ALGUNS MODELOS PARA DADOS DE CONTAGEM RESULTANTES DE EXPERIMENTOS COM CULTURA DE TECIDOS
}

\author{
Autora: ANA MARIA FIORAVANTE \\ Orientadora: PROF ${ }^{a}$. DR ${ }^{a}$. CLARICE GARCIA BORGES DEMÉTRIO
}

\section{RESUMO}

Em experimentos com cultura de tecidos é comum a obtenção de dados de contagem, como o número de calos, de brotos ou de rafizes produzidos por explante. Pode-se admitir, a princípio, que dados de contagem seguem a distribuição de Poisson. Neste trabalho, utilizando o enfoque de modelos lineares generalizados, são apresentados os modelos Poisson, Poisson truncado e binomial negativo para a análise de dados de contagem resultantes de experimentos com cultura de tecidos. Essas contagens, freqüentemente, apresentam o número de observações iguais a zero maior do que seria esperado com base no número de calos, por exemplo, produzidos pelos explantes que regeneram. Outro problema que esses dados podem apresentar é o fenômeno conhecido como superdispersão, que significa que a variância das observações é maior que sua média. Quando essas situações ocorrem, o modelo Poisson, admitido a princípio, não se ajusta bem às observações, e os modelos Poisson truncado e binomial negativo são apresentados como alternativas para esse problema.

Como aplicação, foram utilizados três conjuntos de dados, aqui designados por Conjuntos $\mathrm{A}, \mathrm{B}$ e $\mathrm{C}$, e a variável resposta estudada foi o número de calos produzidos por explante.

Os Conjuntos A e B são resultantes de dois experimentos fatoriais, $3^{2}$ e $2 \times 7$, respectivamente, instalados no delineamento inteiramente casualizado e referem-se ao número de calos produzidos por explante de Stylosanthes scabra, 
espécie leguminosa usada como forrageira, mantido em meio de cultura por vinte e oito dias. Os fatores estudados nesses experimentos são relativos à composição do meio de cultura e ao tipo de radiação luminosa a que a cultura fica exposta. $\mathrm{O}$ Conjunto $\mathrm{C}$ de dados é resultante de um experimento fatorial $3 \times 4$ instalado no delineamento inteiramente casualizado e refere-se ao número de calos produzidos por explante. A espécie doadora do explante, pertencente ao gênero Passiflora (maracujá), foi um dos fatores estudados neste experimento, sendo o outro fator relativo à composição do meio de cultura.

Entre os modelos Poisson e Poisson truncado, este foi o que melhor se ajustou ao Conjunto $\mathrm{A}$ de dados, que não apresentou superdispersão. Para o Conjunto B de dados, que apresentou superdispersão, o melhor modelo foi o binomial negativo, e para o Conjunto $\mathrm{C}$, que também não apresentou superdispersão, o modelo Poisson ofereceu um bom ajuste. 


\section{SOME MODELS FOR COUNT DATA FROM TISSUE CULTURE EXPERIMENTS}

Author: ANA MARIA FIORAVANTE Adviser: PROF ${ }^{a}$. DR ${ }^{a}$. CLARICE GARCIA BORGES DEMÉTRIO

\section{SUMMARY}

In tissue culture experiments is common to have count data, like the number of proliferated callus, shoots or roots by explant. In principle, we can assume that count data follows the Poisson distribution. In this work, using the generalized linear models theory, the Poisson, the truncated Poisson and the negative binomial models are presented for analysis of count data from tissue culture experiments. These counts often presents the observed number of zeros greater than would be expected on the basis of the non-zeros counts. Other problem that these counts can present is the phenomenum known as overdispersion, which means that the observations variance is greater than their mean. When these situations occur, the Poisson model does not fit well to the observations, and the truncated Poisson and the negative binomial models are presented like alternatives for this problem.

As an application, three sets of real data were used, here referred to as sets $A, B$ and $C$, and the studied response variable was the number of proliferated callus by explant.

The $\mathrm{A}$ and $\mathrm{B}$ sets of data come from two factorial experiments, $3^{2}$ and $2 \times 7$, respectively, in the completely randomized design, and refer to the number of proliferated callus by explant of Stylosanthes scabra, a leguminous species used as forage, maintained in culture medium for twenty-eight days. The studied factors in these experiments are related to the composition of the culture medium and to the type of light radiation the culture is exposed to. The $\mathrm{C}$ data set comes from a $3 \times 4$ 
factorial experiment in the completely randomized design and refer to the number of proliferated callus by explant. The giving species of the explant, belonging to the Passiflora genus (passion fruit), was one of the studied factors in this experiment, and the other factor is related to the composition of the culture medium.

Between the Poisson and the truncated Poisson models, the truncated Poisson was the one that fitted better to the A data set, which did not presented overdispersion. The model that fitted better to the B set of data, which presented overdispersion, was the negative binomial model, and to the $\mathrm{C}$ set of data, which did not present overdispersion either, the Poisson model fitted well. 


\section{INTRODUÇÃO}

A cultura de tecidos é uma prática que, em virtude de sua própria natureza, constitui-se numa grande ferramenta para o conhecimento e a utilização dos recursos biológicos, fato que, naturalmente, confere-lhe grande importância na pesquisa científica. Esta prática consiste na cultura "in vitro" de células ou fragmentos de tecidos vegetais ou animais com o objetivo de se obter a regeneração dessas células ou desses fragmentos. Em função das alterações que se pode fazer no meio de cultura e nas condições a que a cultura fica exposta, são múltiplas as maneiras de se instalar e conduzir uma cultura de tecidos ${ }^{1}$, assim como são múltiplas as diferentes aplicações que esta prática encontra.

No que diz respeito à cultura de tecidos de plantas, suas aplicações mais expressivas encontram-se no melhoramento e na produção de espécies vegetais, sendo consagrada sua importância no desenvolvimento dessas atividades. Conseqüentemente, os experimentos que envolvem a cultura de tecidos são bastante utilizados na pesquisa cientffica agronômica.

Dentre os diversos tipos de dados obtidos em experimentos com cultura de tecidos, estão a porcentagem de explantes regenerados e a contagem do número de calos, de brotos e de rá́zes produzidos por explante, os quais, em geral, têm sido analisados com base no modelo clássico com erro normal após terem sido transformados segundo transformações do tipo $\sqrt{Y+c}, \log (Y+c)$, e arco seno $\sqrt{X / 100}$,

\footnotetext{
${ }^{1} \mathrm{O}$ termo "cultura de tecidos" foi estabelecido quando esta prática se retringia à cultura de tecidos somente, mas com o desenvolvimento da técnica o termo passou a englobar a cultura de células, protoplastos (células vegetais desprovidas de parede), fragmentos de órgãos, e embriões. As pequenas partes extrafdas da planta para serem cultivadas "in vitro" foram denominadas explantes.
} 
onde $c$ representa uma constante positiva, e $Y$ e $X$ representam os dados observados e referem-se, respectivamente, a dados de contagem e a dados de porcentagem.

Pode-se considerar, a princípio, que dados de contagem tomados num intervalo contínuo (tempo, área, etc.) seguem a distribuição de Poisson e que dados de proporções seguem a distribuição binomial. O objetivo deste trabalho é apresentar modelos alternativos para dados de contagem resultantes de experimentos com cultura de tecidos adotando-se a teoria de modelos lineares generalizados e partindose das distribuições Poisson, Poisson truncada, e binomial negativa, sendo esta um caso particular de distribuição Poisson composta. 


\section{REVISÃO DE LITERATURA}

\subsection{A teoria de modelos lineares generalizados}

Os modelos lineares generalizados são uma extensão dos modelos lineares clássicos proposta por NELDER \& WEDDERBURN (1972).

São bem conhecidas as pressuposições de independência, normalidade, e variância constante das observações do modelo linear clássico. Pela teoria de modelos lineares generalizados, estas duas últimas pressuposições são afrouxadas, pois, neste caso, admite-se qualquer distribuição pertencente à famólia exponencial para as observações e, quanto a sua variância, a exigência é que esta tenha relação conhecida com a média (McCullaGH \& NELDER, 1989).

Define-se modelo linear generalizado como o modelo em que se têm:

- um componente aleatório representado pela variável aleatória $Y$ que descreve as observações, onde $Y$ tem distribuição pertencente à família exponencial na forma canônica;

- um componente sistemático linear nos parâmetros, ou preditor linear, representado por $\eta$ e tal que

$$
\eta=X \beta
$$

onde $\eta$ é um vetor $n \mathrm{x} 1, n$ o número de observações; $X$ é a matriz do modelo de dimensão $n \times p, p$ o número de parâmetros desconhecidos do preditor linear; e $\beta$ é o vetor $p \times 1$ de parâmetros desconhecidos;

- uma função de ligação $g(\cdot)$ monótona e diferenciável tal que

$$
\eta_{i}=g\left(\mu_{i}\right) \Leftrightarrow \mu_{i}=g^{-1}\left(\eta_{i}\right)
$$


onde $\mu_{i}=E\left(Y_{i}\right), i=1,2, \ldots, n$.

Considerando-se a notação usada por MCCullaGH \& NELDER (1989) para as distribuições da família exponencial, a saber,

$$
f\left(y_{i} ; \theta_{i}, \phi\right)=\exp \left\{\frac{1}{a_{i}(\phi)}\left[y_{i} \theta_{i}-b\left(\theta_{i}\right)\right]+c\left(y_{i} ; \phi\right)\right\} I_{A}\left(y_{i}\right)
$$

onde $f(\cdot ; \cdot, \cdot)$ é uma função de probabilidade ou uma função densidade de probabilidade;

$y_{i}$ é o valor observado para a variável aleatória $Y_{i}$ com distribuição pertencente à famflia exponencial na forma canônica;

$\theta_{i}$ é o parâmetro natural ou canônico da distribuição; $a_{i}(\phi)=\phi / w_{i}$, sendo $\phi>0$ o parâmetro de escala da distribuição, suposto conhecido, e $w_{i}$ um peso a priori para a observação $y_{i}$;

$b(\cdot)$ e $c(\cdot ; \cdot)$ são funções conhecidas;

$A$ é o conjunto de todos os valores que $Y_{i}$ pode assumir e independe de $\theta_{i}$;

então verifica-se que, para a esperança e a variância de $Y_{i}$, as seguintes relações são verdadeiras (MCCUllagh \& Nelder, 1989):

$$
\begin{aligned}
& E\left(Y_{i}\right)=\mu_{i}=b^{\prime}\left(\theta_{i}\right) \\
& V\left(Y_{i}\right)=b^{\prime \prime}\left(\theta_{i}\right) a_{i}(\phi),
\end{aligned}
$$

onde $a_{i}(\phi)=\phi / w_{i}$.

Fica evidenciado na equação (3) que a variância de $Y_{i}$ é o produto de duas funções: uma, $b^{\prime \prime}\left(\theta_{i}\right)$, que depende do parâmetro canônico $\theta_{i}$, e conseqüentemente de $\mu_{i}$, chamada função de variância; e outra, $a_{i}(\phi)$, que depende somente de $\phi$, o parâmetro de escala ou parâmetro de dispersão. A função de variância, considerada como função de $\mu_{i}$, é denotada por $V\left(\mu_{i}\right)$, ou seja,

$$
V\left(\mu_{i}\right)=b^{\prime \prime}\left(\theta_{i}\right)
$$


As distribuições da familia exponencial, portanto, têm uma relação conhecida entre a média e a variância e, se $Y$ é uma variável aleatória com distribuiçâo pertencente à família exponencial, esta relação é dada por:

$$
V(Y)=V(\mu) \frac{\phi}{w}
$$

onde $\mu=E(Y)$. No caso da distribuição normal, tem-se $V(\mu)=1 \mathrm{e}$, portanto, $V(Y)$ independe de $\mu$ :

$\mathrm{O}$ ajuste de um modelo linear generalizado é determinado pela estimativa $\hat{\beta}$ 'do vetor $\beta$ de parâmetros desconhecidos do preditor linear, a qual é obtida pelo método da máxima verossimilhança e tem como algoritmo de estimação uma regressão ponderada iterativa (MCCULlaGH \& NELDER, 1989).

\subsection{A distribuição de Poisson}

A distribuição de Poisson é de grande importância na Estatística Experimental, pois consiste num modelo probabilístico adequado para um grande número de fenômenos observáveis. É o caso dos fenômenos que podem ser representados por uma contagem de eventos discretos num intervalo contínuo, onde tais eventos são gerados de modo a satisfazer a Definição 1 abaixo, dada por LARṢon, 1974.

Definição 1 Em um Processo de Poisson com parâmetro $\lambda$, eventos discretos são gerados num intervalo contínuo (tempo, área, etc.) de acordo com o seguinte:

- Pode-se tomar um intervalo suficientemente curto, de comprimento $h$, tal que:

- a probabilidade de ocorrer exatamente um evento neste intervalo é aproximadamente $\lambda h, \mathbf{e}$

- a probabilidade de ocorrerem dois ou mais eventos neste intervalo é aproximadamente zero; 
- A ocorrência de um evento em um intervalo de comprimento $h$ não tem efeito na ocorrência, ou não, de um evento em outro intervalo de comprimento $h$ não sobreposto ao primeiro. (As ocorrências são estatisticamente independentes entre si.)

Por exemplo, a chegada de insetos numa planta num intervalo de tempo determinado é um fenômeno que pode ser visto como um processo de Poisson, pois seria razoável assumir que não mais que 1 (um) inseto pousaria na planta em um décimo de segundo e que a ocorrência, ou não, da chegada de um inseto na planta no primeiro décimo de segundo não teria efeito sobre a ocorren̂cia, ou não, da chegada de um inseto na planta no próximo, ou em qualquer outro, décimo de segundo seguinte.

Observando-se um processo de Poisson num intervalo contínuo determinado, e considerando-se a dimensão desse intervalo como unitária, tem-se que o número de eventos que ocorrem neste intervalo unitário é uma variável aleatória. Ou seja, assumindo-se que num processo de Poisson os eventos ocorrem a uma taxa constante $\lambda$, não se pode afirmar que exatamente $\lambda$ eventos ocorrerão em um intervalo unitário, mas tem-se que a média do número de eventos que ocorrem por intervalo unitário é $\lambda$. Tal variável aleatória, demonstra-se que tem distribuição de Poisson.

A função de probabilidade de uma variável aleatória $Y$ com distribuição de Poisson com parâmetro $\lambda$ é dada por:

$$
P(Y=y)=\frac{\lambda^{y} e^{-\lambda}}{y !} I_{\{0,1,2, \ldots\}}(y), \quad \lambda>0
$$

e usa-se a notação

$$
Y \sim \operatorname{Poisson}(\lambda)
$$

A distribuição de Poisson pertence à família exponencial de distribuições, e considerando-se uma variável aleatória $Y_{i}$ com distrubuição de Poisson com parâmetro $\lambda_{i}$, sua função de probabilidade na forma apresentada pela 
equação (1) fica:

$$
f\left(y_{i} ; \lambda_{i}\right)=\exp \left\{\left[y_{i} \ln \lambda_{i}-\lambda_{i}\right]+\left(-\ln y_{i} !\right)\right\}
$$

onde $\ln \lambda_{i}=\theta_{i} ; \lambda_{i}=b\left(\theta_{i}\right)=e^{\theta_{i}} ;-\ln y_{i} !=c\left(y_{i} ; \phi\right) ; \phi=1 ;$ e $w_{i}=1$.

Aplicando-se na equação (6) os resultados expressos pelas equações (2) e (3), tem-se:

$$
\begin{aligned}
& E\left(Y_{i}\right)=\mu_{i}=b^{\prime}\left(\theta_{i}\right)=\lambda_{i} \\
& V\left(Y_{i}\right)=b^{\prime \prime}\left(\theta_{i}\right) \frac{\phi}{w_{i}}=\lambda_{i} .
\end{aligned}
$$

Portanto, na distribuição de Poisson existe uma relação de igualdade entre média e variância. De fato, o modelo Poisson proporciona, em geral, uma descrição satisfatória de dados experimentais de contagem cuja variância é proporcional à média e que representam o número de ocorrências independentes e aleatórias de um evento discreto num intervalo contínuo, evento este com taxa de ocorrência $\lambda$ constante.

Dados para os quais se admite a distribuição de Poisson podem ser submetidos a uma análise de regressão utilizando-se o modelo log-linear, que é um modelo de regressão para dados discretos análogo ao modelo usual de regressão normal para dados contínuos e que consiste num modelo linear para o logaritmo da média da variável observada, pois assume-se que a relação entre esta e as covariáveis do modelo é multiplicativa. Especificamente para o modelo de regressão Poisson em que a variável resposta $Y$ é explicada por um conjunto de variáveis explanatórias $X_{1}, X_{2}, \ldots, X_{p}$, tem-se:

$$
Y_{i} \mid X_{1 i}, X_{2 i}, \ldots, X_{p_{i}} \sim \operatorname{Poisson}\left(\mu_{i}\right)
$$

com

$$
\ln \left(\mu_{i}\right)=\beta_{0}+\beta_{1} X_{1 i}+\cdots+\beta_{p} X_{p_{i}}
$$

onde $o$ indice $i$ representa a i-ésima observação de $Y, i=1,2, \ldots, n$. Então, adotando-se a teoria de modelos lineares generalizados tem-se um modelo com: 
- distribuição de Poisson;

- função de ligação $g\left(\mu_{i}\right)=\eta_{i}=\ln \left(\mu_{i}\right)$; e

- preditor linear $\eta=X \beta$, onde $X$ é a matriz de variáveis explanatórias, de dimensão $n \times q, n$ o número de observações, $q=p+1$; e $\beta$ é o vetor $q \times 1$ de parâmetros desconhecidos.

Analogamente, para o modelo da análise da variância, em que se tem a variável resposta explicada por um conjunto de fatores em lugar das variáveis explanatórias do modelo de regressão, pode-se adotar o modelo acima descrito levando-se em consideração, no entanto, que neste caso a matriz $X$ que gera o preditor linear é a matriz do delineamento.

\subsection{A distribuição Poisson truncada}

O fenômeno produção de calos num explante, observado em experimentos com cultura de tecidos e também chamado regeneração, quando observado num intervalo de tempo definido pode ser avaliado pela contagem do número de calos produzidos pelo explante. Portanto, é de se admitir, a princípio, que dados referentes a este fenômeno sejam bem explicados pelo modelo Poisson. No entanto, verifica-se que, na prática, este modelo nem sempre oferece um bom ajuste para tais dados. Ridout \& DEMÉtrio (1992) atribuem essa falta de ajuste ao número de explantes não regenerados, ou seja, ao número de zeros observados, o qual se apresenta maior do que seria esperado com base na taxa de produção de calos dos explantes que regeneraram.

A distribuição de Poisson assume que a probabilidade de um evento ocorrer num determinado intervalo é independente do fato de o evento já ter ocorrido previamente ou não. CAMPBELl et al. (1991), ao estudarem o método de contracepção do anel vaginal em 1005 mulheres, verificaram que aquelas que tiveram uma primeira expulsão involuntária do anel têm mais predisposição para sofrer 
uma expulsão no próximo intervalo observado do que aquelas que não sofreram a primeira expulsão. Isso sugere a ocorrência de dois processos: um que determina se uma mulher é suscetível à primeira expulsão, e outro que, dado que uma mulher é suscetf́vel, determina, então, a sua taxa de expulsão. Neste caso, quando se utilizou o modelo Poisson, também não se obteve um bom ajuste.

Uma alternativa para esses casos é assumir que, uma vez que o evento em estudo foi observado uma primeira vez, então o número de eventos que ocorrem é distribuído segundo a distribuição Poisson truncada, também conhecida na literatura como Poisson condicional (CoHEN, 1960) ou Poisson positiva (JoHnson \& KoTZ, 1969), a qual consiste na distribuição de Poisson com os zeros excluídos e tem função de probabilidade dada por:

$$
P(Y=y \mid Y>0)=\frac{e^{-\lambda} \lambda^{y}}{y !\left(1-e^{-\lambda}\right)} I_{\{1,2, \ldots\}}(y), \quad \lambda>0 .
$$

Pode-se verificar facilmente que esta distribuição pertence à família exponencial com parâmetro de escala, $\phi$, igual a 1 ; peso a priori, $w$, igual a 1 ; $b(\theta)=\ln \left(e^{\lambda}-1\right)$; e parâmetro canônico, $\theta$, igual a $\ln (\lambda)$. Portanto, pode-se adotar a teoria de modelos lineares generalizados na análise de dados para os quais se admite a distribuição Poisson truncada. Porém, como mostram RIDOUT \& DEMÉTRIO (1992), é necessário que se introduzam modificações no algoritmo de estimação dos parâmetros deste modelo, pois, se $Y$ é uma variável aleatória com distribuição Poisson truncada com parâmetro $\lambda$, então a esperança e a variância de $Y$ são dadas por:

$$
\begin{gathered}
E(Y)=\mu=\frac{\lambda}{\left(1-e^{-\lambda}\right)}, \mathrm{e} \\
V(Y)=\frac{\lambda}{\left(1-e^{-\lambda}\right)}\left(1-\frac{\lambda e^{-\lambda}}{\left(1-e^{-\lambda}\right)}\right)=\mu(1+\lambda-\mu),
\end{gathered}
$$

de forma que, em relação ao modelo Poisson, tem-se um parâmetro a mais no modelo, pois a esperança de $Y$, $\mu$, é uma função do parâmetro $\lambda$ da distribuição. 
Adotando-se a ligação natural ou canônica, ou seja, $\eta=\theta$, tem-se um modelo com função de ligação

$$
\eta=\ln (\lambda)=g[h(\mu)]=g_{1}(\mu) .
$$

No entanto, não se pode obter, explicitamente, o parâmetro $\lambda$ em função de $\mu$, ou seja, $\lambda=h(\mu)$, pois

$$
\lambda=\mu\left(1-e^{-\lambda}\right) .
$$

RIDOUT \& DEMÉTRIo (1992) propõem um método para a obtenção de $\lambda$. Escrevendo-se a equação (11) na forma

$$
f[h(\mu)]=\mu\left(1-e^{-h(\mu)}\right)-h(\mu)=0,
$$

o método consiste em se resolver a equação (12) para $h(\mu)$ pelo método de NewtonRaphson, partindo-se da aproximação inicial

$$
h^{(0)}(\mu)=\mu\left[1-\exp \left(\frac{1}{\mu}-\mu\right)\right],
$$

e tomando-se, para o parâmetro $\lambda$, a aproximação obtida para $h(\mu)$ na primeira iteração.

Na distribuição Poisson truncada tem-se que a variância é menor que a média, pois, seja $\lambda$ o parâmetro desta distribuição, então tem-se:

$$
\lambda>0 \Rightarrow 0<1-e^{-\lambda}<1,
$$

e, portanto, das equações (9) e (13) tem-se:

$$
\mu>\lambda \Rightarrow \lambda-\mu<0 .
$$

Por outro lado, dada uma variável aleatória $Y$ qualquer, tem-se, por definição, que

$$
V(Y)>0,
$$

de modo que, das equações (10) e (14) tem-se:

$$
0<1+\lambda-\mu<1
$$


e, portanto, se $Y$ é uma variável aleatória com distribuição Poisson truncada, tem-se que

$$
V(Y)=\mu(1+\lambda-\mu)<\mu
$$

Admitindo-se a distribuição Poisson truncada para o número de ocorrências de um evento em estudo, tem-se um modelo para a média do número de eventos que ocorrem, dado que ao menos um evento ocorreu. No caso da variável número de calos produzidos por explante, ao admitir-se a distribuição Poisson truncada para a mesma, tem-se um modelo para a média do número de calos produzidos, dado que o explante regenerou. Portanto, com base na ocorrência, ou não, de regeneração durante o período estudado, separam-se os explantes em dois grupos: um em que a regeneração ocorre segundo a distribuição Poisson truncada com parâmetro $\lambda$, e outro em que não ocorre regeneração. Quanto à porcentagem de explantes que regeneram, havendo interesse, é possivel efetuar, paralelamente, uma análise para estudar o efeito das fontes de variação do modelo sobre a proporção de explantes regenerados usando o modelo logístico de regressão.

Ou seja, considerando-se a variável aleatória $R$ que representa o número de explantes que regeneram num total de $n$ explantes, e admitindo-se que $R$ segue a distribuição binomial com parâmetro $\mu$, tem-se que a variável aleatória $P=R / n$, que representa a proporção de explantes que regeneram, tem distribuição binomial com parâmetro $\mu$ e função de probabilidade pertencente à famflia exponencial na forma canônica dada por:

$$
f(p ; \mu)=\exp \left\{n\left[p \ln \left(\frac{\mu}{1-\mu}\right)+\ln (1-\mu)\right]+\ln \left(\begin{array}{c}
n \\
n p
\end{array}\right)\right\} I_{A}(p)
$$

onde $A=\left\{\frac{0}{n}, \frac{1}{n}, \ldots, \frac{n}{n}\right\}$.

Então, pela teoria de modelos lineares generalizados é possível ajustar um modelo logístico para um conjunto de $m$ observações independentes de $P$, $p_{1}, p_{2}, \ldots, p_{m}$, adotando-se a função de ligação canônica, $\eta=\theta$, pois, neste caso, em que a distribuição considerada para os dados é a binomial, tem-se que a ligação 
canônica vem a ser o logito de $\mu$, ou seja,

$$
\eta=\ln \left(\frac{\mu}{1-\mu}\right),
$$

também chamada função de ligação logística.

\subsection{A distribuição Poisson inflacionada de zeros}

Outra alternativa para se analisarem dados de contagem aos quais o modelo Poisson não oferece um bom ajuste em razão do grande número de zeros observados é assumir a distribuição Poisson inflacionada de zeros para a variável em estudo, cuja função de probabilidade é dada por:

$$
P(Y=y)=\left\{\begin{array}{cll}
p+(1-p) e^{-\lambda} & , \text { para } & y=0 \\
\frac{(1-p) e^{-\lambda} \lambda^{y}}{y !} & , \text { para } & y=1,2, \ldots,
\end{array}\right.
$$

onde $p$ é a proporção de casos em que o evento sob estudo nunca ocorre. Ou seja, assume-se que, com probabilidade $p$, a única observação possível é 0 (zero), e, com probabilidade $(1-p)$, uma variável aleatória com distribuição Poisson $(\lambda)$ é observada. Por exemplo, seja o evento ocorrência de defeitos em uma peça produzida por determinado equipamento. Considerando-se que, quando o equipamento está perfeitamente regulado a ocorrência de defeitos é quase impossível e que, quando o equipamento está desregulado os defeitos podem ocorrer segundo uma distribuição de Poisson com parâmetro $\lambda$, tem-se uma distribuição Poisson inflacionada de zeros. Neste caso, o parâmetro $p$ da distribuição expressa a probabilidade de o equipamento estar no estado perfeito e o parâmetro $\lambda$ expressa a média do número de defeitos que ocorrem quando o equipamento está no estado imperfeito.

Pelo exposto no item 2.2, é de se admitir, a princípio, que o número de defeitos que ocorrem em uma peça produzida por determinado equipamento segue a distribuição de Poisson. Seja $\lambda$ o parâmetro desta distribuiçãa, então, de acordo com este modelo, em uma grande amostra de $n$ peças deveriam ocorrer aproximadamente $n e^{-\lambda}$ peças sem defeitos. No entanto, na prática, às vezes acontece que o número 
de peças sem defeitos é muito maior do que seria esperado com base no número de defeitos que ocorrem nas peças imperfeitas. Uma interpretação plausível é que leves mudanças não observáveis nas condições que envolvem o processo fazem com que este oscile aleatoriamente entre o estado perfeito, em que a ocorrência de defeitos é extremamente rara, e o estado imperfeito, em que defeitos ocorrem, porém não necessariamente.

LAMBERT (1992) apresenta um exemplo em que dois tratamentos experimentais diferentes resultaram na mesma média para o número de defeitos observados em determinada peça e, no entanto, o estado perfeito do processo de fabricação foi mais provável em um dos tratamentos, enquanto que a média do número de defeitos que ocorrem no estado imperfeito foi menor no outro tratamento. Ou seja, este modelo pode mostrar, não apenas quais tratamentos resultam na menor média do número de ocorrências do evento estudado, mas também o modo como esses tratamentos determinam que suas médias sejam baixas. A autora mostra como ajustar um modelo Poisson inflacionado de zeros a dados de contagem, no qual ambos os parâmetros, $\lambda$ e $p$, dependem das covariáveis do modelo e, entre si, podem ser independentes ou estar relacionados através de uma função.

RIDout \& DemÉtrio (1994) propõem como alternativa para a análise dos dados referentes ao método da contracepção do anel vaginal, estudados por CAMPBEll et al. (1991), o modelo de regressão Poisson inflacionado de zeros, segundo o qual as mulheres seriam separadas em suscetíveis e não suscetíveis à expulsão do anel, ao invés de serem separadas com base na ocorrência, ou não, da primeira expulsão durante o período estudado, como acontece segundo o modelo Poisson truncado.

No caso de experimentos com cultura de tecidos em que a variável estudada é o número de calos produzidos por explante, por exemplo, pode-se admitir a distribuição Poisson inflacionada de zeros para a mesma, considerando-se que há dois tipos de explantes: os que regeneram, e os que não regeneram, sendo $p$ a probabilidade do explante regenerar e $\lambda$ a média do número de calos produzidos 
quando o explante regenera, número este que segue a distribuição Poisson $(\lambda)$.

\subsection{As distribuições Poisson compostas e a distribuição binomial negativa}

Uma outra forma de se abordar a falta de ajuste do modelo Poisson para dados que, a princípio, seriam bem explicados por este modelo é assumir que o fenômeno estudado ocorre segundo uma distribuição Poisson composta.

Um exemplo de distribuição Poisson composta é o caso em que a variável discreta $Y$ sob estudo consiste na soma de $N$ variáveis aleatórias $Z_{k}$ 's, $k=1,2, \ldots, N$, independentes e identicamente distribuídas, onde $N$ tem distribuição de Poisson e independe de $Z_{k}$, qualquer que seja $k$. Ou seja, cada evento $k$ contribui com uma quantidade aleatória $Z$ ao total $Y$ :

$$
Y=Z_{1}+Z_{2}+\cdots+Z_{N}
$$

É o que ocorre no exemplo apresentado por HINDE (1982), em que $Y_{i j}$ representa o número de migrantes da região $i$ para a região $j$ num estudo realizado na Grã-Bretanha e cujo principal objetivo foi associar o número de migrantes $Y_{i j}$ a algumas variáveis explanatórias, dentre as quais as populações $p_{i}$ e $p_{j}$ das regiões $i$ e $j$ respectivamente, e a distância $d_{i j}$ entre as regiões $i$ e $j$. Admite-se, a princípio, que o número de migrantes $Y_{i j}$ segue a distribuição de Poisson com média $\mu_{i j}$, e um modelo comumente usado em tais estudos para a esperança de $Y_{i j}$ é dado por:

$$
E\left(Y_{i j}\right)=\mu_{i j}=\frac{\alpha p_{i}^{\beta_{1}} p_{j}^{\beta_{2}}}{d_{i j}}
$$

onde $\alpha, \beta_{1}$, e $\beta_{2}$ são parâmetros desconhecidos. No entanto, quando se utiliza o modelo Poisson para a variável $Y_{i j}$, com média $\mu_{i j}$ dada pela equação (16), não se obtém um bom ajuste. Isto se deve ao fato de os indivíduos não constituírem unidades de migração independentes, as quais são mais razoavelmente constituŕdas por faḿlias. Então, o número total de migrantes da região $i$ para a região $j$ pode 
ser representado por:

$$
Y_{i j}=Z_{1}+Z_{2}+\cdots+Z_{N_{i j}}
$$

onde $N_{i j}$ é o número de famílias que migram da região $i$ para a região $j$, para o qual assume-se a distribuição de Poisson com média $\mu_{i j}$ dada pela equação (16), e $Z_{k}$ 's, $k=1,2, \ldots, N_{i j}$, são variáveis aleatórias independentes e identicamente distribuídas de acordo com a distribuição do tamanho da família na Grã-Bretanha. Aplicando-se este novo modelo obtém-se um ajuste muito melhor, o que indica ser mais razoável assumir a família como unidade de migração, e não o indivíduo.

Em geral, não se obtém um bom ajuste quando se admite o modelo Poisson para uma variável aleatória $Y$ com distribuição Poisson composta tal como a descrita pela equação (15), pois neste caso é comum ocorrer o fenômeno conhecido como superdispersão, em que a variância amostral dos dados é maior que a variância esperada segundo o modelo adotado. De fato, partindo-se de (15) tem-se:

$$
\begin{gathered}
E(Y)=E(N) E(Z) \\
V(Y)=E(N) E\left(Z^{2}\right)
\end{gathered}
$$

(MCCullagh \& Nelder, 1989), de modo que, se

$$
E\left(Z^{2}\right)>E(Z)
$$

então

$$
V(Y)>E(Y)
$$

e, neste caso, se for admitido o modelo Poisson para $Y$, segundo o qual

$$
V(Y)=E(Y)
$$

é de se esperar que o ajuste não seja bom, devido à ocorrência de superdispersão.

A superdispersão é um fenômeno que ocorre ocasionalmente nos modelos binomial e de Poisson e, quando está associada a este último modelo, é também chamada de variação extra-Poisson. A superdispersão reflete alguma fonte de variação dos dados que não está sendo explicada pelo modelo, podendo estar associada 
tanto a variáveis explanatórias importantes não consideradas, como à ocorrência de agrupamentos nos dados, gerados por observações correlacionadas. É o que ocorre no exemplo apresentado por HINDE (1982), já citado, em que existe correlação entre os indivíduos da mesma família, que migram da região $i$ para a região $j$.

Os dados apresentados por AITKIN et al. (1989) referentes ao número de faltas à escola dadas por crianças aborígenes também são um exemplo de dados de contagem que apresentam superdispersão quando explicados pelo modelo Poisson. Uma possível explicação para este fenômeno é que um grande motivo que leva as crianças a faltarem à escola, que são as doenças, tende a produzir agrupamentos de faltas, ou seja, se uma criança fica doente num dia e falta à escola, ela, mais provavelmente que outras crianças, faltará no próximo ou nos próximos dias. Se a superdispersão estiver relacionada a variáveis explanatórias, então o correto é tentar identificar as variáveis que explicam esse fenômeno. Se, no entanto, esta não for a causa real do problema, uma alternativa é admitir um modelo Poisson composto para os dados.

Uma maneira de se considerar a distribuição Poisson composta é admitir-se uma distribuição de Poisson com parâmetro $\lambda$, onde $\lambda$ não é constante, mas uma variável aleatória contínua. Para a variável produção de calos por explante, por exemplo, ao invés de se considerar que haja explantes que regeneram e outros que não regeneram, pode-se admitir, como alternativa para a falta de ajuste do modelo Poisson, que a regeneração ocorre de acordo com a distribuição de Poisson para todos os explantes, mas que a taxa $\lambda$ do processo varia de explante para explante como uma variável aleatória contínua. Neste caso, se for assumida a distribuição gama para $\lambda$, então o número total de calos produzidos por explante irá seguir a distribuição binomial negativa. Ou seja, pode ser verificado que, se $Y$ e $\lambda$ são duas variáveis aleatórias tais que

$$
Y \mid \lambda \sim \operatorname{Poisson}(\lambda) \text { e } \lambda \sim \Gamma(k, v)
$$


então $Y$ tem distribução binomial negativa, com função de probabilidade dada por:

$$
f(y ; \mu, k)=\frac{(y+k-1) !}{y !(k-1) !}\left(\frac{k}{\mu+k}\right)^{k}\left(\frac{\mu}{\mu+k}\right)^{y} I_{\{0,1,2, \ldots\}}(y),
$$

onde $k>0$ e $\mu=E(Y)>0$.

Além disso, tem-se que $Y$ e $\lambda$ têm a mesma esperança matemática, pois

$$
E(Y)=E[E(Y \mid \lambda)]
$$

(Mood et al., 1974), e, portanto,

$$
E(Y)=E(\lambda)=\mu
$$

Por outro lado, considerando-se que

$$
V(Y)=E[V(Y \mid \lambda)]+V[E(Y \mid \lambda)]
$$

(Mood et al., 1974), tem-se:

$$
\begin{aligned}
& V(Y)=E(\lambda)+V(\lambda) \\
& V(Y)=\mu+\frac{\mu^{2}}{k} \\
& V(Y)=\mu\left(1+\frac{\mu}{k}\right),
\end{aligned}
$$

o que demonstra que, ao se considerar a distribuição de Poisson para a variável aleatória $Y$, tem-se uma distribuição de Poisson com superdispersão, ou seja, cuja variância é maior que a média, pois, fazendo-se

$$
\left(1+\frac{\mu}{k}\right)=\phi
$$

a equação (20) fica:

$$
V(Y)=\phi \mu=\phi V(\mu)
$$

e, uma vez que $\mu>0$ e $k>0$, tem-se que $\phi>1$, o que expressa a superdispersão dos dados. Portanto, o modelo binomial negativo apresenta-se como uma alternativa para explicar esse tipo de resposta $Y$. 
Dependendo da distribuição que se admite para $\lambda$, obtêm-se outros modelos Poisson compostos. No entanto, uma razão para se considerar a distribuição gama é que esta implica numa distribuição conhecida para $Y$, a distribuição binomial negativa. Se o parâmetro $k$ desta distribuição é fixo e conhecido, então tem-se que a distribuição binomial negativa pertence à família exponencial, podendo-se adotar a teoria de modelos lineares generalizados no ajuste do modelo.

$\mathrm{Na}$ forma apresentada pela equação (1), a função de probabilidade da distribuição binomial negativa, dada pela expressão (18), fica:

$$
f\left(y_{i} ; \mu_{i}, k\right)=\exp \left\{\left[y_{i} \ln \left(\frac{\mu_{i}}{\mu_{i}+k}\right)-k \ln \left(\frac{\mu_{i}+k}{k}\right)\right]+\ln \left[\frac{\Gamma\left(y_{i}+k\right)}{y_{i} ! \Gamma(k)}\right]\right\}
$$

onde $\ln \left(\frac{\mu_{i}}{\mu_{i}+k}\right)=\theta_{i} ; k \ln \left(\frac{\mu_{i}+k}{k}\right)=b\left(\theta_{i}\right) ; \ln \left[\frac{\Gamma\left(y_{i}+k\right)}{y_{i} ! \Gamma(k)}\right]=c\left(y_{i} ; \phi\right) ; \phi=1 ;$ e $w_{i}=1$

Aplicando-se na equação (21) os resultados expressos pelas equaçôes (2) e (3), obtêm-se a esperança e a variância de $Y_{i}$, dadas por:

$$
\begin{aligned}
& E\left(Y_{i}\right)=b^{\prime}\left(\theta_{i}\right)=\mu_{i}, \quad \mathrm{e} \\
& V\left(Y_{i}\right)=b^{\prime \prime}\left(\theta_{i}\right)=V\left(\mu_{i}\right)=\frac{\mu_{i}}{k}\left(k+\mu_{i}\right) .
\end{aligned}
$$

\subsection{Teste do cociente variância-média para a distribuição dos dados}

Como afirma DiAz (1994), o teste do cociente variância-média é apropriado quando não se têm muitos elementos acerca dos dados e se necessita uma análise exploratória inicial das observações visando identificar sua distribuição.

Este teste é baseado na relação de igualdade entre a média e a variância da distribuição de Poisson.

São três as possíveis relações existentes entre a média, $\mu$, e a variância, $\sigma^{2}$, de uma população, ou seja,

(a) $\mu<\sigma^{2}$, 
(b) $\mu=\sigma^{2}$,

(c) $\mu>\sigma^{2}$.

Conhecidas distribuições de probabilidade consistem em modelos adequados para cada uma dessas situações. A distribuição de Poisson é um modelo adequado para a relação de igualdade entre os parâmetros $\mu$ e $\sigma^{2}$.

Dada uma amostra de uma população, o índice dado pelo cociente entre a variância amostral e a estimativa da variância da população obtida segundo a distribuição de Poisson deve ser próximo de 1 se as observações estiverem em concordância com esta distribuição. Ou seja, se a hipótese de que as observações seguem a distribuição de Poisson for verdadeira, então

$$
I=\frac{s^{2}}{\bar{y}}=\frac{\sum_{i=1}^{n}\left(y_{i}-\bar{y}\right)^{2}}{\bar{y}(n-1)} \approx 1
$$

onde $s^{2}$ é a variância amostral; $\bar{y}$ é a estimativa da variância da distribuição de Poisson; $y_{i}$ representa a observação $i, i=1,2, \ldots, n$; e $n$ é o tamanho da amostra.

Segundo ElliotT (1979), a expressão $I(n-1)$ é bem aproximada pela distribuição de $\chi^{2}$ com $(n-1)$ graus de liberdade, podendo-se escrever:

$$
I(n-1)=\frac{\sum_{i=1}^{n}\left(y_{i}-\bar{y}\right)^{2}}{\bar{y}}=\frac{s^{2}(n-1)}{\bar{y}} \sim \chi_{(n-1)}^{2} .
$$

Portanto, este teste aceita a hipótese $H_{0}$ de que os dados concordam com uma distribuição de Poisson, ao nível $(1-\alpha)$ de probabilidade, se

$$
\chi_{\left[1-\frac{\alpha}{2} ;(n-1)\right]}^{2}<I(n-1)<\chi_{\left[\frac{\alpha}{2} ;(n-1)\right]}^{2}
$$

onde $\alpha=P\left[X \geq \chi_{[\alpha ; v]}^{2}\right]$, sendo $X$ uma variável aleatória com distribuição de $\chi^{2}$ com $v$ graus de liberdade.

Para amostras grandes $(n>31)$ assume-se que $\sqrt{2 \chi^{2}}$ tem distribuição aproximadamente normal com média $\sqrt{2(n-1)-1}$ e variância unitária (ELLIOTT, 1979). Portanto, aceita-se a hipótese $H_{0}$ de que os dados seguem a distribuição de Poisson, ao nível $(1-\alpha)$ de probabilidade, se

$$
|d|=|\sqrt{2 I(n-1)}-\sqrt{2(n-1)-1}|<Z_{\frac{\alpha}{2}}
$$


onde $\frac{\alpha}{2}=P\left[X \geq Z_{\frac{\alpha}{2}}\right], P\left[0<X<Z_{\frac{\alpha}{2}}\right]=\frac{1-\alpha}{2}$, sendo $X$ uma variável aleatória com distribuição normal padrão.

A rejeição de $H_{0}$ pode se dar de duas formas:

(a) $I(n-1) \geq \chi_{\left[\frac{\alpha}{2} ;(n-1)\right]}^{2}$, ou, se $n>31, d \geq Z_{\frac{\alpha}{2}}$, o que indica, possivelmente, que $\mu>\sigma^{2}$,

(b) $I(n-1) \leq \chi_{\left[1-\frac{\alpha}{2} ;(n-1)\right]}^{2}$, ou, se $n>31, d \leq-Z_{\frac{\alpha}{2}}$, o que indica, possivelmente, que $\mu<\sigma^{2}$.

Portanto, este teste dá uma indicação do tipo de relação existente entre a média e a variância dos dados.

\subsection{Teste para superdispersão}

Nos modelos de regressão Poisson tem-se que variáveis observadas independentes $Y_{i}^{\prime}$ 's, $i=1,2, \ldots, n$, são explicadas por vetores de covariáveis $x_{i}$ 's, $x_{i}=\left(X_{1 i}, X_{2 i}, \ldots, X_{p_{i}}\right)$, sendo que a distribuição de $Y_{i}$ dado $x_{i}$ é Poisson com média $\mu_{i}=\mu_{i}\left(x_{i}, \beta\right)$, onde $\beta$ é um vetor $p \times 1$ de parâmetros desconhecidos, o que, por convenção, denota-se por $Y_{i} \sim$ Poisson $\left(\mu_{i}\right)$. (O modelo log-linear é o mais largamente utilizado modelo de regressão Poisson e, conforme foi referido no item 2.2, neste caso tem-se $\ln \mu_{i}=x_{i} \beta$ ).

Para considerar a possível ocorrência de superdispersão num modelo Poisson, este teste, introduzido por DEAN \& LAWLESS (1989), consiste em estender o que foi dito sobre a relação entre $Y_{i}$ e o vetor de covariáveis, $x_{i}$, a modelos de efeitos aleatórios. Ou seja, considerando-se as variáveis aleatórias positivas contínuas $\nu_{i}, \nu_{2}, \ldots, \nu_{n}$, independentes e identicamente distribuídas e tais que, dado $x_{i}$ e $\nu_{i}, Y_{i}$ tem distribuição Poisson $\left(\mu_{i} \nu_{i}\right)$; assumindo-se que as variáveis aleatórias $\nu_{i}$ 's têm os dois primeiros momentos finitos; e, sem perda de generalidade, tomando-se $E\left(\nu_{i}\right)=1$ e $V\left(\nu_{i}\right)=\tau ;$ então,

$$
V\left(Y_{i} \mid x_{i}\right)=\mu_{i}+\tau \mu_{i}^{2}
$$


de forma que se pode testar o modelo Poisson versus o modelo Poisson com superdispersão testando-se

$$
H_{0}: \tau=0 \quad \text { versus } \quad H_{1}: \tau>0
$$

A relação dada pela equação (23) está demonstrada em Collings \& Margolin (1985). Para o caso particular em que se tem

$$
k \nu_{i} \sim \Gamma(k, 1)
$$

para qualquer que seja $i$, onde $k$ é o parâmetro de escala da distribuição gama, então

$$
\begin{aligned}
& E\left(k \nu_{i}\right)=k \Rightarrow E\left(\nu_{i}\right)=1 \\
& V\left(k \nu_{i}\right)=k \Rightarrow V\left(\nu_{i}\right)=\frac{1}{k}=\tau
\end{aligned}
$$

e, escrevendo-se a expressão (17) na forma

$$
Y_{i} \mid \nu_{i} \sim \operatorname{Poisson}\left(\mu_{i} \nu_{i}\right) \text { e } k \nu_{i} \sim \Gamma(k, 1)
$$

chega-se à expresão (19), ou seja,

$$
V\left(Y_{i}\right)=\mu_{i}+\frac{\mu_{i}^{2}}{k}
$$

Mas, $1 / k=\tau$ e, portanto, fica demonstrada a equação (23) para o caso particular em que $k \nu_{i}$ tem distribuição $\Gamma(k, 1)$.

Este teste é uma extensão, para situações de regressão mais gerais, do teste proposto por Collings \& MARgolin (1985) para o modelo binomial negativo. A estatística do teste é dada por:

$$
T=\frac{1}{2} \sum_{i=1}^{n}\left[\left(Y_{i}-\hat{\mu}_{i}\right)^{2}-Y_{i}\right]
$$

onde $\hat{\mu}_{i}=\hat{\mu}_{i}\left(x_{i}, \hat{\beta}\right)$ e $\hat{\beta}$ é a estimativa de máxima verossimilhança obtida para $\beta$ sob o modelo Poisson.

Quando $n$, o tamanho da amostra, tende para infinito, e sob determinadas condições acerca dos vetores de variáveis explanatórias, $x_{i}$ 's, e dos parâmetros $\mu_{i}$ 's, as quais permitem a aplicação de máxima verossimilhança assintótica 
regular, tem-se que, sob $H_{0}: \tau=0, T /\left(\frac{1}{2} \sum_{i} \mu_{i}^{2}\right)^{1 / 2}$ converge em distribuição para a distribuição normal padrão. Portanto, uma estatística equivalente, estandartizada assintoticamente, é:

$$
T_{1}=\frac{\sum_{i=1}^{n}\left[\left(Y_{i}-\hat{\mu}_{i}\right)^{2}-Y_{i}\right]}{\left(2 \sum_{i=1}^{n} \hat{\mu}_{i}^{2}\right)^{1 / 2}} .
$$

Resultados de simulação indicam que, quando $n$ tende para infinito, a distribuição de $T_{1}$ aproxima-se lentamente da normal, portanto, a menos que $n$ seja muito grande, a aproximação $T_{1} \sim N(0,1)$ não é satisfatória.

Um ajustamento para $T_{1}$ que produz uma estatística com as mesmas propriedades assintóticas desta e, além disso, fornece melhores aproximações para o cálculo de valores críticos e níveis de significância é:

$$
T_{a}=\frac{\sum_{i=1}^{n}\left[\left(Y_{i}-\hat{\mu}_{i}\right)^{2}-Y_{i}+h_{i} \hat{\mu}_{i}\right]}{\left(2 \sum_{i=1}^{n} \hat{\mu}_{i}^{2}\right)^{1 / 2}}
$$

onde $h_{i}$ é o i-ésimo elemento da diagonal da matriz $H$ de ordem $n$, tal que $H=\hat{W}^{1 / 2} X\left(X^{\prime} \hat{W} X\right)^{-1} X^{\prime} \hat{W}^{1 / 2}$, sendo $X$ a matriz $n \times p$ do modelo e $\hat{W}$ uma matriz $n \times n$ de pesos tal que

$$
\hat{W}=\operatorname{diag}\left\{\left.\frac{w_{i}}{V\left(\hat{\mu}_{i}\right)}\left(\frac{\mathrm{d} g^{-1}\left(\eta_{i}\right)}{\mathrm{d} \eta_{i}}\right)^{2}\right|_{\eta_{i}=\hat{\eta}_{i}}\right\}
$$

A estatística $T_{a}$ converge para a normalidade mais rapidamente que a estatística $T_{1}$, de forma que, utilizando-se a estatística $T_{a}$, aceita-se $H_{0}: \tau=0$ ao nível $(1-\alpha)$ de probabilidade se

$$
T_{a}<Z_{\alpha}
$$

onde $\alpha=P\left[X \geq Z_{\alpha}\right]$, sendo $X$ uma variável aleatória com distribuição normal padrão. 


\section{MATERIAL E MÉTODOS}

\subsection{Material}

Neste trabalho são utilizados três conjuntos de dados, os quais são descritos e apresentados a seguir. Os Conjuntos A e B foram fornecidos pelo Departamento de Genética da ESALQ/USP e o Conjunto C pelo Centro de Energia Nuclear na Agricultura (CENA).

Conjunto A Os dados do Quadro 1 referem-se ao número de calos produzidos por explante de Stylosanthes scabra, espécie leguminosa usada como forrageira, mantido em meio de cultura por 28 dias. São resultantes de um experimento fatorial $3^{2}$ instalado no delineamento inteiramente casualizado, em que os fatores estudados foram o ácido naftaleno-acético (ANA) no meio de cultura e o tipo de radiação luminosa a que a cultura fica exposta. Os níveis estudados para ANA foram 0,$0 ; 0,5$ e 1,0 mg/l, e para o tipo de radiação luminosa foram lâmpada branca fria, lâmpada de amplo espectro e escuro. A unidade experimental, ou parcela, constituiu-se de um explante, e cada explante foi cultivado em um tubo de ensaio. O objetivo do experimento foi:

1. verificar a existência de efeito de interação entre os fatores estudados, dentro dos níveis fixados, sobre o número de calos produzidos por explante;

2. no caso de existência do efeito de interação entre os fatores, estudar este efeito, e, no caso de não existência, estudar o efeito principal de cada fator sobre o número de calos produzidos por explante. 
Conjunto B Os dados do Quadro 2 referem-se ao número de calos produzidos por explante de Stylosanthes scabra mantido em meio de cultura por 28 dias. São resultantes de um experimento inteiramente casualizado com 14 tratamentos constituídos por: testemunha; água de coco no meio de cultura nas concentrações 5\%, 10\% e 15\%; e TIBA (anti-auxina) no meio de cultura nas concentrações 0,$05 ; 0,10$ e $0,20 \mathrm{mg} / 1$; todos combinados com ausência e presença de carvão ativado no meio de cultura. A unidade experimental, ou parcela, constituiu-se de um explante, e cada explante foi cultivado em um tubo de ensaio. $\mathrm{O}$ objetivo do experimento foi:

1. verificar a existência de efeito de interação entre presença de carvão, presença de água de coco e presença de TIBA no meio de cultura, nos níveis fixados para essas substâncias, sobre o número de calos produzidos por explante;

2. no caso de existência de efeito de interação, estudar este efeito, e, no caso de não existência, estudar o efeito principal das substâncias estudadas sobre o número de calos produzidos por explante.

Quadro 1: Número de calos produzidos por explante de Stylosanthes scabra após 28 dias em meio de cultura.

\begin{tabular}{|c|c||ccccccccccccccc|}
\hline \multirow{2}{*}{$\begin{array}{c}\text { Tipo de rad. } \\
\text { luminosa }\end{array}$} & \multicolumn{1}{|c||}{$\begin{array}{c}\text { Dose de } \\
\text { ANA (mg/l) }\end{array}$} & \multicolumn{110}{|c|}{ 1 } & 2 & 3 & 4 & 5 & 6 & 7 & 8 & 9 & 10 & 11 & 12 & 13 & 14 & 15 \\
\hline \hline \multirow{3}{*}{ Lâmpada } & 0,0 & 8 & 13 & 12 & 8 & 13 & 12 & 10 & 9 & 12 & 10 & 6 & 7 & 10 & 11 & \\
branca fria & 0,5 & 0 & 2 & 0 & 1 & 0 & 2 & 0 & 1 & 0 & 1 & 0 & 2 & 3 & 0 & \\
& 1,0 & 0 & 0 & 0 & 0 & 0 & 0 & 1 & 0 & 1 & 0 & 0 & 0 & 0 & 0 & \\
\hline Lâmpada & 0,0 & 6 & 8 & 7 & 8 & 10 & 11 & 10 & 12 & 10 & 11 & 6 & 13 & 6 & 8 & 9 \\
de amplo & 0,5 & 0 & 2 & 3 & 0 & 0 & 0 & 0 & 0 & 0 & 0 & 0 & 0 & 0 & 0 & \\
espectro & 1,0 & 2 & 0 & 0 & 0 & 0 & 0 & 0 & 0 & 0 & 0 & 0 & 0 & 0 & 0 \\
\hline & 0,0 & 0 & 0 & 0 & 1 & 0 & 0 & 1 & 0 & 2 & 0 & 0 & 0 & 0 & 0 \\
Escuro & 0,5 & 0 & 0 & 0 & 0 & 0 & 0 & 0 & 0 & 0 & 0 & 0 & 0 & 0 & 0 \\
& 1,0 & 0 & 0 & 0 & 0 & 0 & 0 & 0 & 0 & 0 & 0 & 0 & 0 & 0 & 0 \\
\hline
\end{tabular}


Quadro 2: Número de calos produzidos por explante de Stylosanthes scabra após 28 dias em meio de cultura.

\begin{tabular}{|c|c|c|c|c|c|c|c|c|c|c|c|}
\hline \multirow{3}{*}{$\begin{array}{l}\text { Carvão } \\
\text { ativado }\end{array}$} & \multirow{3}{*}{ Suplemento } & \multicolumn{10}{|c|}{ Repetição } \\
\hline & & 1 & 2 & 3 & 4 & 5 & 6 & 7 & 8 & 9 & 10 \\
\hline & & 11 & 12 & 13 & 14 & 15 & 16 & 17 & 18 & 19 & 20 \\
\hline \multirow{14}{*}{ Ausente } & \multirow{2}{*}{ Testemunha } & 10 & 6 & 10 & 2 & 5 & 5 & 3 & 4 & 8 & 2 \\
\hline & & 2 & 2 & 2 & 4 & 2 & 1 & 11 & 2 & 3 & 2 \\
\hline & \multirow{2}{*}{$5 \%$ de água de coco } & 50 & 30 & 35 & 20 & 15 & 16 & 35 & 25 & 10 & 10 \\
\hline & & 15 & 10 & 25 & 30 & 30 & 20 & 30 & 20 & 20 & 32 \\
\hline & \multirow{2}{*}{$10 \%$ de água de coco } & 80 & 80 & 30 & 45 & 10 & 10 & 80 & 10 & 60 & 65 \\
\hline & & 45 & 29 & 15 & 10 & 10 & 16 & 70 & 10 & 40 & 80 \\
\hline & \multirow{2}{*}{$15 \%$ de água de coco } & 70 & 72 & 10 & 10 & 60 & 40 & 39 & 20 & 50 & 25 \\
\hline & & 14 & 10 & 45 & 10 & 50 & 10 & 60 & 20 & 35 & 60 \\
\hline & \multirow{2}{*}{$0,05 \mathrm{mg} / \mathrm{l} \mathrm{de}$ TIBA } & 22 & 12 & 30 & 40 & 2 & 2 & 1 & 13 & 18 & 3 \\
\hline & & 20 & 2 & 14 & 35 & 7 & 1 & 1 & 1 & 1 & 10 \\
\hline & \multirow{2}{*}{$0,10 \mathrm{mg} / \mathrm{l}$ de TIBA } & 18 & 15 & 15 & 30 & 7 & 3 & 12 & 20 & 18 & 18 \\
\hline & & 2 & 10 & 6 & 25 & 1 & 4 & 2 & 45 & 40 & 2 \\
\hline & \multirow{2}{*}{$0,20 \mathrm{mg} / \mathrm{l} \mathrm{de}$ TIBA } & 20 & 12 & 15 & 3 & 32 & 1 & 19 & 8 & 1 & 1 \\
\hline & & 1 & 23 & 50 & 20 & 30 & 15 & 20 & 7 & 13 & 50 \\
\hline \multirow{14}{*}{$\begin{array}{c}\text { Presente } \\
(5 \mathrm{~g} / \mathrm{l})\end{array}$} & \multirow{2}{*}{ Testemunha } & 4 & 0 & 1 & 0 & 0 & 2 & 2 & 0 & 0 & 2 \\
\hline & & 0 & 0 & 0 & 1 & 0 & 0 & 4 & 1 & 0 & 1 \\
\hline & \multirow[b]{2}{*}{$5 \%$ de água de coco } & 0 & 0 & 1 & 0 & 1 & 0 & 0 & 4 & 0 & 3 \\
\hline & & 0 & 0 & 3 & 0 & 0 & 0 & 0 & 2 & 2 & 2 \\
\hline & \multirow{2}{*}{$10 \%$ de água de coco } & 1 & 0 & 0 & 0 & 0 & 0 & 3 & 0 & 1 & 0 \\
\hline & & 0 & 0 & 0 & 0 & 0 & 0 & 0 & 0 & 1 & 3 \\
\hline & \multirow[b]{2}{*}{$15 \%$ de água de coco } & 0 & 0 & 0 & 0 & 1 & 0 & 0 & 0 & 0 & 0 \\
\hline & & 0 & 0 & 0 & 0 & 0 & 0 & 0 & 1 & 0 & 2 \\
\hline & \multirow{2}{*}{$0,05 \mathrm{mg} / 1 \mathrm{de}$ TIBA } & 0 & 1 & 0 & 0 & 0 & 0 & 1 & 0 & 0 & 0 \\
\hline & & 0 & 0 & 0 & 0 & 0 & 0 & 0 & 0 & 1 & 0 \\
\hline & \multirow{2}{*}{$0,10 \mathrm{mg} / \mathrm{l} \mathrm{de}$ TIBA } & 0 & 0 & 1 & 2 & 1 & 1 & 0 & 0 & 0 & 0 \\
\hline & & 0 & 0 & 0 & 0 & 0 & 0 & 0 & 0 & 5 & 6 \\
\hline & \multirow{2}{*}{$0,20 \mathrm{mg} / \mathrm{l}$ de TIBA } & 0 & 0 & 0 & 1 & 2 & 2 & 0 & 0 & 0 & 0 \\
\hline & & 0 & 0 & 0 & 0 & 0 & 0 & 1 & 0 & 2 & 0 \\
\hline
\end{tabular}


Conjunto $\mathrm{C}$ Os dados do Quadro 3 referem-se ao número de calos produzidos por explante. São resultantes de um experimento fatorial $3 \times 4$ instalado no delineamento inteiramente casualizado, com número variável de repetições por tratamento, e 10 sub-amostras por repetição. Cada repetição constituiu-se de uma placa-de-Petri com 10 explantes, cada um destes constituindo uma subamostra. Os fatores estudados foram: o regulador de crescimento Picloran no meio de cultura, nos níveis de 4,$5 ; 7,5$ e $10,0 \mathrm{mg} / \mathrm{l}$, e a espécie doadora do explante, em quatro níveis, todos constituídos por espécies pertencentes ao gênero Passiflora (maracujá), a saber, P. quadrangularis, P. incarnata, $P$. edulis e $P$. maliformis. $\mathrm{O}$ objetivo do experimento foi:

1. verificar a existência de efeito de interação entre os fatores estudados, dentro dos níveis fixados, sobre o número de calos produzidos por explante;

2. no caso de existência do efeito de interação entre os fatores, estudar este efeito, e, no caso de não existência, estudar o efeito principal do fator regulador de crescimento sobre o número de calos produzidos por explante. 
Quadro 3: Número de calos produzidos por explante.

\begin{tabular}{|c|c|c|c|c|c|c|c|c|c|c|c|c|}
\hline \multirow{2}{*}{ Espécie } & \multirow{2}{*}{$\begin{array}{c}\text { Dose de Picloran } \\
(\mathrm{mg} / \mathrm{l})\end{array}$} & \multirow{2}{*}{ Repetição } & \multicolumn{10}{|c|}{ Sub-amostra } \\
\hline & & & 1 & 2 & 3 & 4 & 5 & 6 & 7 & 8 & 9 & 10 \\
\hline \multirow{13}{*}{$A^{\dagger}$} & \multirow{5}{*}{10,0} & 1 & 1 & 2 & 2 & 1 & 3 & 3 & 2 & 2 & 3 & 2 \\
\hline & & 2 & 4 & 1 & 4 & 3 & 3 & 4 & 3 & 2 & 2 & 5 \\
\hline & & 3 & 3 & 3 & 2 & 1 & 4 & 4 & 5 & 5 & 5 & 4 \\
\hline & & 4 & 4 & 4 & 3 & 4 & 3 & 4 & 4 & 6 & 5 & 4 \\
\hline & & 5 & 4 & 3 & 1 & 2 & 4 & 3 & 4 & 4 & 3 & 3 \\
\hline & \multirow{4}{*}{7,5} & 1 & 5 & 2 & 6 & 1 & 4 & 6 & 2 & 6 & 6 & 5 \\
\hline & & 2 & 1 & 4 & 4 & 2 & 4 & 3 & 2 & 3 & 3 & 2 \\
\hline & & 3 & 2 & 3 & 1 & 1 & 1 & 1 & 2 & 2 & 1 & 2 \\
\hline & & 4 & 2 & 4 & 3 & 3 & 2 & 2 & 2 & 1 & 1 & 2 \\
\hline & \multirow{4}{*}{4,5} & 1 & 2 & 4 & 0 & 4 & 2 & 2 & 0 & 0 & 1 & 4 \\
\hline & & 2 & 2 & 1 & 2 & 3 & 3 & 5 & 4 & 1 & 3 & 2 \\
\hline & & 3 & 3 & 4 & 2 & 2 & 2 & 2 & 2 & 0 & 2 & 1 \\
\hline & & 4 & 2 & 3 & 2 & 2 & 2 & 1 & 2 & 2 & 3 & 1 \\
\hline \multirow{10}{*}{$\mathrm{B}^{\ddagger}$} & \multirow{4}{*}{10,0} & 1 & 0 & 0 & 1 & 2 & 0 & 0 & 3 & 5 & 6 & 1 \\
\hline & & 2 & 0 & 1 & 0 & 0 & 1 & 0 & 2 & 1 & 0 & 0 \\
\hline & & 3 & 0 & 0 & 0 & 0 & 0 & 0 & 0 & 0 & 0 & 0 \\
\hline & & 4 & 0 & 2 & 0 & 0 & 0 & 0 & 0 & 0 & 0 & 0 \\
\hline & \multirow{4}{*}{7,5} & 1 & 4 & 0 & 0 & 0 & 0 & 0 & 0 & 0 & 0 & 0 \\
\hline & & 2 & 0 & 0 & 0 & 0 & 0 . & 0 & 0 & 0 & 0 & 0 \\
\hline & & 3 & 0 & 0 & 0 & 0 & 0 & 0 & 0 & 0 & 0 & 0 \\
\hline & & 4 & 2 & 1 & 0 & 0 & 0 & 0 & 0 & 0 & 0 & 0 \\
\hline & \multirow{2}{*}{4,5} & 1 & 6 & 0 & 0 & 0 & 0 & 0 & 0 & 0 & 0 & 0 \\
\hline & & 2 & 0 & 0 & 0 & 0 & 0 & 0 & 0 & 0 & 0 & 0 \\
\hline
\end{tabular}

... continua

\footnotetext{
${ }^{\dagger} \mathrm{A}=$ Passiflora quadrangularis

${ }^{\ddagger} \mathrm{B}=$ Passiflora incarnata
} 
Quadro 3: Número de calos produzidos por explante. (continuação)

\begin{tabular}{|c|c|c|c|c|c|c|c|c|c|c|c|c|}
\hline \multirow{2}{*}{ Espécie } & \multirow{2}{*}{$\begin{array}{l}\text { Dose de Picloran } \\
\qquad(\mathrm{mg} / \mathrm{l})\end{array}$} & \multirow{2}{*}{ Repetição } & \multicolumn{10}{|c|}{ Sub-amostra } \\
\hline & & & 1 & 2 & 3 & 4 & 5 & 6 & 7 & 8 & 9 & 10 \\
\hline \multirow{9}{*}{$\mathrm{C}^{\dagger \dagger}$} & \multirow{4}{*}{10,0} & 1 & 2 & 0 & 2 & 1 & 2 & 1 & 5 & 1 & 0 & 2 \\
\hline & & 2 & 4 & 1 & 2 & 1 & 0 & 0 & 3 & 2 & 3 & 3 \\
\hline & & 3 & 1 & 2 & 1 & 6 & 5 & 0 & 3 & 0 & 0 & 0 \\
\hline & & 4 & 9 & 7 & 4 & 10 & 6 & 4 & 7 & 7 & 7 & 4 \\
\hline & \multirow{4}{*}{7,5} & 1 & 8 & 7 & 8 & 4 & 8 & 6 & 8 & 4 & 6 & 9 \\
\hline & & 2 & 2 & 4 & 8 & 5 & 9 & 10 & 12 & 8 & 5 & 9 \\
\hline & & 3 & 0 & 0 & 0 & 0 & 0 & 0 & 0 & 0 & 0 & 0 \\
\hline & & 4 & 2 & 1 & 0 & 0 & 0 & 0 & 0 & 0 & 0 & 0 \\
\hline & 4,5 & 1 & 8 & 7 & 6 & 7 & 4 & 1 & 4 & 5 & 9 & 4 \\
\hline \multirow{12}{*}{$D^{\ddagger \ddagger}$} & \multirow{4}{*}{10,0} & 1 & 0 & 0 & 0 & 0 & 0 & 0 & 0 & 0 & 0 & 0 \\
\hline & & 2 & 3 & 2 & 1 & 1 & 0 & 0 & 0 & 0 & 0 & 0 \\
\hline & & 3 & 0 & 0 & 0 & 0 & 0 & 0 & 0 & 0 & 0 & 0 \\
\hline & & 4 & 0 & 0 & 0 & 0 & 0 & 0 & 0 & 0 & 0 & 0 \\
\hline & \multirow{4}{*}{7,5} & 1 & 0 & 0 & 0 & 0 & 0 & 0 & 0 & 0 & 0 & 0 \\
\hline & & 2 & 0 & 0 & 0 & 0 & 0 & 0 & 0 & 0 & 0 & 0 \\
\hline & & 3 & 0 & 0 & 0 & 0 & 0 & 0 & 0 & 0 & 0 & 0 \\
\hline & & 4 & 0 & 0 & 0 & 0 & 0 & 0 & 0 & 0 & 0 & 0 \\
\hline & \multirow{4}{*}{4,5} & 1 & 2 & 2 & 1 & 2 & 3 & 0 & 1 & 0 & 0 & 2 \\
\hline & & 2 & 2 & 2 & 0 & 3 & 2 & 0 & 0 & 0 & 2 & 1 \\
\hline & & 3 & 0 & 3 & 2 & 6 & 4 & 0 & 0 & 0 & 2 & 4 \\
\hline & & 4 & 1 & 0 & 0 & 0 & 0 & 0 & 0 & 0 & 0 & 0 \\
\hline
\end{tabular}

\footnotetext{
${ }^{{ }^{\dagger} \mathrm{C}}=$ Passiflora edulis

$\ddagger^{\ddagger} \mathrm{D}=$ Passiflora maliformis
} 


\subsection{Métodos}

\subsubsection{Testes preliminares}

Antes de se efetuar o ajuste dos modelos descritos no item 3.2.2, a seguir, os três conjuntos de dados utilizados neste trabalho foram submetidos ao teste do cociente variância-média e ao teste para detectar superdispersão, expostos nos ftens 2.6 e 2.7, respectivamente. Para cada conjunto de dados, o primeiro teste foi aplicado em cada um dos tratamentos, o que foi realizado no SAS ("Statistical Analysis System") (SAS INSTITUTE INC., 1988), de acordo com o programa apresentado no Apêndice 1, usando o Conjunto A de dados como exemplo. O teste para superdispersão foi realizado no GLIM ("Generalized Linear Interactive Modelling System") (PAYNE, 1986), e o programa utilizado, usando o Conjunto A de dados como exemplo, apresenta-se no Apêndice 2.

A utilização desses testes teve por objetivo a obtenção de resultados que assegurassem as conclusões obtidas com base nos resultados do ajuste dos modelos.

\subsubsection{Modelos}

\subsubsection{Modelo Poisson}

Uma vez que se trata de dados de contagem, admitiu-se, primeiramente, o modelo Poisson para explicar os dados apresentados no item anterior. Adotando-se a teoria de modelos lineares generalizados, o ajuste deste modelo foi feito no GLIM (PAYNE, 1986) partindo-se de:

$$
\begin{gathered}
E(Y)=\mu \\
\theta=\ln (\mu)=g(\mu)=\eta \\
\eta=X \beta
\end{gathered}
$$

onde $Y$ é o vetor $n \times 1$ das observações, tal que se admite $Y \sim \operatorname{Poisson}(\mu)$;

$\theta$ é o vetor do parâmetro natural ou canônico da distribuição de Poisson, de 
dimensão $n \times 1$;

$\eta$ é o vetor $n \times 1$ dos preditores lineares;

$X$ é a matriz $n \times p$ do delineamento; e

$\beta$ é o vetor $p \times 1$ de parâmetros desconhecidos do preditor linear do modelo.

O preditor linear, $\eta$, inicialmente adotado para cada conjunto de dados, de acordo com a estrutura dos experimentos que os geraram, descrita no item 3.1, foi o seguinte:

\section{Conjunto A}

$$
\begin{gathered}
\eta=\left(\eta_{i j k}\right) \\
\eta_{i j k}=m+l_{i}+d_{j}+l d_{i j}
\end{gathered}
$$

onde $\eta_{i j k}$ é o preditor linear correspondente à observação $y_{i j k}, k=1,2, \ldots, K_{i j}$ representando a k-ésima repetição do tratamento $T_{i j}$;

$m$ é uma constante inerente a todos os preditores lineares;

$l_{i}$ é o efeito do nłvel $i$ do fator tipo de radiação luminosa, $i=1,2,3$, sendo 1, 2 e 3 correspondentes a lâmpada branca fria, lâmpada de amplo espectro e escuro, respectivamente;

$d_{j}$ é o efeito da dose $j$ do fator ácido naftaleno-acético (ANA), $j=1,2,3$, sendo 1,2 e 3 correspondentes a 0,$0 ; 0,5$ e 1,0 $\mathrm{mg} / \mathrm{l}$, respectivamente; $l d_{i j}$ é o efeito da interação do nível $i$ do fator tipo de radiação luminosa com a dose $j$ do fator ANA.

\section{Conjunto B}

$$
\begin{gathered}
\eta=\left(\eta_{i j k}\right) \\
\eta_{i j k}=m+c_{i}+s_{j}+c s_{i j}
\end{gathered}
$$

onde $\eta_{i j k}$ é o preditor linear correspondente à observação $y_{i j k}, k=1,2, \ldots, 20$ representando as repetições do tratamento $T_{i j}$; $m$ é uma constante inerente a todos os preditores lineares; $c_{i}$ é o efeito do nível $i$ do fator carvão, $i=1,2$, onde 1 e 2 correspondem 
a ausência e presença de carvão ativado, respectivamente;

$s_{j}$ é o efeito do nível $j$ do fator suplemento, $j=1,2, \ldots, 7$, onde 1 corresponde à testemunha, 2,3 e 4 correspondem a água de coco nas concentrações de $5 \%, 10 \%$ e 15\%, respectivamente, e 5, 6 e 7 correspondem a TIBA nas concentrações de 0,$05 ; 0,10$ e $0,20 \mathrm{mg} / \mathrm{l}$, respectivamente; $c s_{i j}$ é o efeito da interação do nível $i$ do fator carvão com o nível $j$ do fator suplemento.

Conjunto $\mathrm{C}$

$$
\begin{gathered}
\eta=\left(\eta_{i j k l}\right) \\
\eta_{i j k l}=m+e_{i}+d_{j}+e d_{i j}+p_{k(i j)},
\end{gathered}
$$

onde $\eta_{i j k l}$ é o preditor linear correspondente à observação $y_{i j k l}, k=$ $1,2, \ldots, K_{i j}$ representando a k-ésima placa ou repetição do tratamento $T_{i j}$ e $l=1,2, \ldots, 10$ representando a l-ésima sub-amostra da placa ou repetição $k$;

$m$ é uma constante inerente a todos os preditores lineares;

$e_{i}$ é o efeito do nível $i$ do fator espécie, $i=1,2,3,4$, onde $1,2,3$ e 4 correspondem a $P$. quadrangularis, $P$. incarnata, $P$. edulis e P. maliformis, respectivamente;

$d_{j}$ é o efeito da dose $j$ do fator Picloran, $j=1,2,3$, onde 1,2 e 3 correspondem a 10,$0 ; 7,5$ e 4,5 $\mathrm{mg} / \mathrm{l}$, respectivamente;

$e d_{i j}$ é o efeito da interação do nível $i$ do fator espécie com a dose $j$ do fator Picloran;

$p_{k(i j)}$ é o efeito da placa $k$ do tratamento $T_{i j}, k=1,2, \ldots, K_{i j}$.

A macro utilizada para efetuar o ajuste deste modelo está contida no programa que consta do Apêndice 3, referente ao ajuste ao Conjunto A de dados.

\subsubsection{Modelo Poisson truncado}

Quando o modelo Poisson não ofereceu um bom ajuste, considerou- 
se, alternativamente, o modelo Poisson truncado para os dados. Partindo-se do exposto no item 2.3, o ajuste foi efetuado no GLIM através do modelo do usuário considerando-se a função de ligação

$$
\eta=g_{2}(\mu)=\ln (\mu-1)
$$

sugerida por Ridout \& DEMÉTRIo (1992), e adotando-se para a função $h(\mu)$, que estima o parâmetro $\lambda$, a aproximação

$$
h^{(1)}(\mu)=\frac{\mu\left\{1-\exp \left[-h^{(0)}(\mu)\right]\right\}^{2}-\left[h^{(0)}(\mu)\right]^{2} \exp \left[-h^{(0)}(\mu)\right]}{1-\left[h^{(0)}(\mu)+1\right] \exp \left[-h^{(0)}(\mu)\right]},
$$

obtida pelo método exposto no item 2.3 .

0 preditor linear, $\eta$, adotado neste modelo para cada conjunto de dados, salvo pela alteração nos valores assumidos pelos índices $j, k, \mathrm{e} l$ em virtude da exclusão das observações iguais a zero, foi o mesmo adotado no modelo Poisson. A macro utilizada para o ajuste desse modelo, apresentada por RIDout \& DEMÉtrio (1992), está incluída no programa referente ao ajuste ao Conjunto A de dados, que consta do Apêndice 4.

\subsubsection{Modelo binomial negativo}

Quando não se obteve um bom ajuste para os modelos Poisson e Poisson truncado, considerou-se o modelo binomial negativo para os dados, considerando-se fixo o parâmetro $k$ da distribuição. Ou seja, uma vez obtida uma estimativa para $k$, esta foi substituf́da na função de verossimilhança da distribuição para efetuar o ajuste do modelo. O ajuste foi efetuado no GLIM através do modelo do usuário, considerando-se a função de ligação canônica, ou seja, $\eta=\theta$, dada por:

$$
\eta=\ln \left(\frac{\mu}{\mu+k}\right) \Rightarrow \mu=k \frac{e^{\eta}}{1-e^{\eta}} .
$$

O preditor linear, $\eta$, adotado neste modelo, para cada conjunto de dados, é o mesmo adotado para o modelo Poisson, já descrito. A macro utilizada para efetuar o ajuste deste modelo está incluída no programa referente ao ajuste ao Conjunto $\mathrm{B}$ de dados, que se apresenta no Apêndice 7. 
Como estimativa para o parâmetro $k$, desconhecido, calculou-se a "deviance" residual do modelo para diversos valores de $k \mathrm{e}$ tomou-se aquele que forneceu o valor da "deviance" residual mais próximo do número de graus de liberdade a ela associado. Como será exposto adiante, é comum testar-se o ajuste de um modelo linear generalizado comparando-se sua "deviance" residual, para a qual se admite a distrubuição de $\chi^{2}$, com o valor crítico $\chi_{[\alpha ;(n-p)]}^{2}$, onde $\alpha$ é o nível de significância adotado, $n$ é o número de observações e $p$ é o número de parâmetros desconhecidos e independentes do modelo. Sabe-se que, se $V$ é uma variável aleatória com distribuição de $\chi_{(n-p)}^{2}$, então

$$
E(V)=n-p,
$$

e daf se considerar como estimativa para $k$ o valor do parâmetro que resulta na "deviance" residual do modelo mais próxima do número de graus de liberdade a ela associado. O programa em GLIM utilizado para a obtenção de tal valor de $k$ apresenta-se no Apêndice 8.

\subsubsection{Técnicas para verificação do ajuste do modelo}

Para verificação da qualidade do ajuste dos modelos, considerou-se a análise de "deviance", a análise de resíduos e algumas técnicas de diagnóstico para modelos lineares generalizados, como o gráfico normal de probabilidade para os valores absolutos dos componentes de "deviance" padronizados ("half normal plot") com envelope simulado, gráfico de índice para os componentes de "deviance" padronizados e para os componentes de "deviance" estudentizados externamente, e gráfico dos componentes de "deviance" padronizados versus tratamentos ordenados pelas médias.

\subsubsection{Análise de "deviance"}

A "deviance" é uma estatística proposta por NELDER \& WEDDERBURN (1972) para medir o ajuste de um modelo. É definida como duas vezes a diferença entre o máximo do logaritmo da função de verossimilhança do modelo 
saturado $\left(\hat{l}_{n}\right)$ e o máximo do logaritmo da função de verossimilhança do modelo corrente $\left(\hat{l}_{p}\right)$, onde modelo saturado é aquele em que se têm $n$ parâmetros, um para cada observação, e modelo corrente é aquele sob pesquisa, com $p$ parâmetros. Ou seja, adotando-se a notação usada por MCCullaGH \& Nelder (1989) para as distribuições da família exponencial dada pela equação (1), tem-se que a "deviance" é dada por:

$$
S_{p}=2\left(\hat{l}_{n}-\hat{l}_{p}\right)=\frac{1}{\phi} \sum_{i=1}^{n} 2 w_{i}\left\{y_{i}\left[\tilde{\theta}_{i}-\hat{\theta}_{i}\right]-b\left(\tilde{\theta}_{i}\right)+b\left(\hat{\theta}_{i}\right)\right\}=\frac{1}{\phi} \sum_{i=1}^{n} d_{i}^{2}=\frac{1}{\phi} D_{p},
$$

onde $S_{p}$ é chamada de "scaled deviance"; $\tilde{\theta}_{i}=\tilde{\theta}\left(y_{i}\right)$ e $\hat{\theta}_{i}=\hat{\theta}_{i}\left(\hat{\mu}_{i}\right)$ são as estimativas do parâmetro canônico sob os modelos saturado e corrente, respectivamente; e $D_{p}$ é chamada de "deviance".

A "scaled deviance" é a expressão da quantidade $D_{p}$ como um múltiplo do parâmetro de dispersão ou parâmetro de escala, $\phi$. Neste trabalho, o termo "deviance" será empregado, deste ponto em diante, para referir-se à quantidade $S_{p}$.

Cada valor $d_{i}^{2}$, na equação (25), é uma medida da distância entre o valor obsevado $y_{i}$ e seu correspondente valor ajustado $\hat{\mu}_{i}$, pois mede a diferença entre os logaritmos das funções de verossimilhança observada e ajustada para a observação de índice $i$. Dessa forma, a soma de todos os valores $d_{i}^{2}$ 's, $D_{p}$, mede a discrepância total entre as duas funções de verossimilhança, ou, equivalentemente, entre os modelos saturado e corrente. A medida que se incluem variáveis explanatórias no modelo, a "deviance" decresce até tornar-se nula para o modelo saturado. Quanto a sua distribuição de probabilidade, em algumas circunstâncias a "deviance" pode ser aproximada pela distribuição de $\chi^{2}$, como, por exemplo, em problemas que envolvem dados discretos e em que a amostra é grande. Em geral, no entanto, não se tem uma boa aproximação para a "deviance"pela distribuição de $\chi^{2}$, nem mesmo assintoticamente. Por outro lado, sabe-se que, dados dois modelos encaixados com $q$ e $p$ parâmetros, $q<p$, então a diferença

$$
S_{q}-S_{p}=\frac{1}{\phi}\left(D_{q}-D_{p}\right)
$$


assintoticamente tem distribuição de $\chi^{2} \operatorname{com}(p-q)$ graus de liberdade.

Embora na maioria dos casos a "deviance", $S_{p}$, não tenha distribuição de probabilidade conhecida, na prática usa-se testar o ajuste de um modelo comparando-se sua "deviance" residual com o valor crítico $\chi_{[\alpha ;(n-p)]}^{2}$, onde $\alpha$ é o nível de significância adotado, $n$ é o número de observações e $p$ é o número de parâmetros desconhecidos e independentes do modelo sob pesquisa. Se o ajuste for bom, a "deviance" residual deve ser menor do que ou igual a $\chi_{[\alpha ;(n-p)]}^{2}$. Da mesma forma, usa-se, também, a aproximação

$$
\frac{(n-p)\left(S_{q}-S_{p}\right)}{(p-q) S_{p}} \sim F_{[(p-q),(n-p)]}
$$

para testar o efeito dos termos que estão inclúdos no modelo com $p$ parâmetros e não estão incluídos no modelo com $q$ parâmetros.

\subsubsection{Análise de resíduos e diagnósticos}

As técnicas usadas para análise de resíduos e diagnósticos para modelos lineares generalizados são semelhantes às usadas para modelos lineares clássicos, com algumas adaptações (ATKINSON et al., 1989; MCCUllaGH \& NELDER, 1989 e Collet, 1991).

Supondo que um modelo linear generalizado é ajustado a $n$ observações $y_{i}{ }^{\prime} \mathrm{s}, i=1,2, \ldots, n$, e que $\hat{\mu}_{i}$ é o valor ajustado correspondente à observação $y_{i}$, então podem-se utilizar vários tipos de resíduos na verificação do ajuste desse modelo. Os mais comumente utilizados são descritos a seguir.

\section{- Resíduos de Pearson}

São dados por:

$$
X_{i}=\frac{\left(y_{i}-\hat{\mu}_{i}\right) \sqrt{w_{i}}}{\sqrt{V\left(\hat{\mu}_{i}\right)}}
$$

e são assim denominados porque, para as distribuições binomial e de Poisson, temse que seus quadrados constituem os componentes da estatística $X^{2}$ de Pearson, 
ou seja, $X^{2}=\sum_{i} X_{i}^{2}$, pois para essas distribuiçôes tem-se que $\hat{V}\left(Y_{i}\right)=V\left(\hat{\mu}_{i}\right) / w_{i}$ $(\phi=1)$. Portanto, para esses modelos este tipo de resíduo expressa a contribuição dada pela observação $y_{i}$ à estatística de Pearson, que é outra medida do ajuste de um modelo.

\section{- Resíduos de Pearson padronizados}

Os resíduos de Pearson, apesar de serem padronizados pela variância das observações, não têm variância constante, pois essa padronização não leva em conta a variância inerente aos valores ajustados $\hat{\mu}_{i}$. Uma maneira melhor de se padronizarem os resíduos ordinários é dividi-los pelas estimativas de seus erros padrões, ou seja, por $\sqrt{\hat{V}\left(Y_{i}-\hat{\mu}_{i}\right)}$. Isso é o mesmo que dividir os resíduos de Pearson por $\sqrt{\hat{\phi}\left(1-h_{i}\right)}$ (COLLET, 1991), onde $\hat{\phi}$ é a estimativa do parâmetro de escala, ou seu próprio valor, se conhecido; e $h_{i}$ é o i-ésimo elemento da diagonal da matriz $H$ de ordem $n$, tal que $H=\hat{W}^{1 / 2} X\left(X^{\prime} \hat{W} X\right)^{-1} X^{\prime} \hat{W}^{1 / 2}$, sendo $X$ a matriz $n \times p$ do modelo e $\hat{W}$ uma matriz $n \times n$ de pesos tal que

$$
\hat{W}=\operatorname{diag}\left\{\left.\frac{w_{i}}{V\left(\hat{\mu}_{i}\right)}\left(\frac{\mathrm{d} g^{-1}\left(\eta_{i}\right)}{\mathrm{d} \eta_{i}}\right)^{2}\right|_{\eta_{i}=\hat{\eta}_{i}}\right\} .
$$

Os resíduos de Pearson assim obtidos, aqui denotados por $r_{P_{i}}$ 's, são chamados de resíduos de Pearson padronizados e são dados por:

$$
r_{P_{i}}=\frac{y_{i}-\hat{\mu}_{i}}{\sqrt{\hat{V}\left(Y_{i}-\hat{\mu}_{i}\right)}}=\frac{\left(y_{i}-\hat{\mu}_{i}\right) \sqrt{w_{i}}}{\sqrt{\hat{\phi} V\left(\hat{\mu}_{i}\right)\left(1-h_{i}\right)}}=\frac{X_{i}}{\sqrt{\hat{\phi}\left(1-h_{i}\right)}} .
$$

\section{- Componentes de "deviance"}

Os componentes de "deviance", aqui denotados por $d_{i}$ 's, expressam a contribuição dada pelas observações $y_{i}$ 's à "deviance" residual do modelo e são dados por:

$$
d_{i}=\operatorname{sinal}\left(y_{i}-\hat{\mu}_{i}\right) \sqrt{2 w_{i}\left\{y_{i}\left[\tilde{\theta}_{i}-\hat{\theta}_{i}\right]-b\left(\tilde{\theta}_{i}\right)+b\left(\hat{\theta}_{i}\right)\right\}},
$$

onde sinal $\left(y_{i}-\hat{\mu}_{i}\right)$ é a função que faz $d_{i}$ positivo se $y_{i} \geq \hat{\mu}_{i}$ e negativo se $y_{i}<\hat{\mu}_{i}$, e $\tilde{\theta}_{i}$ e $\hat{\theta}_{i}$ são tais como na equação (25). 


\section{- Componentes de "deviance" padronizados}

Os componentes de "deviance" não têm variância unitária, uma vez que em sua obtenção não se considera a variação inerente a cada valor ajustado $\hat{\mu}_{i}$. No entanto, eles podem ser padronizados para que tenham variância aproximadamente igual a 1 , dando origem aos componentes de "deviance" padronizados ou componentes de "deviance" estudentizados internamente, aqui denotados por:

$$
r_{D_{i}}=\frac{\operatorname{sinal}\left(y_{i}-\hat{\mu}_{i}\right) \sqrt{d_{i}^{2}}}{\sqrt{\hat{\phi}\left(1-h_{i}\right)}}=\frac{d_{i}}{\sqrt{\hat{\phi}\left(1-h_{i}\right)}} .
$$

\section{- Componentes de "deviance" estudentizados externamente}

Outra maneira de se obter um resíduo é comparar as "deviances" obtidas pelo ajuste de um mesmo modelo ao conjunto de dados sob análise, com $n$ observações, e ao conjunto de dados com $(n-1)$ observações obtido ao se excluir a i-ésima observação do conjunto original de dados. Isto dá origem a uma quantia que mede a diferença na "deviance" quando cada observação é excluída do conjunto de dados sob análise. Valores exatos para essas estatísticas podem ser obtidos pelo ajuste do modelo aos $n$ dados e aos $n$ conjuntos de dados originados ao se omitir uma observação de cada vez. Esse procedimento, computacionalmente intensivo, pode ser evitado dado que, segundo CoLlet (1991), a mudança na "deviance" provocada pela omissão da i-ésima observação pode ser bem aproximada por:

$$
\left(1-h_{i}\right) r_{D_{i}}^{2}+h_{i} r_{P_{i}}^{2}
$$

cuja raiz quadrada, acompanhada do sinal da diferença $\left(y_{i}-\hat{\mu}_{i}\right)$, é o valor conhecido como componente de "deviance" estudentizado externamente, o qual, na literatura inglesa, recebe diferentes denominações, como "deletion residuals", "jacknifed residuals" e "likelihood residuals". Os componentes de "deviance" estudenizados externamente, aqui denotados por $r_{L_{i}}$ 's, podem, portanto, ser obtidos pelo cálculo de

$$
r_{L_{i}}=\operatorname{sinal}\left(y_{i}-\hat{\mu}_{i}\right) \sqrt{\left(1-h_{i}\right) r_{D_{i}}^{2}+h_{i} r_{P_{i}}^{2}}
$$


Vê-se que $r_{L_{i}}^{2}$ é uma combinação linear ponderada de $r_{D_{i}}^{2}$ e de $r_{P_{i}}^{2}$. Além disso, os valores de $r_{L}$ são próximos aos de $r_{D}$, pois, normalmente, os valores $\operatorname{dos} h_{i}$ 's são muito pequenos.

Verifica-se, facilmente, que para a distribuição normal os componentes de "deviance" padronizados, os resíduos de Pearson padronizados e os componentes de "deviance" estudentizados externamente são coincidentes. Já para as outras distribuições da família exponencial eles diferem entre si e não têm distribuição conhecida.

A análise de resíduos é em muito facilitada quando se conhece sua distribuição. Anscombe ${ }^{2}$, citado por Collet (1991), propôs um método para a construção de resíduos com distribuição aproximadamente normal padrão, os quais são conhecidos como resíduos de Anscombe.

\section{- Resíduos de Anscombe}

O método para obtenção de resíduos com distribuição aproximadamente normal padrão, proposto por Anscombe, consiste em encontrar uma função das observações, seja $A\left(y_{i}\right)$, que tenha distribuição aproximadamente normal e calcular

$$
r_{A_{i}}=\frac{A\left(y_{i}\right)-A\left(\hat{y}_{i}\right)}{\sqrt{\hat{V}\left[A\left(y_{i}\right)-A\left(\hat{y}_{i}\right)\right]}}
$$

de modo que $r_{A_{i}}$ assim obtido é chamado de resíduo de Anscombe.

Para a distribuição de Poisson, de acordo com McCullagh \& NELDER (1989), os resíduos de Anscombe são dados por:

$$
r_{A_{i}}=\frac{3 / 2\left(y_{i}^{2 / 3}-\hat{\mu}_{i}^{2 / 3}\right)}{\hat{\mu}_{i}^{1 / 6}} .
$$

\footnotetext{
${ }^{2}$ ANSCOMBE, F.J. Contribution to the discussion of a paper by H. Hotelling. Journal of the Royal Statistical Society B, 15: 229-30, 1953.
} 
Estudos numéricos realizados por Williams $^{3}$, citado por Collet (1991), e Pierce \& Shafer (1986) revelam que os componentes de "deviance" padronizados, os componentes de "deviance" estudentizados externamente e os residuos de Anscombe são muito similares entre si. Além disso, esses três tipos de resíduos são razoavelmente bem aproximados pela distribuição normal padrão. Por outro lado, segundo MCCullaGH \& Nelder (1989), os componentes de "deviance" são preferíveis aos resíduos de Pearson para os procedimentos de verificação do ajuste do modelo, pois suas propriedades são mais próximas daquelas dos resíduos usados em modelos de regressão linear.

Tendo em vista as considerações do parágrafo anterior, e dada a maior dificuldade prática em se trabalhar com os resíduos de Anscombe, neste trabalho são utilizados os componentes de "deviance" padronizados e os componentes de "deviance" estudentizados externamente.

Considerando-se as equações (6), (7) e (25), tem-se que os componentes de "deviance" para o modelo Poisson são dados por:

$$
d_{i}=\operatorname{sinal}\left(y_{i}-\hat{\lambda}_{i}\right) \sqrt{2 w_{i}\left[y_{i}\left(\ln y_{i}-\ln \hat{\lambda}_{i}\right)-y_{i}+\hat{\lambda}_{i}\right]}
$$

e, considerando-se também as equações (4) e (26), tem-se que os resíduos de Pearson padronizados, utilizados no cálculo dos componentes de "deviance" estudentizados externamente, para este modelo são dados por:

$$
r_{P_{i}}=\frac{\left(y_{i}-\hat{\lambda}_{i}\right) \sqrt{w_{i}}}{\sqrt{\hat{\lambda}_{i}\left(1-h_{i}\right)}}
$$

Para o modelo Poisson truncado, a partir das equações (1), (8) e (25), tem-se que os componentes de "deviance" são dados por:

$$
d_{i}=\operatorname{sinal}\left(y_{i}-\hat{\mu}_{i}\right) \sqrt{2 w_{i}\left[y_{i}\left(\ln \frac{\tilde{\lambda}}{\hat{\lambda}}\right)-\ln \frac{\left(e^{\tilde{\lambda}}-1\right)}{\left(e^{\hat{\lambda}}-1\right)},\right.},
$$

\footnotetext{
${ }^{3}$ WILLIAMS, D.A. Residuals in generalized linear models. In: INTERNACIONAL BIOMETRICS CONFERENCE, 12., Tokyo, 1984. Proceedings. Tokyo, 1984. p.59-68.
} 
onde $\tilde{\lambda}$ e $\hat{\lambda}$ são as estimativas de máxima verossimilhança para $\lambda$ sob os modelos saturado e corrente, respectivamente. Considerando-se também as equações (5), (10) e (26), tem-se que os resíduos de Pearson padronizados, utilizados no cálculo dos componentes de "deviance" estudentizados externamente, para o modelo Poisson truncado são dados por:

$$
r_{P_{i}}=\frac{\left(y_{i}-\hat{\mu}_{i}\right) \sqrt{w_{i}}}{\sqrt{\hat{\mu}_{i}\left(1+\hat{\lambda}_{i}-\hat{\mu}_{i}\right)\left(1-h_{i}\right)}} .
$$

Para o modelo binomial negativo, a partir das equações (21) e (25), tem-se que os componentes de "deviance" são dados por:

$$
d_{i}=\operatorname{sinal}\left(y_{i}-\hat{\mu}_{i}\right) \sqrt{2 w_{i}\left[y_{i} \ln \left(\frac{y_{i}}{\hat{\mu}_{i}}\right)-\left(y_{i}+k\right) \ln \left(\frac{y_{i}+k}{\hat{\mu}_{i}+k}\right)\right]},
$$

e, considerando-se também as equações (22) e (26), tem-se que os resíduos de Pearson padronizados, utilizados no cálculo dos componentes de "deviance" estudentizados externamente, para este modelo são dados por:

$$
r_{P_{i}}=\frac{\left(y_{i}-\hat{\mu}_{i}\right) \sqrt{k w_{i}}}{\sqrt{\hat{\mu}_{i}\left(k+\hat{\mu}_{i}\right)\left(1-h_{i}\right)}} .
$$

Embora as tabelas com os valores dos resíduos sejam informativas, a utilização de gráficos proporciona a visualização espacial dos resíduos, facilitando a verificação da adequabilidade do modelo. Existe uma variedade muito grande de gráficos comumente utilizados, tais como, resíduos versus índices, resíduos versus preditores lineares, gráficos normais de probabilidade para os resíduos ("normal plots"), gráficos normais de probabilidade para os valores absolutos dos resíduos ("half normal plots"), etc.

\section{- Gráfico de resıduos versus índices}

Consiste no gráfico dos resíduos contra os números das respectivas observações, ou índices, sendo conhecido como gráfico de índice. Seu padrão ideal é dado por uma "nuvem" de pontos espalhados entre as ordenadas -2 e +2 . É adequado para detectar "outliers", aquelas observações cujos pontos no gráfico aparecem 
deslocados dos demais abaixo e acima, respectivamente, das retas de ordenadas iguais a -2 e +2 .

- Gráfico de residuos versus tratamentos ordenados pelas médias

É adequado para verificar se existe homogeneidade de variância entre os resíduos, ou seja, se o modelo está explicando toda a variação contida nos dados. Seu padrão ideal é dado por pontos distribuídos entre as ordenadas -2 e +2 em todas as abscissas, de modo que esses pontos formem uma faixa ou banda horizontal, isto é, para todos os tratamentos os resíduos devem ter a mesma amplitude de variação.

- Gráfico normal de probabilidade para os residuos ("normal plot") e gráfico normal de probabilidade para os valores absolutos dos residuos ("half normal plot")

Os gráficos normais de probabilidade podem ser utilizados na verificação do ajuste de um modelo linear generalizado com base no resultado, já mencionado, que garante que a distribuição dos componentes de "deviance" padronizados pode ser aproximada pela distribuição normal padrão quando o modelo é correto. A idéia que dá origem a esses gráficos é que, colocando-se os resíduos em ordem crescente e plotando-os contra uma aproximação de seus valores esperados, calculada com base na pressuposição de que eles seguem a distribuição normal, então se tal pressuposição está correta o gráfico obtido deve ser, aproximadamente, uma linha reta. Os valores esperados dos residuos podem ser aproximados pelo probito de $\Phi^{-1}\left\{\left(i-\frac{3}{8}\right) /\left(n+\frac{1}{4}\right)\right\}\left(\right.$ Blom $^{4}$, citado por Collet, 1991), onde $i$ é o índice do i-ésimo resíduo após efetuada a ordenação, ou seja, denotando-se os valores ordenados dos $n$ resíduos por $r_{(i)}$ 's, tem-se que $r_{(i)}<r_{\left(i^{\prime}\right)} \Leftrightarrow i<i^{\prime}$, para qualquer par $i, i^{\prime}$.

Este tipo de gráfico pode revelar a falta de ajuste do modelo e/ou a presença de "outliers". No entanto, os padrões que revelam essas características do ajuste ficam mais evidenciados num gráfico normal de probabilidade para os valores

${ }^{4}$ BLOM, G. Statistical estimates and transformed beta-variables. New York, Wiley, 1958. 
absolutos dos resíduos, gráfico em que estes valores absolutos são arranjados em ordem crescente e plotados contra os valores dados por: $\Phi^{-1}\left\{\left(i+n-\frac{1}{8}\right) /\left(2 n+\frac{1}{2}\right)\right\}$, onde $i$ é o índice do i-ésimo resíduo após a ordenação. Neste caso, os "outliers" apareceriam na parte superior direita do gráfico como pontos distanciados da suposta reta, e a falta de ajuste do modelo se revelaria quando o gráfico obtido não se aproximasse de uma reta. Entretanto, como os resíduos usados na construção do gráfico são correlacionados, pode ocorrer que ele resulte numa linha que não se aproxima de uma reta mesmo quando o modelo é correto. Por isso, a interpretação dos gráficos normais de probabilidade fica muito facilitada pela construção de um envelope simulado conforme foi proposto por ATKINSON (1981). Este envelope é tal que, se o modelo estiver correto, os pontos plotados ficam todos dentro de seus limites.

Para o gráfico normal de probabilidade para os valores absolutos dos componentes de "deviance" padronizados do modelo Poisson, por exemplo, o envelope simulado é construf́do da seguinte maneira.

(a) Para cada observação $y_{i}, i=1,2, \ldots, n$, simulam-se 19 observações de uma distribuição de Poisson com parâmetro $\hat{\lambda}_{i}$, onde $\hat{\lambda}_{i}$ é a estimativa do parâmetro $\lambda$ obtida para a observação $y_{i}$;

(b) ajusta-se, a cada um dos 19 conjuntos de dados simulados, o modelo ajustado aos dados originais;

(c) de cada um desses 19 ajustes, obtêm-se os valores absolutos dos componentes de "deviance" padronizados, ou seja, os $\left|r_{D_{i}}\right|$ 's, e arranja-se-os em ordem crescente de forma que $\left|r_{D}\right|_{(1)}<\left|r_{D}\right|_{(2)}<\cdots<\left|r_{D}\right|_{(n)}$;

(d) para cada $\left|r_{D}\right|_{(i)}$ calculam-se a média, o mínimo, e o máximo dos 19 valores estimados para essa estatística nos 19 ajustes efetuados, um para cada conjunto de dados simulado;

(e) essas estatísticas, ou seja, a média, o mínimo, e o máximo calculados para cada $i$, são, então, plotadas num gráfico normal de probabilidade junto 
aos valores absolutos dos componentes de "deviance" padronizados resultantes do ajuste do modelo aos dados originais, gerando, respectivamente, a linha média e os limites inferior e superior do envelope.

Com o uso de 19 simulações tem-se que a chance de que o valor de cada $\left|r_{D}\right|_{(i)}$ do conjunto de dados original fique fora do envelope simulado quando o modelo ajustado está correto é de 1 em 20 , ou de $5 \%$. Este resultado pode ser usado para assegurar se a observação que produz o maior resíduo pode ser considerada uma observação discrepante. Entretanto, a maior vantagem do envelope simulado é que o gráfico normal de probabilidade pode ser interpretado sem se ter que fazer alguma suposição a respeito da distribuição dos resíduos. Em particular, pontos que se distanciam da linha média ou que ficam próximos ou fora dos limites do envelope, indicam que o modelo ajustado não é apropriado.

CollET (1991) apresenta um programa em GLIM para a construção do gráfico normal de probabilidade para os valores absolutos dos componentes de "deviance" padronizados com envelope simulado para o modelo binomial. No Apêndice 11 apresenta-se uma adaptação deste programa, feita para o modelo Poisson. O método aqui utilizado para a simulação de dados com distribuição de Poisson (Morgan, 1986) é descrito a seguir.

(a) Supõe-se $\left\{E_{h}, h \geq 1\right\}$ uma seqüência de variáveis explanatórias independentes e identicamente distribuídas, todas com distribuição exponencial com parâmetro $\lambda$, cuja função densidade de probabilidade é dada por:

$$
f_{E_{h}}(x ; \lambda)=\lambda e^{-\lambda x} I_{[0, \infty)}, \quad \lambda>0,
$$

onde $x$ é um valor assumido por $E_{h}$;

(b) definem-se $S_{0}=0$ e $S_{n}=\sum_{h=1}^{n} E_{h}$, para $n \geq 1$, de forma que a variável aleatória $K$ definida implicitamente pelas desigualdades $S_{K} \leq 1<S_{K+1}$ tem distribuição de Poisson com parâmetro $\lambda$; 
(c) toma-se $S_{1}=E_{1}$. Se $S_{1}>1$, então $S_{0}=0 \leq 1<S_{1}$ e toma-se $K=0$. Se, ao contrário, $S_{1} \leq 1$, então toma-se $S_{2}=E_{1}+E_{2}$ e se, por sua vez, $S_{2}>1$, tem-se que $S_{1} \leq 1<S_{2}$ e toma-se $K=1$. Se, por outro lado, $S_{2} \leq 1$, então toma-se $S_{3}=E_{1}+E_{2}+E_{3}$, e assim por diante até que $n$ seja tal que $S_{n} \leq 1<S_{n+1}$, e então toma-se $K=n$. A variável aleatória $K$ assim definida tem distribuição de Poisson com parâmetro $\lambda$.

De fato, se $E_{h}$, para qualquer que seja $h, h \geq 1$, é uma variável aleatória com distribuição exponencial com parâmetro $\lambda$, ou seja, $E_{h} \sim \exp (\lambda)$, e se $S_{n}=\sum_{h=1}^{n} E_{h}$, então tem-se que

$$
\begin{gathered}
E\left(E_{h}\right)=\frac{1}{\lambda} \\
S_{n} \sim \Gamma(n, \lambda) \\
E\left(S_{n}\right)=\frac{n}{\lambda},
\end{gathered}
$$

e é de se esperar que sejam necessários $(K+1) E_{h}$ 's, onde $K$ é o número natural dado pela parte inteira de $\lambda$, para se ter $S_{K} \leq 1<S_{K+1}$, pois

$$
\begin{gathered}
E\left(S_{n}=S_{K}\right)=\frac{K}{\lambda} \leq 1, \text { e } \\
E\left(S_{n}=S_{K+1}\right)=\frac{K+1}{\lambda}>1 .
\end{gathered}
$$

Portanto, a variável aleatória $K$ definida de acordo com o método exposto tem distribuição de Poisson com parâmetro $\lambda$.

Este método consiste, então, em se gerar uma variável aleatória com distribuição de Poisson com parâmetro $\lambda$ a partir de variáveis aleatórias com distribuição exponencial com parâmetro $\lambda$. Estas, por sua vez, são geradas a partir de variáveis aleatórias contínuas, com distribuição uniforme com parâmetros 0 e 1 , pois, dada uma variável aleatória $U$ assim definida, ou seja, se $U \sim \mathrm{U}(0,1)$, então sabe-se que a variável aleatória $E$ dada por:

$$
E=-\frac{1}{\lambda} \log U
$$

tem distribuição exponencial com parâmetro $\lambda$. 


\section{RESULTADOS E DISCUSSÃO}

A seguir, apresentam-se os resultados obtidos para cada conjunto de dados e sua respectiva discussão.

\section{Conjunto A}

O resultado do teste do cociente variância-média, apresentado na Tabela 1, indica que, com exceção dos tratamentos $T_{32}$ e $T_{33}$, que tiveram todas as observaçõs iguais a zero, e dos tratamentos $T_{22}$ e $T_{23}$, para os quais o teste foi significativo ao nível de $5 \%$ de probabilidade, para todos os demais tratamentos aceita-se a hipótese de que as observações seguem a distribuição de Poisson.

Tabela 1: Teste do cociente variância-média aplicado aos tratamentos do Conjunto A de dados.

\begin{tabular}{ccrcc}
\hline Tratamento & $n$ & \multicolumn{1}{c}{$\bar{y}$} & $s^{2}$ & $I(n-1)$ \\
\hline 11 & 14 & 10,07 & 4,99 & $6,45^{n s}$ \\
12 & 14 & 0,86 & 1,05 & $16,00^{n s}$ \\
13 & 14 & 0,14 & 0,13 & $12,00^{n s}$ \\
21 & 15 & 9,00 & 5,00 & $7,78^{n s}$ \\
22 & 14 & 0,36 & 0,86 & $31,40^{*}$ \\
23 & 14 & 0,14 & 0,28 & $26,00^{*}$ \\
31 & 14 & 0,28 & 0,37 & $17,00^{n s}$ \\
32 & 14 & 0,00 & 0,00 & - \\
33 & 14 & 0,00 & 0,00 & - \\
\hline
\end{tabular}

$$
\chi_{(0,975 ; 13)}^{2}=5,01 \quad \chi_{(0,025 ; 13)}^{2}=24,75 \quad \chi_{(0,975 ; 14)}^{2}=5,63 \quad \chi_{(0,025 ; 14)}^{2}=26,14
$$


Em conformidade com este resultado, o teste para detectar superdispersão não foi significativo ao nível de $5 \%$ de probabilidade, o que indica a não ocorrência de superdispersão no modelo Poisson. O valor calculado para a estatística $T_{a}$ do teste e o valor crítico da distribuição normal padrão para $\alpha=0,05$ são:

$$
T_{a}=-1,51^{n s} \quad Z_{0,05}=1,64 .
$$

Ajustando-se o modelo Poisson, descrito no item 3.2, a este conjunto de dados, os resultados obtidos na análise de "deviance" apresentam-se na Tabela 2. De acordo com esses resultados, o teste de $\chi^{2}$ para a "deviance" residual do modelo, associada a 118 graus de liberdade, não foi significativo ao nível de $5 \%$ de probabilidade, ou seja, não rejeitou o modelo. Este resultado é coerente com aqueles dos testes para a distribuição dos dados e para detectar superdispersão.

Tabela 2: Resultados da análise de "deviance" para a variável número de calos produzidos por explante, do Conjunto A de dados, usando o modelo Poisson.

\begin{tabular}{|c|c|c|c|c|}
\hline Fontes de Variação & GL & "Deviance" & "Deviance" média & Razão \\
\hline Radiação (R) & 2 & 205,77 & 102,88 & $144,90^{* *}$ \\
\hline ANA $(\mathrm{A})$ & 2 & 477,00 & 238,50 & $335,91^{* *}$ \\
\hline $\mathrm{R} \times \mathrm{A}$ & 4 & 2,73 & 0,68 & $0,96^{n s}$ \\
\hline Resíduo & 118 & $83,95^{n s}$ & 0,71 & \\
\hline Total & 126 & 769,45 & & \\
\hline \multicolumn{5}{|c|}{$\chi_{(0,05 ; 118)}^{2}=144,60$} \\
\hline \multicolumn{3}{|c|}{$F_{(0,05 ; 2,118)}=3,07$} & $F_{(0,05 ; 4,118)}=2,45$ & \\
\hline \multicolumn{3}{|c|}{$F_{(0,01 ; 2,118)}=4,79$} & $F_{(0,01 ; 4,118)}=3,48$ & \\
\hline
\end{tabular}

Examinando-se o gráfico dos componentes de "deviance" padronizados versus índices resultante deste ajuste, apresentado na Figura 1, verifica-se que o 
mesmo está relativamente próximo de seu padrão ideal caracterizado por uma "nuvem" de pontos espalhados entre as ordenadas -2 e +2 . Os pontos situam-se entre as ordenadas $-1,44$ e $+2,84$, sendo que somente três deles aparecem acima da reta de ordenada igual a +2 , deslocados dos demais. Tais pontos correspondem às observações de índices 60,72 , e 94 , e referem-se a contagens de 2 ou 3 calos por explante observadas em tratamentos que tiveram no mínimo 11 observações iguais a zero (os tratamentos $T_{22}, T_{23}$, e $T_{31}$, respectivamente). As mesmas considerações podem ser feitas para o gráfico dos componentes de "deviance" estudentizados externamente versus índices, apresentado na Figura 2.

O gráfico dos componentes de "deviance" padronizados versus tratamentos ordenados pelas médias, apresentado na Figura 3, não satisfaz seu padrão ideal, pois, a não ser pelos pontos correspondentes aos tratamentos que tiveram as menores médias, $T_{32}$ e $T_{33}$, este gráfico apresenta uma tendência linear, ou seja, à medida que a média dos tratamentos aumenta, o intervalo dos valores assumidos pelos residuos desloca-se para o sentido negativo do eixo das ordenadas. Isto indica existência de correlação entre os componentes de "deviance" padronizados resultantes deste ajuste. No entanto este gráfico isoladamente não é conclusivo, pois não resultou num padrão bem definido, dada a coincidência dos pontos correspondentes aos tratamentos $T_{32}$ e $T_{33}$. Estes tratamentos tiveram todas as observações iguais a zero, daf́ a coincidência de seus pontos no gráfico. Na Tabela 19, que consta do Apêndice 12, apresentam-se os tratamentos ordenados pelas médias.

O gráfico normal de probabilidade para os valores absolutos dos componentes de "deviance" padronizados com envelope simulado, apresentado na Figura 4 , resultou próximo de seu padrão ideal, mas apresenta dois pontos distanciados da linha média na parte superior direita do gráfico, o que os indica como "outliers". Esses pontos correspondem às observações de índices 60 e 72, que também aparecem como "outliers" nos gráficos de índices.

Considerando-se, alternativamente, o modelo Poisson truncado para este conjunto de dados, o resultado obtido na análise de "deviance", apresentado na 


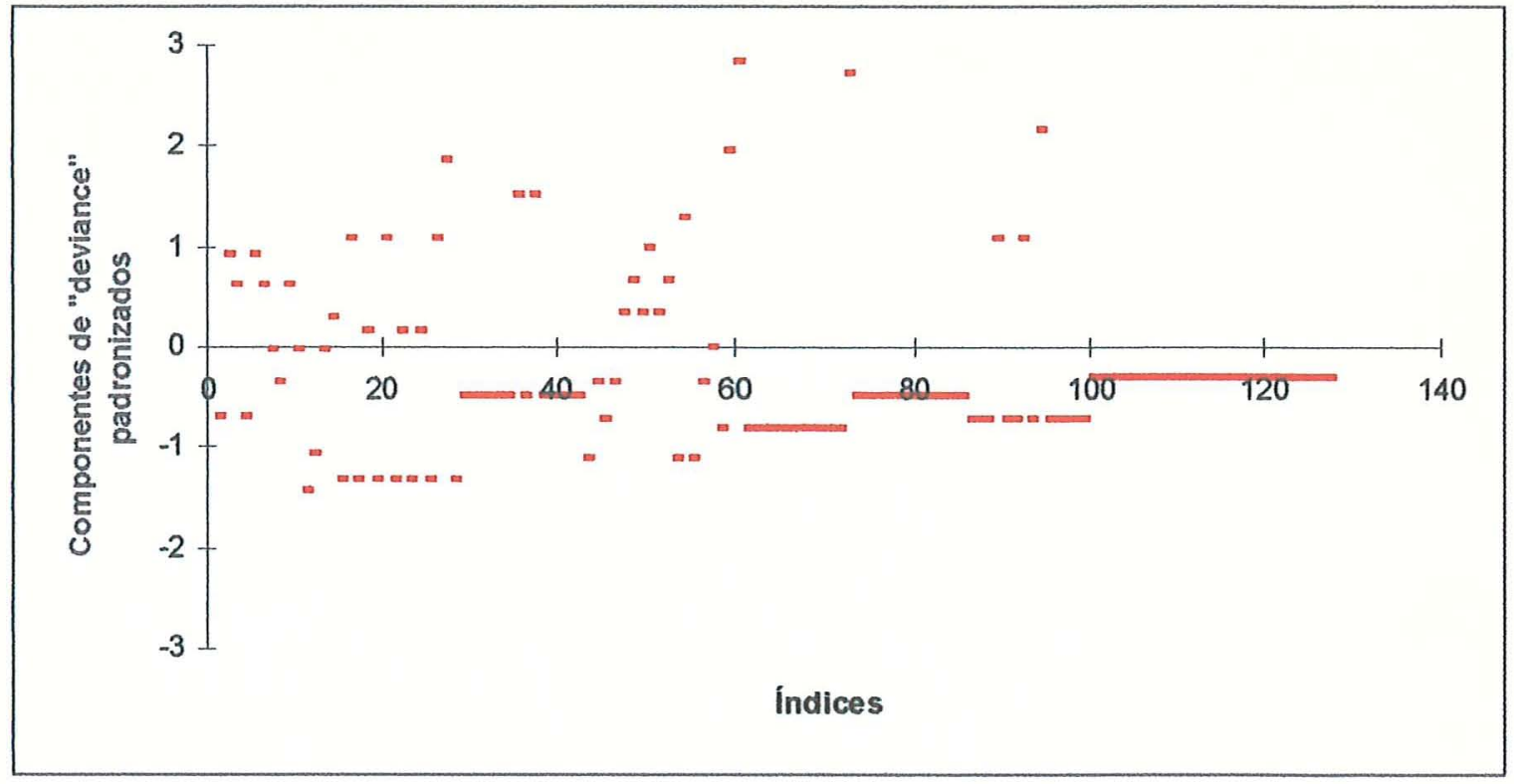

Figura 1: Gráfico dos componentes de "deviance" padronizados versus índices, resultante do ajuste do modelo Poisson ao Conjunto $\mathrm{A}$ de dados.

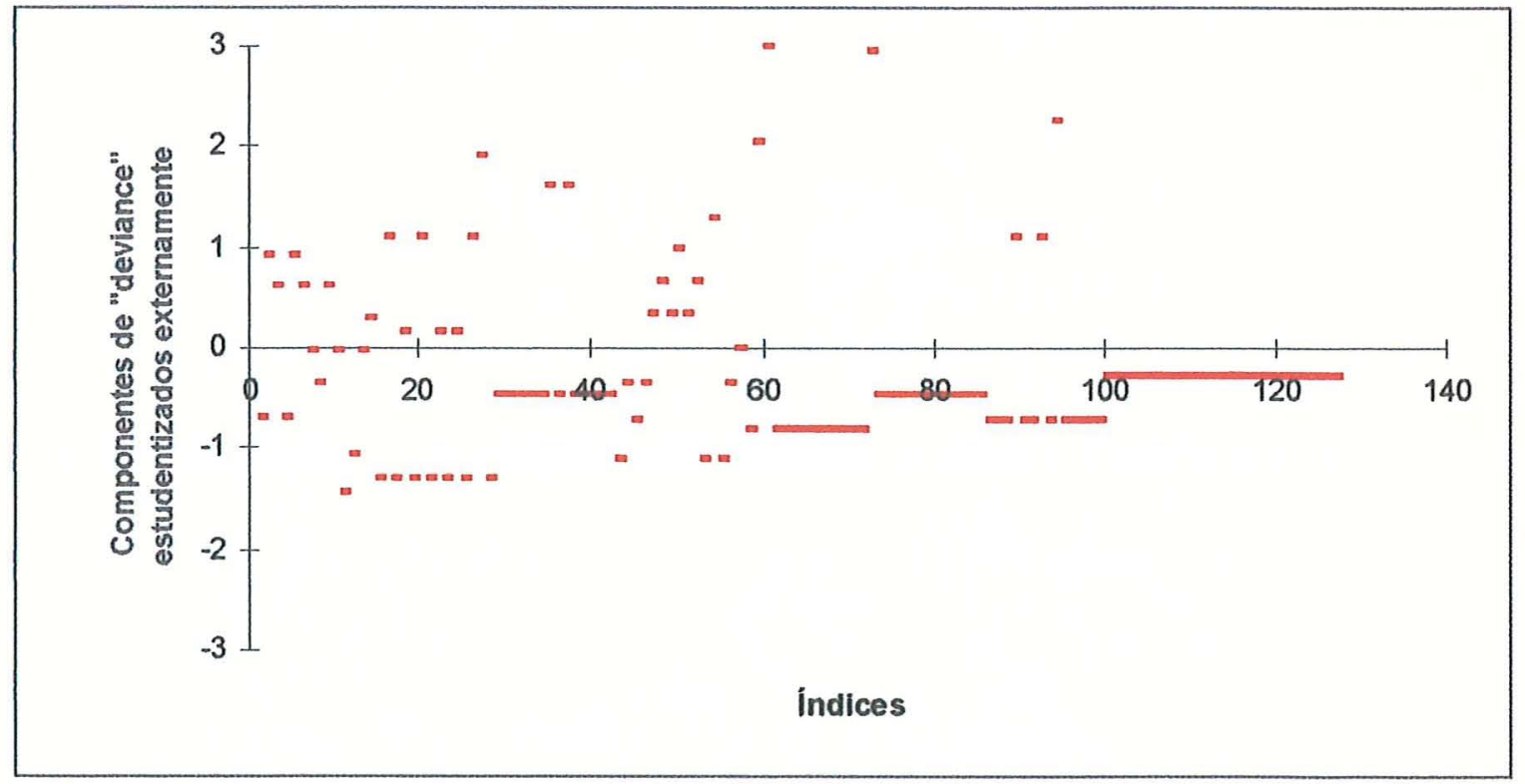

Figura 2: Gráfico dos componentes de "deviance" estudentizados externamente versus índices, resultante do ajuste do modelo Poisson ao Conjunto A de dados. 


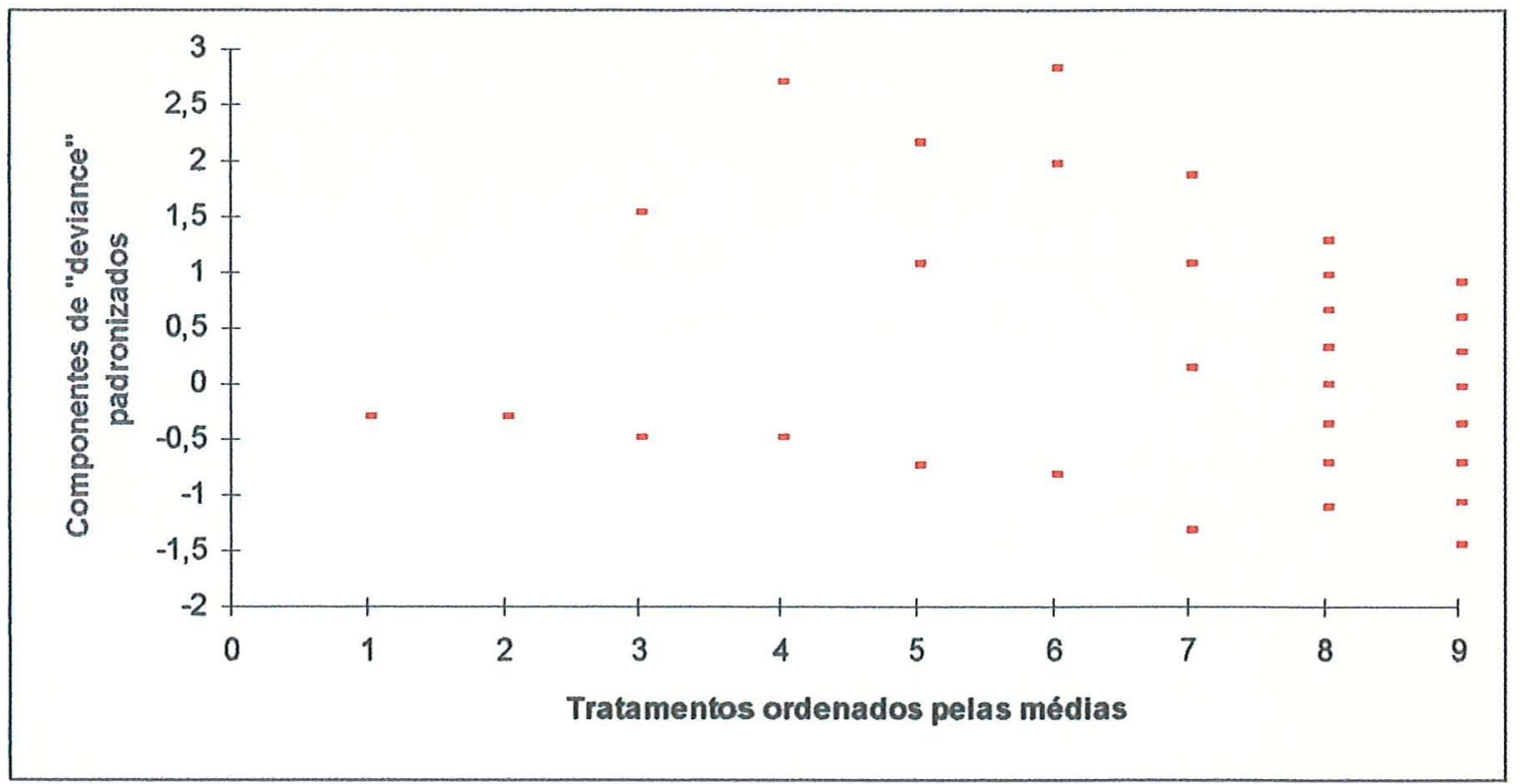

Figura 3: Gráfico dos componentes de "deviance" padronizados versus tratamentos ordenados pelas médias, resultante do ajuste do modelo Poisson ao Conjunto A de dados.

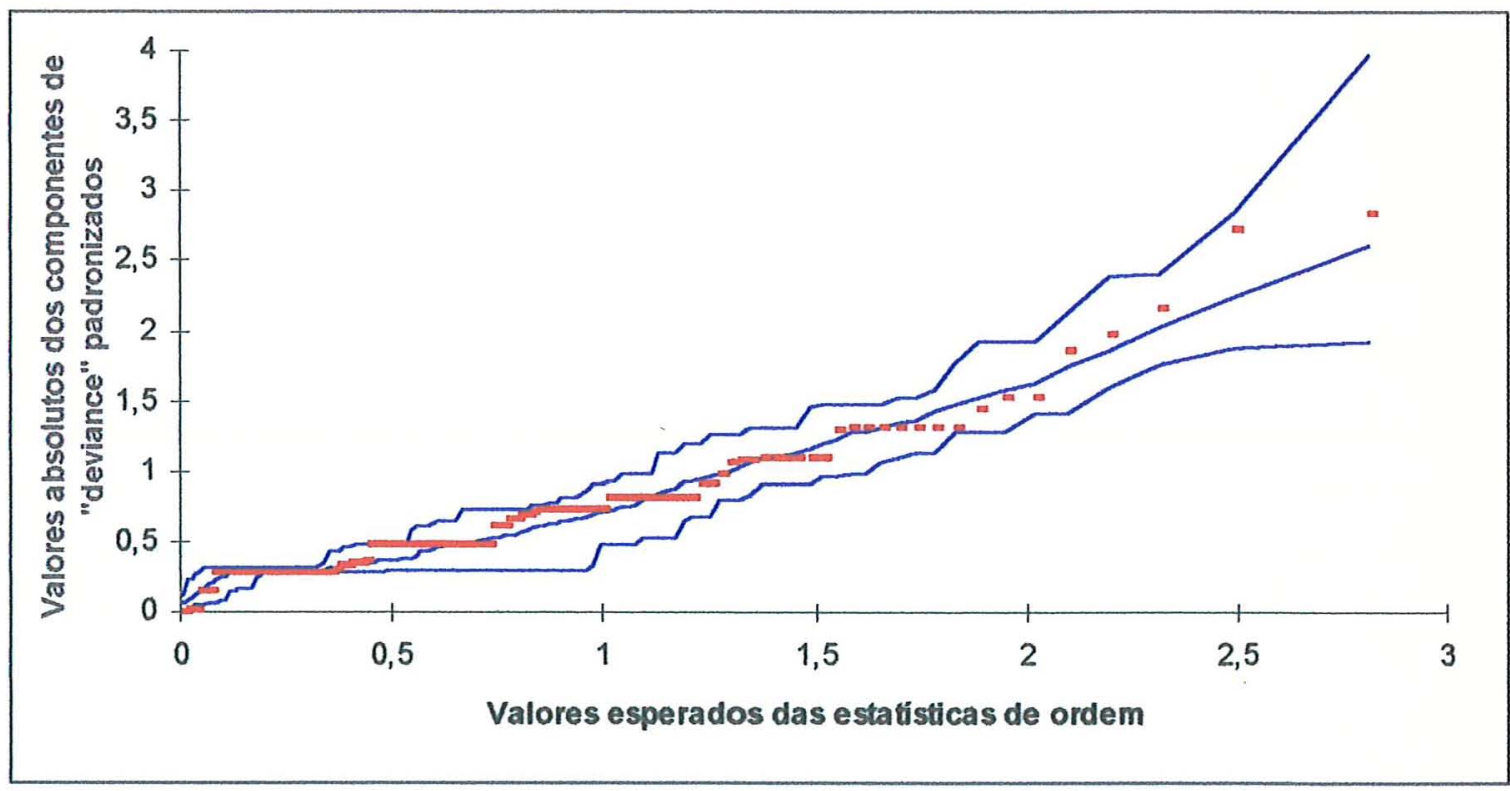

Figura 4: Gráfico normal de probabilidade para os valores absolutos dos componentes de "deviance" padronizados, resultante do ajuste do modelo Poisson ao Conjunto A de dados. 
Tabela 3, mostra que o teste de $\chi^{2}$ para a "deviance" residual do modelo, associada a 37 graus de liberdade, não foi significativo ao nível de $5 \%$ de probabilidade, indicando que o ajuste foi bom.

Tabela 3: Resultados da análise de "deviance" para a variável número de calos produzidos por explante, do Conjunto A de dados, usando o modelo Poisson truncado.

\begin{tabular}{lrccc}
\hline Fontes de Variação & GL & "Deviance" & "Deviance" média & Razão \\
\hline Radiação (R) & 2 & 25,50 & 12,75 & $21,25^{* *}$ \\
ANA (A) & 2 & 96,51 & 48,25 & $80,42^{* *}$ \\
R x A & 2 & 3,19 & 1,59 & $2,65^{n s}$ \\
Resíduo & 37 & $22,33^{n s}$ & 0,60 & \\
\hline Total & 43 & 147,53 & & \\
\hline
\end{tabular}

$$
\begin{gathered}
\chi_{(0,05 ; 37)}^{2}=52,28 \\
F_{(0,05 ; 2,37)}=3,25 \quad F_{(0,01 ; 2,37)}=5,23
\end{gathered}
$$

Os gráficos dos componentes de "deviance" padronizados versus índices e dos componentes de "deviance" estudentizados externamente versus índices resultantes deste ajuste, apresentados nas Figuras 5 e 6, respectivamente, apresentam os pontos bem espalhados entre as ordenadas $-1,5$ e $+1,5$; satisfazendo o padrão ideal deste tipo de gráfico.

O gráfico dos componentes de "deviance" padronizados versus tratamentos ordenados pelas médias, apresentado na Figura 7, salvo pelos pontos correspondentes aos tratamentos que tiveram a menor e a quarta menor médias, $T_{13} \mathrm{e}$ $T_{23}$, respectivamente, mostra-se relativamente próximo de seu padrão ideal. Na Tabela 20, que consta do Apêndice 13, apresentam-se os tratamentos ordenados pelas médias. O tratamento $T_{13}$ teve duas observações iguais a um, daí a coincidência de seus pontos no gráfico, e o tratamento $T_{23}$ teve uma única observação, apresentando 


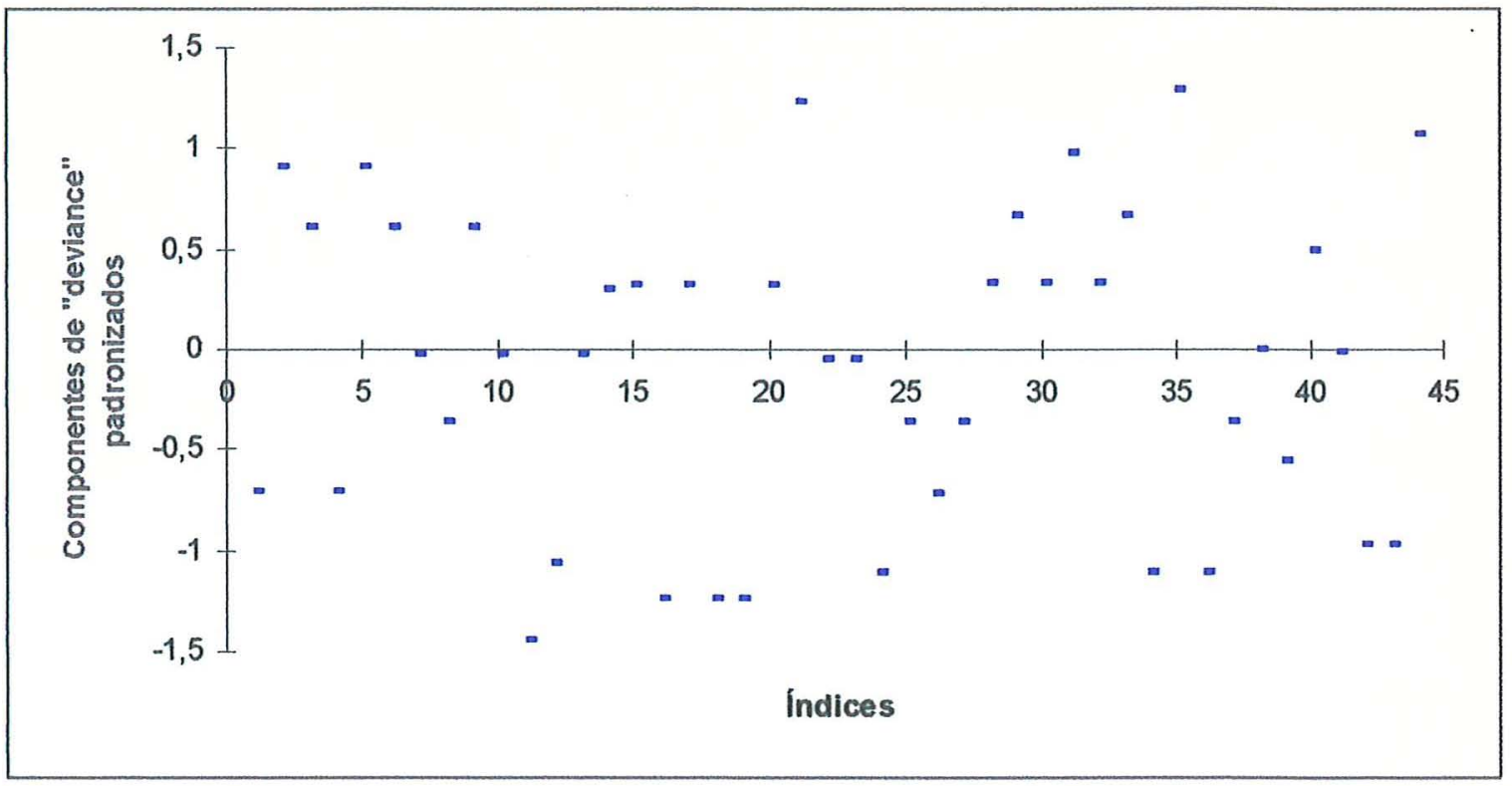

Figura 5: Gráfico dos componentes de "deviance" padronizados versus índices, resultante do ajuste do modelo Poisson truncado ao Conjunto A de dados.

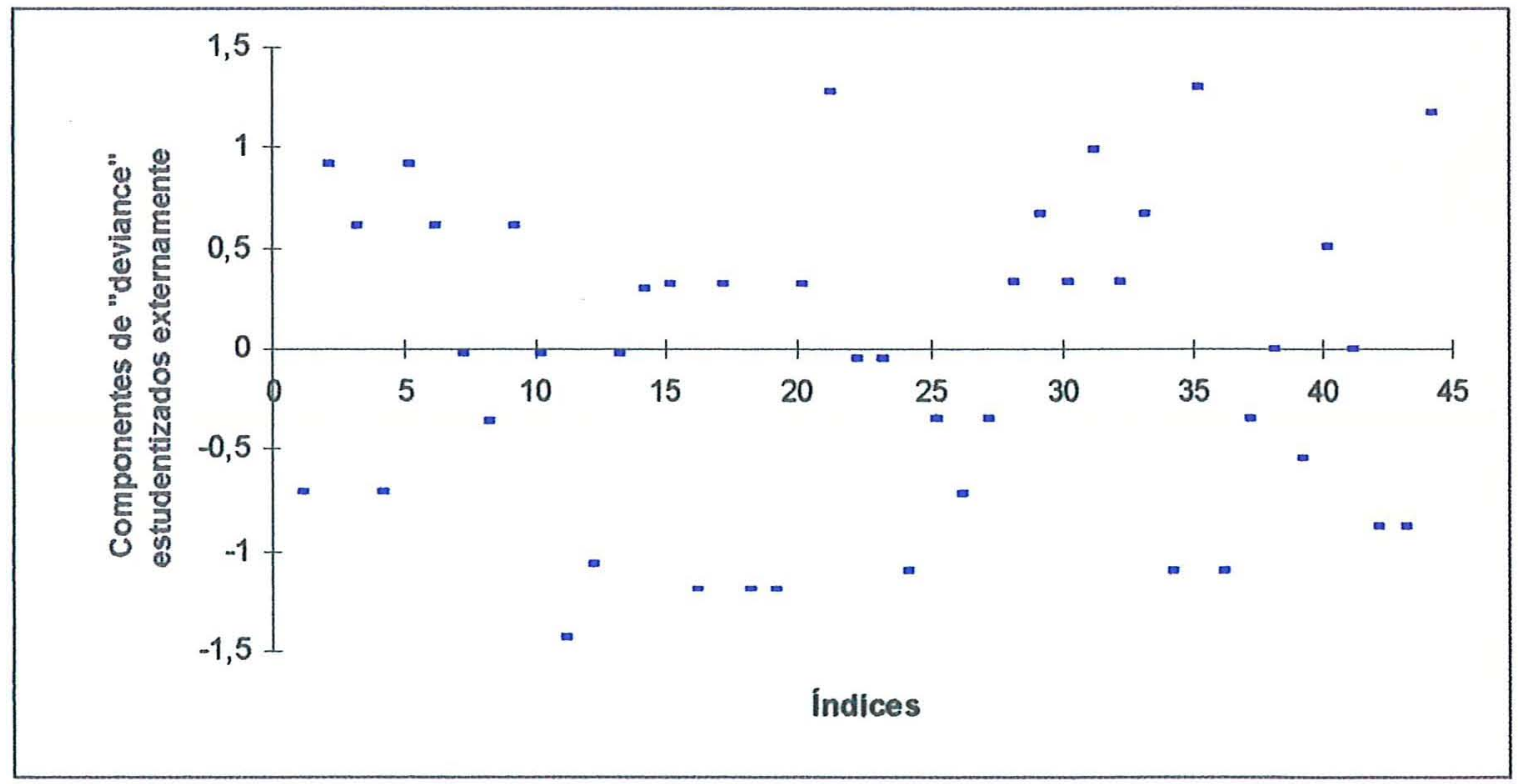

Figura 6: Gráfico dos componentes de "deviance" estudentizados externamente versus índices, resultante do ajuste do modelo Poisson truncado ao Conjunto $\mathrm{A}$ de dados. 


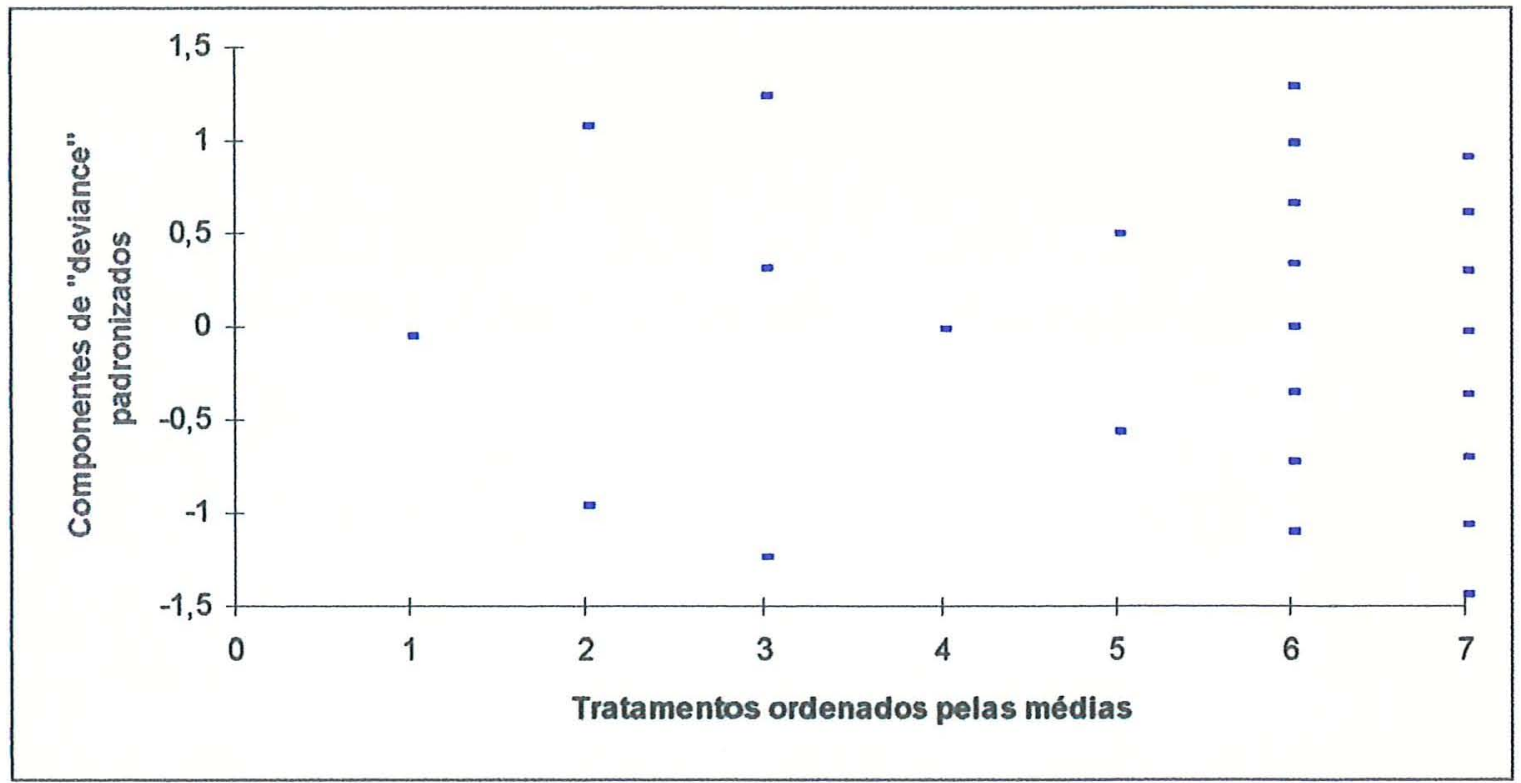

Figura 7: Gráfico dos componentes de "deviance" padronizados versus tratamentos ordenados pelas médias, resultante do ajuste do modelo Poisson truncado ao Conjunto A de dados. 
só um ponto no gráfico. Portanto, assim como para o modelo Poisson, este gráfico não resultou num padrão bem definido, e, isoladamente, não é conclusivo.

Tendo em vista os resultados do teste do cociente variância-média e do teste para superdispersão, o modelo binomial negativo não foi ajustado a este conjunto de dados por tratar-se de uma alternativa para o modelo Poisson com superdispersão.

Dentre os dois modelos ajustados, embora nenhum deles tenha sido rejeitado pelo teste de $\chi^{2}$ para a "deviance" residual do modelo, considerando-se a análise gráfica, aquele que melhor se ajustou aos dados foi o modelo Poisson truncado.

Conforme o exposto nos ítens 2.2 e 2.3, o modelo Poisson oferece conclusões a respeito do número médio de calos produzidos por explante, ao passo que, sob o modelo Poisson truncado, as conclusões obtidas referem-se ao número médio de calos produzidos por explante, dado que o explante regenera.

As médias dos tratamentos para o número de calos produzidos por explante consideradas sob os modelos Poisson e Poisson truncado, ou seja, o número médio de calos produzidos por explante e o número médio de calos produzidos por explante, dado que o explante regenerou, apresentam-se no Quadro 4, acompanhadas dos números de repetições dos tratamentos sob esses dois modelos.

Considerando-se para este conjunto de dados o modelo que teve o melhor ajuste, ou seja, o modelo Poisson truncado, tem-se que os resultados do teste $F$ aproximado para as fontes de variação do modelo apresentam-se na Tabela 3. Uma vez que o efeito de interação entre os fatores estudados não foi significativo ao nível de $5 \%$ de probabilidade, considerou-se o resultado do teste $F$ aproximado para o efeito principal desses fatores.

Conforme se verifica na Tabela 3 , este teste foi significativo ao nível de $1 \%$ de probabilidade tanto para o efeito do fator tipo de radiação luminosa como para o efeito do fator ANA. A partir deste resultado, o interesse é verificar como os níveis de cada fator diferem entre si. 
Quadro 4: Médias dos tratamentos do Conjunto A de dados para a variável número de calos produzidos por explante considerada sob os modelos Poisson e Poisson truncado, acompanhadas dos números de repetições dos tratamentos $(n)$.

\begin{tabular}{|c||c|c|c|c|}
\hline \multirow{2}{*}{\multicolumn{1}{|c||}{ Tratamento }} & \multicolumn{4}{c|}{ Modelo } \\
\cline { 2 - 5 } \multicolumn{1}{||}{} & \multicolumn{2}{|c|}{ Poisson } & \multicolumn{2}{c|}{ Poisson truncado } \\
\cline { 2 - 5 } & Média & $n$ & Média & $n$ \\
\hline \hline \multirow{2}{*}{11} & 10,07 & 14 & 10,07 & 14 \\
12 & 0,85 & 14 & 1,71 & 7 \\
13 & 0,14 & 14 & 1,00 & 2 \\
21 & 9,00 & 15 & 9,00 & 15 \\
22 & 0,35 & 14 & 2,50 & 2 \\
23 & 0,14 & 14 & 2,00 & 1 \\
31 & 0,28 & 14 & 1,33 & 3 \\
32 & 0,00 & 14 & - & - \\
33 & 0,00 & 14 & - & - \\
\hline
\end{tabular}

Para o fator tipo de radiação luminosa, os níveis foram comparados desdobrando-se os dois graus de liberdade correspondentes a este fator em dois contrastes ortogonais de interesse, a saber:

1. lâmpada branca fria e lâmpada de amplo espectro comparadas com escuro, ou seja, níveis 1 e 2 versus nível 3 ;

2. lâmpada branca fria comparada com lâmpada de amplo espectro, ou seja, nível 1 versus nível 2.

Para o fator ANA foi realizado um estudo de regressão, desdobrandose seu efeito em efeitos linear e quadrático. 
Os resultados obtidos na análise de "deviance" correspondente a esses desdobramentos apresentam-se na Tabela 4, e o programa em GLIM utilizado para efetuar esta análise apresenta-se no Apêndice 5.

Tabela 4: Resultados da análise de "deviance" para a variável número de calos produzidos por explante, do Conjunto A de dados, para estudo dos efeitos principais dos fatores, usando o modelo Poisson truncado.

\begin{tabular}{lcccc}
\hline Fontes de Variação & GL & "Deviance" & "Deviance" média & Razão \\
\hline (R1 + R2) vs R3 & 1 & 23,66 & 23,66 & $39,43^{* *}$ \\
R1 vs R2 & 1 & 1,84 & 1,84 & $3,07^{\text {ns }}$ \\
(Radiação) & $(2)$ & $(25,50)$ & & \\
Linear & 1 & 95,63 & 95,63 & $159,38^{* *}$ \\
Quadrático & 1 & 0,88 & 0,98 & $1,47^{n s}$ \\
(ANA) & $(2)$ & $(96,51)$ & & \\
Resíduo & 37 & 22,33 & 0,60 & \\
\hline Total & 43 & 147,53 & & \\
\hline \multicolumn{5}{c}{$F_{(0,05 ; 1,37)}$} \\
\end{tabular}

Para o fator tipo de radiação luminosa tem-se que as lâmpadas branca fria e de amplo espectro não diferem entre si ao nível de $5 \%$ de probabilidade, e, conjuntamente, diferem do nível escuro ao nível de $1 \%$ de probabilidade. Conforme se verifica no Quadro 4, que apresenta as médias dos tratamentos para a variável estudada, o número médio de calos produzidos por explante de Stylosanthes scabra, dado que o explante regenerou, é maior na presença de luz do que no escuro. Para o fator ANA, o efeito quadrático não foi significativo, ao nível de $5 \%$ de probabilidade, ao passo que o efeito linear foi significativo ao nível de $1 \%$ de probabilidade.

Com base nos resultados discutidos no parágrafo anterior, as lâmpadas branca fria e de amplo espectro foram consideradas como um único tratamento, 
para o qual foi feita uma regressão linear em função da dose de ANA. O procedimento utilizado para efetuar esta regressão faz parte do programa apresentado no Apêndice 5.

A equação de regressão estimada, ou seja, o preditor linear estimado, foi:

$$
\hat{\eta}_{1 j}=\hat{\beta}_{0}+\hat{\beta}_{1} x_{j}=2,14-4,19 x_{j}, \quad j=1,2,3
$$

onde $\beta_{0}$ e $\beta_{1}$ são os coeficientes linear e angular, respectivamente, da equação de regressão linear e $x_{j}$ representa a dose de ANA no meio de cultura, em mg/l, ou seja, $x_{1}=0,0 ; x_{2}=0,5$ e $x_{3}=1,0$.

A estimativa obtida para a matriz das variâncias e covariâncias de $\hat{\beta}=\left(\hat{\beta}_{0}, \hat{\beta}_{1}\right)^{\prime}$, associadas a 39 graus de liberdade, foi a seguinte:

$$
\hat{V}(\hat{\beta})=\left[\begin{array}{rr}
0,004528 & -0,008327 \\
-0,008327 & 0,004528
\end{array}\right]
$$

$\mathrm{O}$ valor estimado, $\hat{\mu}_{1 j}$, para o número médio de calos produzidos por explante de Stylosanthes scabra, dado que o explante regenerou, na presença de lâmpada branca fria ou lâmpada de amplo espectro, em função da dose $x_{j}$, em $\mathrm{mg} / 1$, de ácido naftaleno-acético no meio de cultura, $j=1,2,3$; é dado pela inversa da função de ligação do modelo, ou seja,

$$
\hat{\mu}_{1 j}=g^{-1}\left(\hat{\eta}_{1 j}\right)=e^{\hat{\eta}_{1 j}}+1
$$

Lembrando que, neste ponto, o nível 1 do fator tipo de radiação luminosa engloba as lâmpadas fria e de amplo espectro, a Tabela 5 apresenta as médias do número de calos produzidos por explante, dado que o explante regenerou, para os tratamentos $T_{11}, T_{12}$ e $T_{13}$, acompanhadas dos números de repetições dos tratamentos e dos valores estimados para essas médias, dados pela expressão (27).

O gráfico dos valores observados e dos valores estimados para o número médio de calos produzidos por explante de Stylosanthes scabra, dado que o explante 
regenerou, na presença de lâmpada branca fria ou de amplo espectro, em função da dose $x$, em mg/l, de ácido naftaleno-acético no meio de cultura, sendo $0,0<x<1,0$; apresenta-se na Figura 8.

Tabela 5: Médias de tratamentos do Conjunto A de dados para a variável número de calos produzidos por explante, dado que o explante regenerou, acompanhadas dos números de repetições dos tratamentos $(n)$ e de seus valores estimados $\left(\hat{\mu}_{1 j}\right)$.

\begin{tabular}{crcc}
\hline Tratamento & $n$ & Média & $\hat{\mu}_{1 j}$ \\
\hline 11 & 29 & 9,52 & 9,49 \\
12 & 9 & 1,89 & 2,05 \\
13 & 3 & 1,33 & 1,13 \\
\hline
\end{tabular}

Para o nível escuro do fator tipo de radiação luminosa, a regressão linear em função da dose de ANA não foi efetuada, pois, sob este modelo, o nível escuro só está presente em combinação com a dose $0,0 \mathrm{mg} / \mathrm{l}$ de ANA.

Uma vez que as conclusões obtidas sob o modelo Poisson truncado referem-se ao número de calos produzidos por explante, dado que o explante regenerou, realizou-se, em seguida, o ajuste do modelo binomial à proporção, $p$, de explantes que regeneraram, apresentada na Tabela 6 , para se verificar, também, o efeito das fontes de variação do modelo sobre o número de explantes que regeneram. Este ajuste foi feito no GLIM considerando-se a função de ligação canônica

$$
\eta=\theta=\ln \left(\frac{\mu}{1-\mu}\right),
$$

ou seja, a ligação logística, e o preditor linear

$$
\eta_{i j}=m+l_{i}+d_{j}+l d_{i j},
$$

onde $\eta_{i j}$ é o preditor linear correspondente à observação $p_{i j}$; 


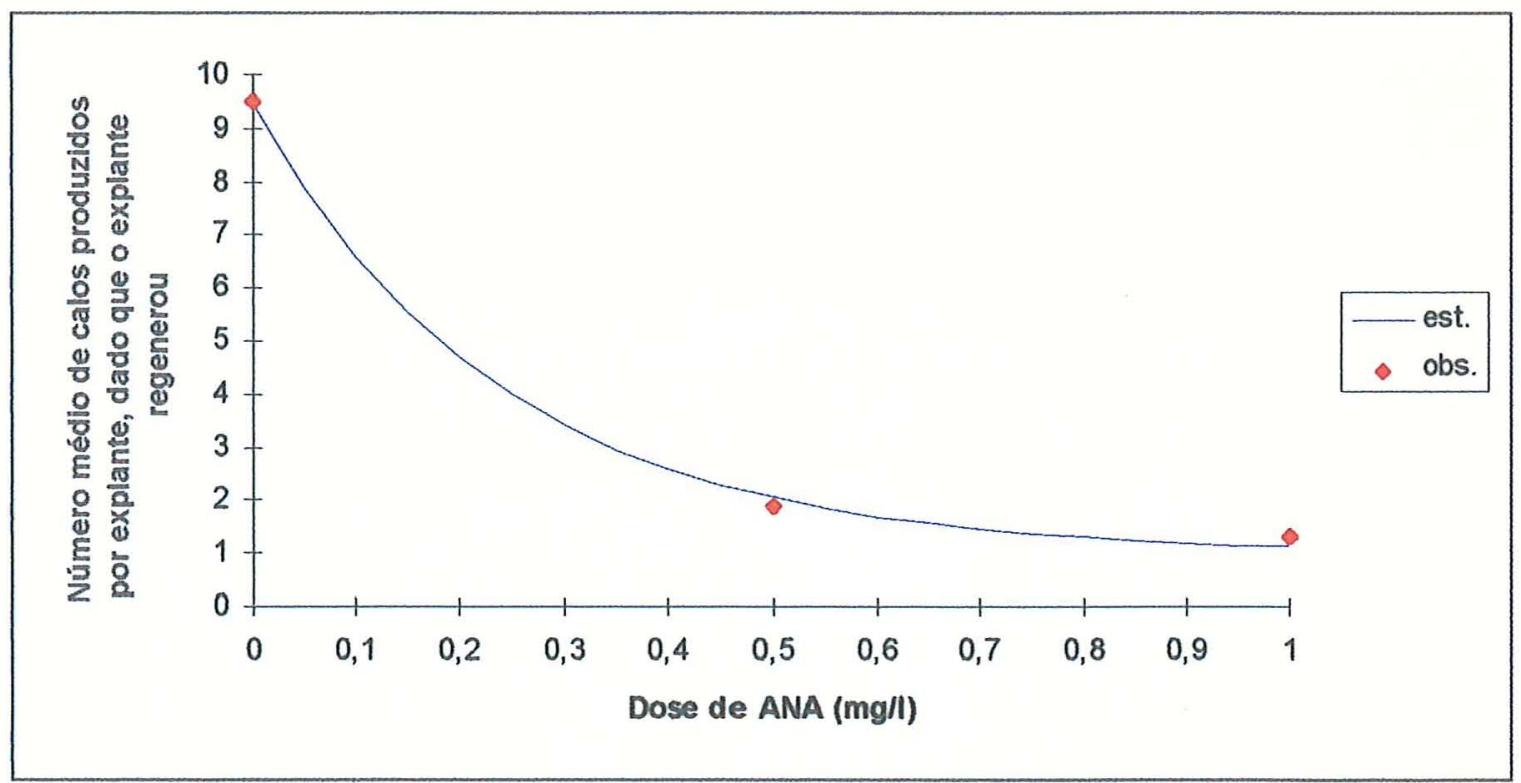

Figura 8: Gráfico dos valores observados e dos valores estimados para o número médio de calos produzidos por explante de Stylosanthes scabra, dado que o explante regenerou, na presença de lâmpada branca fria ou lâmpada de amplo espectro, em função da dose de ácido naftaleno-acético no meio de cultura. 
$m$ é uma constante inerente a todos os preditores lineares;

$l_{i}$ é o efeito do nível $i$ do fator tipo de radiação luminosa, $i=1,2,3$;

$d_{j}$ é o efeito da dose $j$ do fator ANA, $j=1,2,3$;

$l d_{i j}$ é o efeito da interação do nível $i$ do fator tipo de radiação luminosa com a dose $j$ do fator ANA.

Tabela 6: Número de explantes regenerados $(r)$, número total de explantes $(n)$ e proporção de explantes regenera$\operatorname{dos}(p=r / n)$, para os tratamentos do Conjunto A de dados.

\begin{tabular}{cccc}
\hline Tratamento & $r$ & $n$ & $p$ \\
\hline 11 & 14 & 14 & 1,00 \\
12 & 7 & 14 & 0,50 \\
13 & 2 & 14 & 0,14 \\
21 & 15 & 15 & 1,00 \\
22 & 2 & 14 & 0,14 \\
23 & 1 & 14 & 0,07 \\
31 & 3 & 14 & 0,21 \\
32 & 0 & 14 & 0,00 \\
33 & 0 & 14 & 0,00 \\
\hline
\end{tabular}

Na Tabela 7, apresentam-se os resultados da análise de "deviance" obtida no ajuste deste modelo, e o programa em GLIM utilizado para efetuar este ajuste apresenta-se no Apêndice 6 .

Uma vez que para este conjunto de dados não se têm repetições quando se considera a variável proporção de explantes regenerados, os testes para os efeitos das fontes de variação do modelo foram feitos comparando-se sua "deviance" média com o valor crítico $\chi_{[\alpha ; v)]}^{2}$, onde $\alpha$ é o nível de significância adotado e $v$ é o número de graus de liberdade associado à fonte de variação considerada. 
Tabela 7: Resultados da análise de "deviance" para a variável proporção de explantes regenerados, do Conjunto A de dados, usando o modelo binomial.

\begin{tabular}{lcc}
\hline Fontes de Variação & GL & "Deviance" \\
\hline R1 + R2) vs R3 & 1 & $24,47^{* *}$ \\
R1 vs R2 & 1 & $1,42^{n s}$ \\
(Radiação) & $(2)$ & $\left(25,89^{* *}\right)$ \\
Linear & 1 & $64,68^{* *}$ \\
Quadrático & 1 & $8,36^{* *}$ \\
(ANA) & $(2)$ & $\left(73,04^{* *}\right)$ \\
R $\times$ A & 4 & $0,53^{n s}$ \\
\hline Total & 8 & 99,46 \\
\hline & & \\
\multicolumn{1}{c}{$\chi_{(0,05 ; 1)}^{2}=3,84$} & $\chi_{(0,05 ; 2)}^{2}=5,99$ & $\chi_{(0,05 ; 4)}^{2}=9,49$ \\
$\chi_{(0,01 ; 1)}^{2}=6,64$ & $\chi_{(0,01 ; 2)}^{2}=9,22$ & $\chi_{(0,01 ; 4)}^{2}=13,29$
\end{tabular}

De acordo com os resultados apresentados na Tabela 7, tem-se que o efeito de interação entre os fatores estudados não foi significativo ao nível de $5 \%$ de probabilidade. No entanto, os gráficos que mostram o comportamento da proporção de explantes regenerados em função da dose de ANA, em cada nível do fator tipo de radiação luminosa, e o comportamento da proporção de explantes regenerados em função do tipo de radiação luminosa, em cada dose de ANA, apresentados nas Figuras 9 e 10, sugerem a existência de efeito de interação entre os dois fatores. A discordância entre a indicação dos gráficos e o resultado da análise de "deviance" explica-se pela baixa precisão dos testes, decorrente da ausência de repetições para os tratamentos. Em virtude disto, não se prosseguiu com a análise para a proporção de explantes que regeneraram, ficando, no entanto, o indicativo da existência de efeito de interação entre os fatores estudados sobre a proporção de explantes que regeneram, o que pode consistir no objeto de um novo estudo, dependendo do interesse 


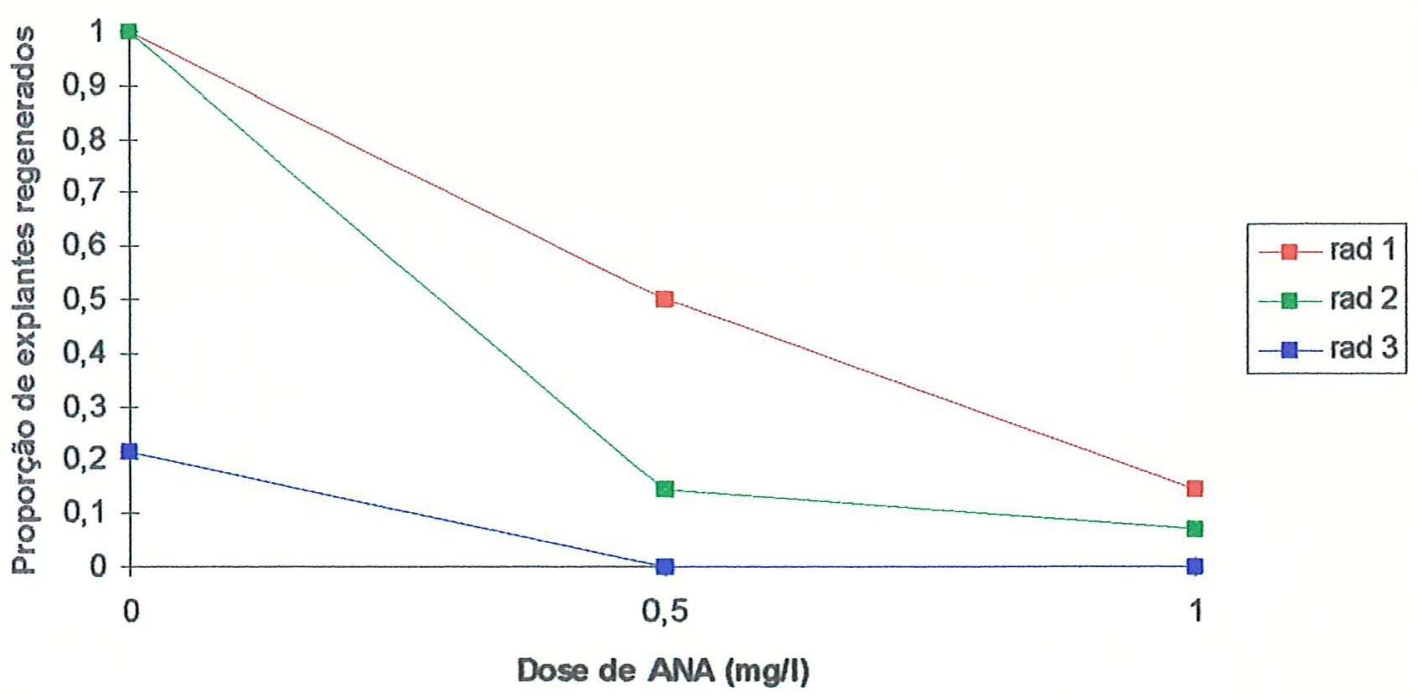

Figura 9: Gráfico da proporção de explantes regenerados versus dose de ácido naftaleno-acético no meio de cultura, em cada nível do fator tipo de radiação luminosa, para os tratamentos do Conjunto $\mathrm{A}$ de dados.

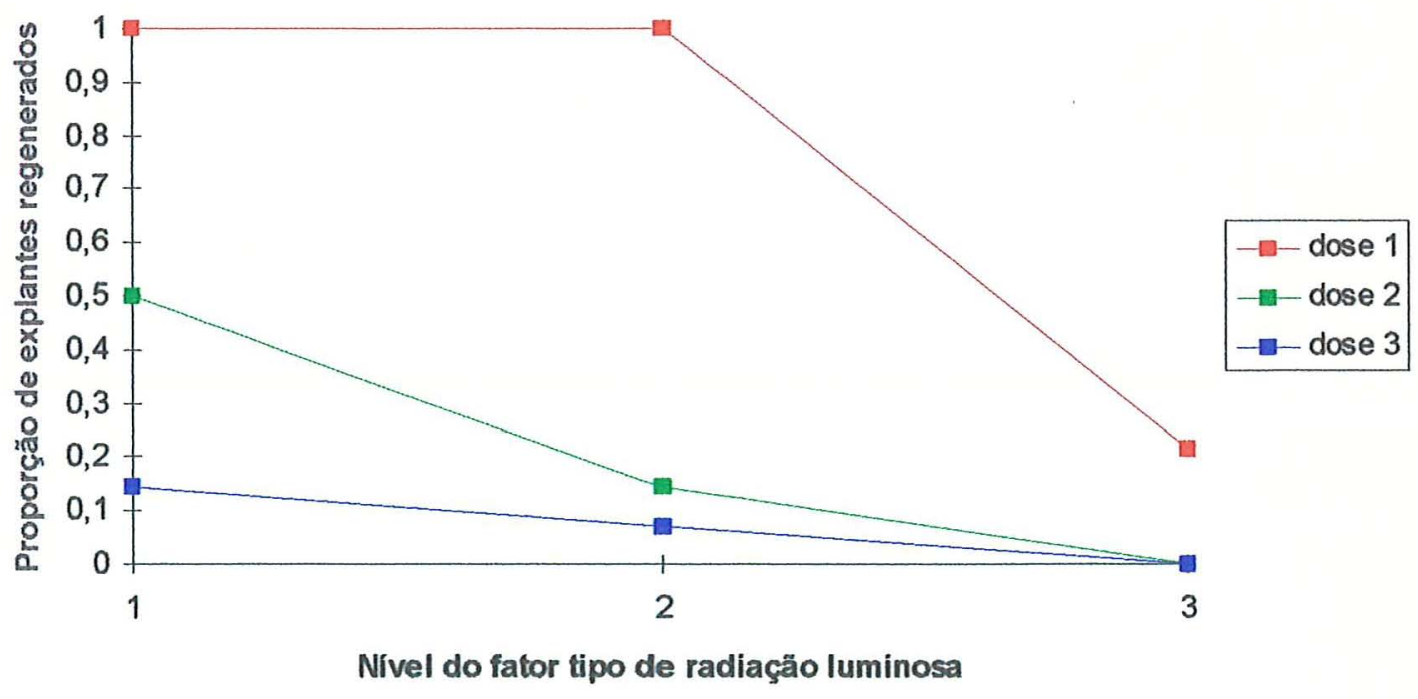

Figura 10: Gráfico da proporção de explantes regenerados versus tipo de radiação luminosa, em cada dose de ácido naftaleno-acético, para os tratamentos do Conjunto A de dados. 
do pesquisador.

\section{Conjunto B}

O resultado do teste do cociente variância-média, apresentado na Tabela 8 , indica que para 12 dentre os 14 tratamentos deste conjunto de dados rejeita-se a hipótese de que as observações seguem a distribuição de Poisson. A hipótese favorecida com a rejeição de $H_{0}$ é a de que os tratamentos têm variância superior à média, o que sugere a ocorrência de superdispersão.

Em conformidade com este resultado, o teste para detectar superdispersão foi significativo ao nível de $5 \%$ de probabilidade, o que indica a ocorrência de variação extra-Poisson neste conjunto de dados. $\mathrm{O}$ valor calculado para a estatística $T_{a}$ do teste e o valor crítico da distribuição normal padrão para $\alpha=0,05$ são:

$$
T_{a}=83,43^{*} \quad Z_{0,05}=1,64 .
$$

Este resultado foi confirmado ao se ajustar o modelo Poisson às observações. Neste caso, os resultados obtidos na análise de "deviance", apresentados na Tabela 9, mostram que o teste de $\chi^{2}$ para a "deviance" residual do modelo, associada a 266 graus de liberdade, foi significativo ao nfvel de $1 \%$ de probabilidade. Isto indica que o ajuste não foi bom e confirma a ocorrência de variação extra-Poisson indicada pelo teste para superdispersão.

A pobreza do ajuste vem a ser evidenciada pela análise dos gráficos apresentados nas Figuras 11, 12, 13 e 14.

Examinando-se os gráficos dos componentes de "deviance" padronizados versus índices e dos componentes de "deviance" estudentizados externamente versus índices, que constam das Figuras 11 e 12, verifica-se que os resíduos assumem valores desde -6 até +7 , os quais deveriam variar entre -2 e +2 se o modelo fosse correto.

O gráfico dos componentes de "deviance" padronizados versus tratamentos ordenados pelas médias apresentado na Figura 13, por sua vez, mostra uma 
Tabela 8: Teste do cociente variância-média aplicado aos tratamentos do Conjunto B de dados.

\begin{tabular}{ccrrr}
\hline Tratamento & $n$ & \multicolumn{1}{c}{$\bar{y}$} & \multicolumn{1}{c}{$s^{2}$} & $I(n-1)$ \\
\hline 11 & 20 & 4,30 & 9,69 & $42,84^{*}$ \\
12 & 20 & 23,90 & 105,57 & $83,92^{*}$ \\
13 & 20 & 39,75 & 799,78 & $382,28^{*}$ \\
14 & 20 & 35,50 & 482,68 & $258,34^{*}$ \\
15 & 20 & 11,75 & 150,30 & $243,04^{*}$ \\
16 & 20 & 14,65 & 160,34 & $207,96^{*}$ \\
17 & 20 & 17,05 & 215,21 & $239,82^{*}$ \\
21 & 20 & 0,90 & 1,67 & $35,33^{*}$ \\
22 & 20 & 0,90 & 1,67 & $35,33^{*}$ \\
23 & 20 & 0,45 & 0,89 & $37,67^{*}$ \\
24 & 20 & 0,20 & 0,27 & $26,00^{n s}$ \\
25 & 20 & 0,15 & 0,13 & $17,00^{n s}$ \\
26 & 20 & 0,80 & 2,90 & $69,00^{*}$ \\
27 & 20 & 0,40 & 0,57 & $27,00^{n s}$ \\
\hline & & & &
\end{tabular}


Tabela 9: Resultados da análise de "deviance" para a variável número de calos produzidos por explante, do Conjunto B de dados, usando o modelo Poisson.

\begin{tabular}{lcccc}
\hline Fontes de Variação & GL & "Deviance" & "Deviance" média & Razão \\
\hline Carvão (C) & 1 & 3469,00 & 3469,00 & $548,02^{* *}$ \\
$\mathrm{~T}^{\dagger}$ vs (AC + TIBA) & 1 & 397,20 & 397,20 & $62,75^{* *}$ \\
AC vs TIBA & 1 & 440,80 & 440,80 & $69,64^{* *}$ \\
Níveis de AC & 2 & 77,70 & 38,85 & $6,14^{* *}$ \\
Níveis de TIBA & 2 & 21,60 & 10,80 & $1,71^{n s}$ \\
(Suplemento) & $(6)$ & $(937,30)$ & & \\
C x [T vs (AC + TIBA)] & 1 & 44,60 & 44,60 & $7,04^{* *}$ \\
C x [AC vs TIBA] & 1 & 6,40 & 6,40 & $1,01^{n s}$ \\
C x níveis de AC & 2 & 17,30 & 8,65 & $1,37^{n s}$ \\
C x níveis de TIBA & 2 & 8,00 & 4,00 & $0,63^{n s}$ \\
(C x Suplemento) & $(6)$ & $(76,30)$ & & \\
Resíduo & 266 & $1685,30^{* *}$ & 6,33 & \\
\hline Total & 279 & 6167,90 & & \\
\hline
\end{tabular}

$$
\begin{array}{ll}
\chi_{(0,05 ; 266)}^{2}=305,55 & \chi_{(0,01 ; 266)}^{2}=324,94 \\
F_{(0,05 ; 1,266)}=3,88 & F_{(0,05 ; 2,266)}=3,03 \\
F_{(0,01 ; 1,266)}=6,73 & F_{(0,01 ; 2,266)}=4,68
\end{array}
$$

${ }^{\dagger} \mathbf{T}=$ Testemunha

${ }^{\ddagger} \mathrm{AC}=$ Água de coco 


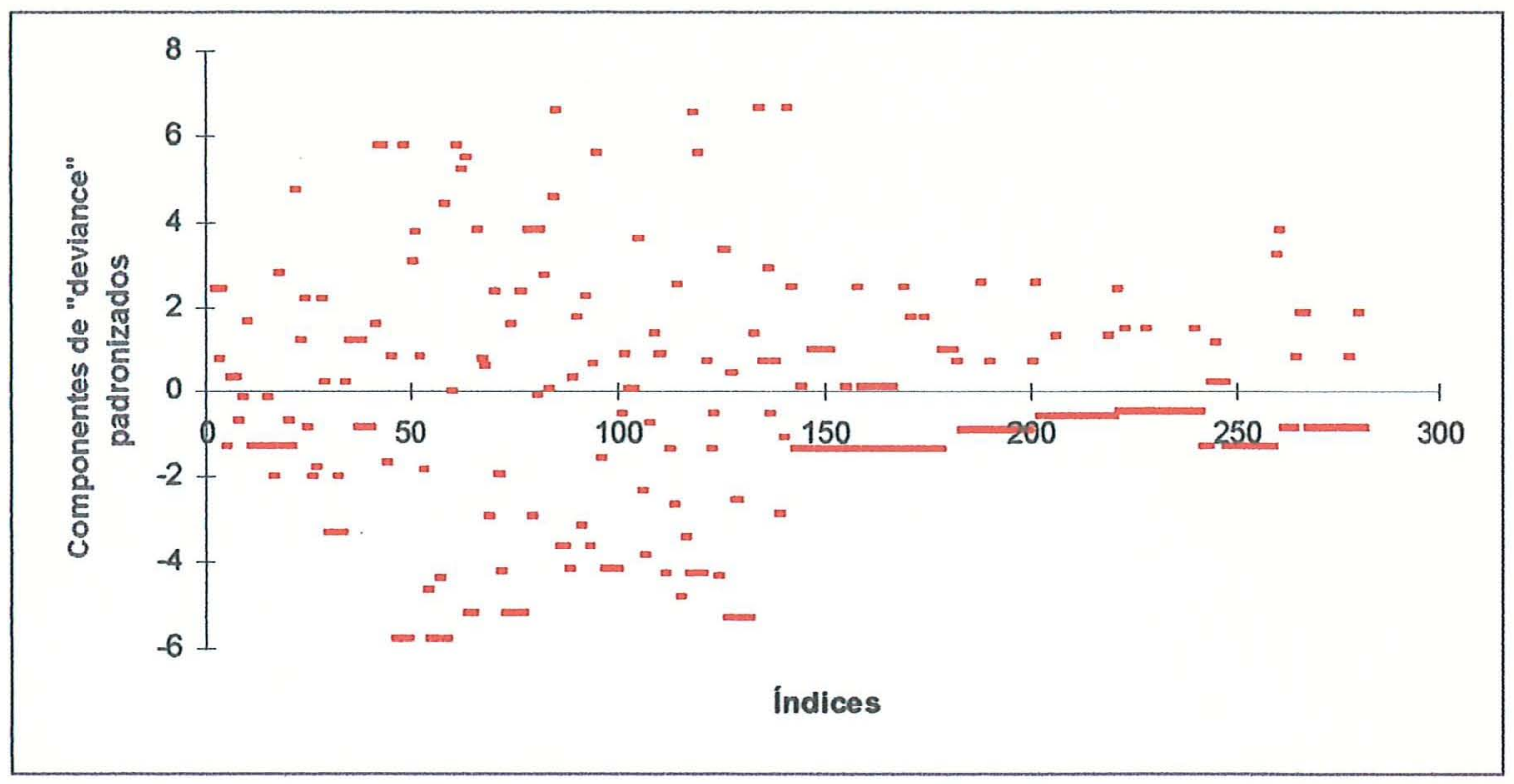

Figura 11: Gráfico dos componentes de "deviance" padronizados versus índices, resultante do ajuste do modelo Poisson ao Conjunto B de dados.

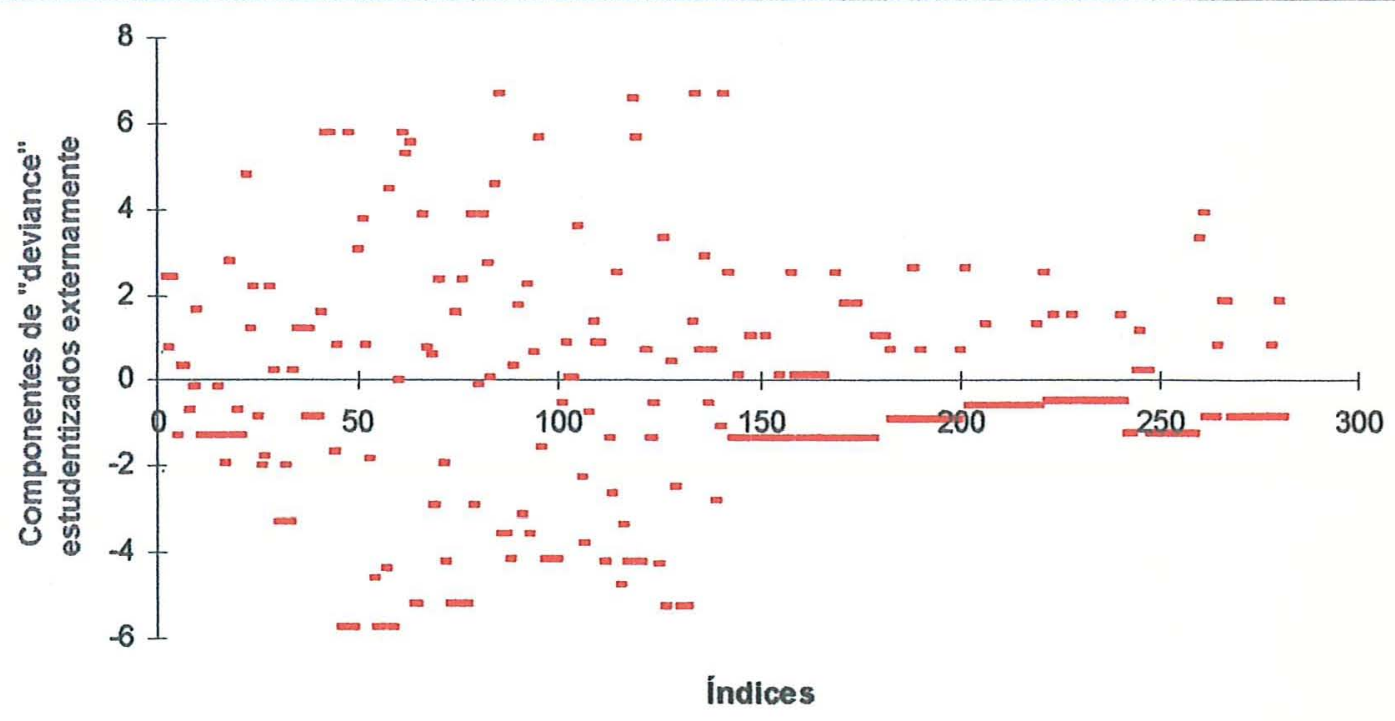

Figura 12: Gráfico dos componentes de "deviance" estudentizados externamente versus índices, resultante do ajuste do modelo Poisson ao Conjunto B de dados. 


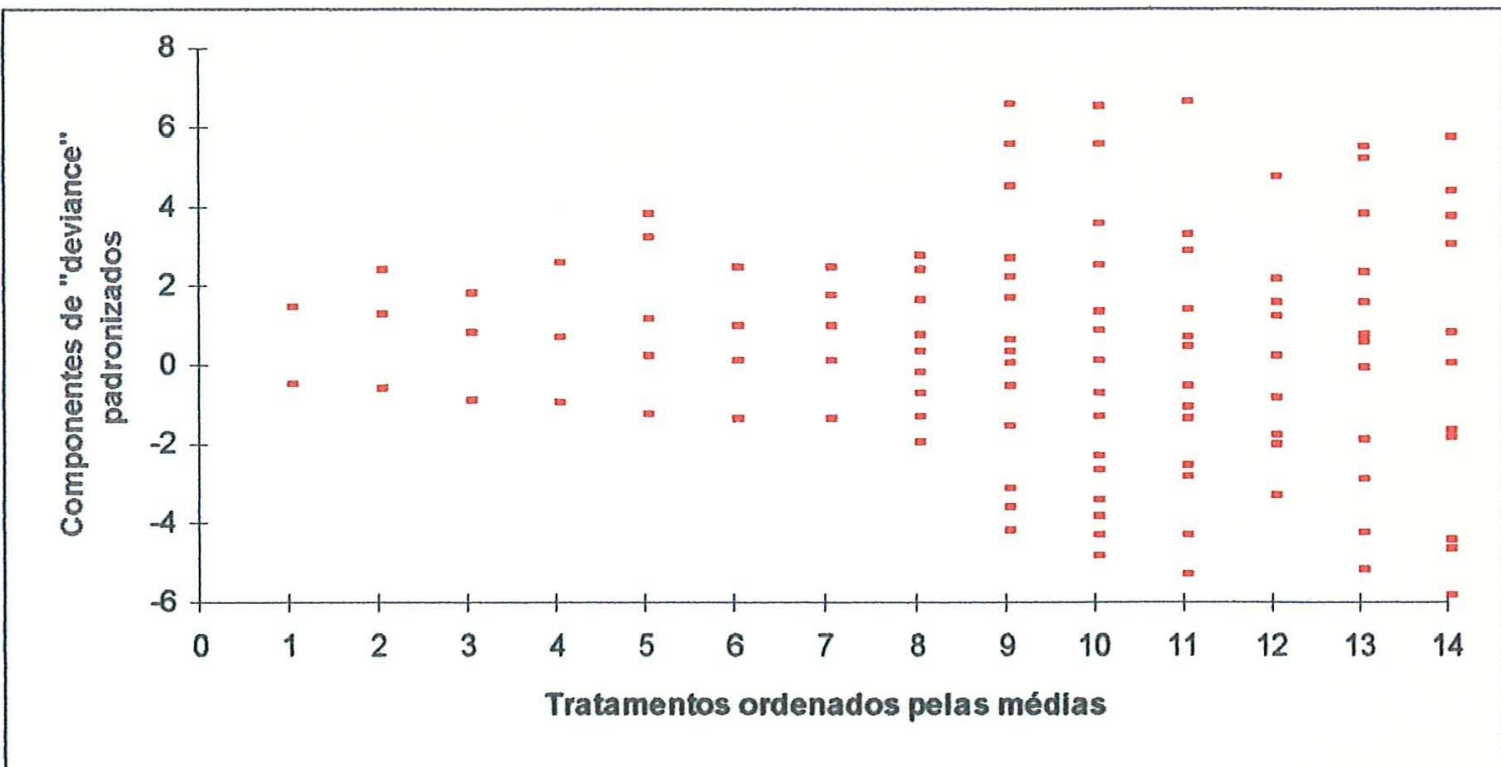

Figura 13: Gráfico dos componentes de "deviance" padronizados versus tratamentos ordenados pelas médias, resultante do ajuste do modelo Poisson ao Conjunto $\mathrm{B}$ de dados.

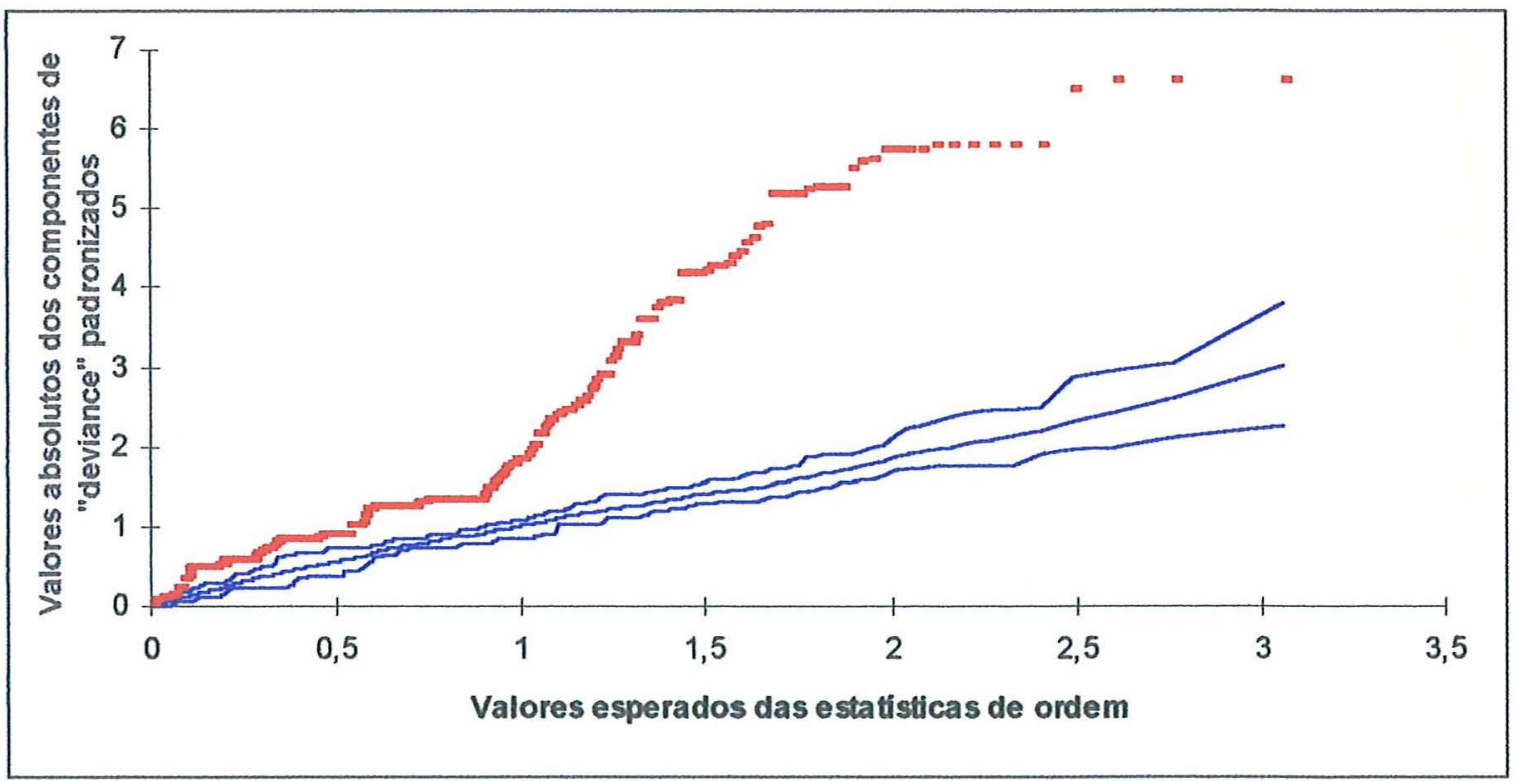

Figura 14: Gráfico normal de probabilidade para os valores absolutos dos componentes de "deviance" padronizados, resultante do ajuste do modelo Poisson ao Conjunto B de dados. 
tendência de crescimento da variância dos resíduos com o crescimento da média, indicando heterogeneidade de variâncias, ou seja, que o modelo não está explicando alguma fonte de variação das observações. Na Tabela 21, que consta do Apêndice 14, apresentam-se os tratamentos ordenados pelas médias.

O gráfico normal de probabilidade para os valores absolutos do componentes de "deviance" padronizados com envelope simulado, apresentado na Figura 14, mostra que o modelo está subestimando as observações, uma vez que os valores dos resíduos apresentam-se muito acima da linha superior do envelope simulado.

Por outro lado, considerando-se o modelo Poisson truncado para este conjunto de dados, obteve-se que o teste de $\chi^{2}$ para a "deviance" residual do modelo, associada a 165 graus de liberdade, foi significativo ao nível de $1 \%$ de probabilidade, conforme se observa na Tabela 10, que apresenta os resultados obtidos na análise de "deviance". Isto indica que o ajuste não foi bom.

A pobreza do ajuste é confirmada pela análise dos gráficos que se apresentam nas Figuras 15, 16 e 17. Com relação a esses gráficos, as mesmas considerações feitas para o modelo Poisson são válidas para este modelo, de forma que, praticamente, não se obteve nenhuma melhora no ajuste com o modelo Poisson truncado.

Considerando-se, alternativamente, o modelo binomial negativo para este conjunto de dados, obteve-se, inicialmente, o valor adotado para o parâmetro $k$, conforme o exposto no item 3.2.2.3. Ou seja, calculando-se a "deviance" residual do modelo para 50 valores diferentes de $k$, os quais partiram de 0,10 e foram acrescidos sucessivamente de 0,05 , chegando a 2,55 , obteve-se que o valor que resultou na "deviance" residual mais próxima do número de graus de liberdade a ela associado foi $k=1,45$, o qual foi aproximado para $k=1,50$. Os 50 valores atribuídos a $k$ e as respectivas "deviances" residuais do modelo apresentam-se na Tabela 22, que consta do Apêndice 15.

Ajustando-se, então, o modelo binomial negativo com parâmetro $k=1,5$ a este conjunto de dados, os resultados obtidos na análise de "deviance" 
apresentam-se na Tabela 11.

Tabela 10: Resultados da análise de "deviance" para a variável número de calos produzidos por explante, do Conjunto B de dados, usando o modelo Poisson truncado.

\begin{tabular}{|c|c|c|c|c|}
\hline Fontes de Variação & GL & "Deviance" & "Deviance" média & Razão \\
\hline Carvão (C) & 1 & 981,00 & 981,00 & $103,70^{* *}$ \\
\hline $\mathrm{T}$ vs $(\mathrm{AC}+\mathrm{TIBA})$ & 1 & 421,80 & 421,80 & $44,59^{* *}$ \\
\hline $\mathrm{AC}$ vs TIBA & 1 & 442,80 & 442,80 & $46,81^{* *}$ \\
\hline Níveis de AC & 2 & 82,57 & 41,28 & $4,36^{*}$ \\
\hline Niveis de TIBA & 2 & 20,23 & 10,11 & $1,07^{n s}$ \\
\hline (Suplemento) & $(6)$ & $(967,40)$ & & \\
\hline $\mathrm{C} \times[\mathrm{T}$ vs $(\mathrm{AC}+\mathrm{TIBA})]$ & 1 & 15,90 & 15,90 & $1,68^{n s}$ \\
\hline $\mathrm{C} \times[\mathrm{AC}$ vs TIBA $]$ & 1 & 4,13 & 4,13 & $0,44^{n s}$ \\
\hline $\mathrm{C} \times$ nfveis de $\mathrm{AC}$ & 2 & 4,64 & 2,32 & $0,24^{n s}$ \\
\hline $\mathrm{C} \times$ níveis de TIBA & 2 & 7,21 & 3,60 & $0,38^{n s}$ \\
\hline (C x Suplemento) & (6) & $(31,88)$ & & \\
\hline Resíduo & 165 & $1560,32^{* *}$ & 6,30 & \\
\hline Total & 178 & 3540,60 & & \\
\hline \multicolumn{2}{|c|}{$\chi_{(0,05 ; 165)}^{2}=196,21$} & \multicolumn{2}{|c|}{$\chi_{(0,01 ; 165)}^{2}=211,83$} & \\
\hline \multicolumn{2}{|c|}{$F_{(0,05 ; 1,165)}=3,90$} & \multicolumn{2}{|c|}{$F_{(0,05 ; 2,165)}=3,05$} & \\
\hline \multicolumn{2}{|c|}{$F_{(\mathbf{0 , 0 1} \mathbf{0 1}, \mathbf{1 6 5 )}}=6,79$} & \multicolumn{2}{|c|}{$F_{(0,01 ; 2,165)}=4,74$} & \\
\hline
\end{tabular}




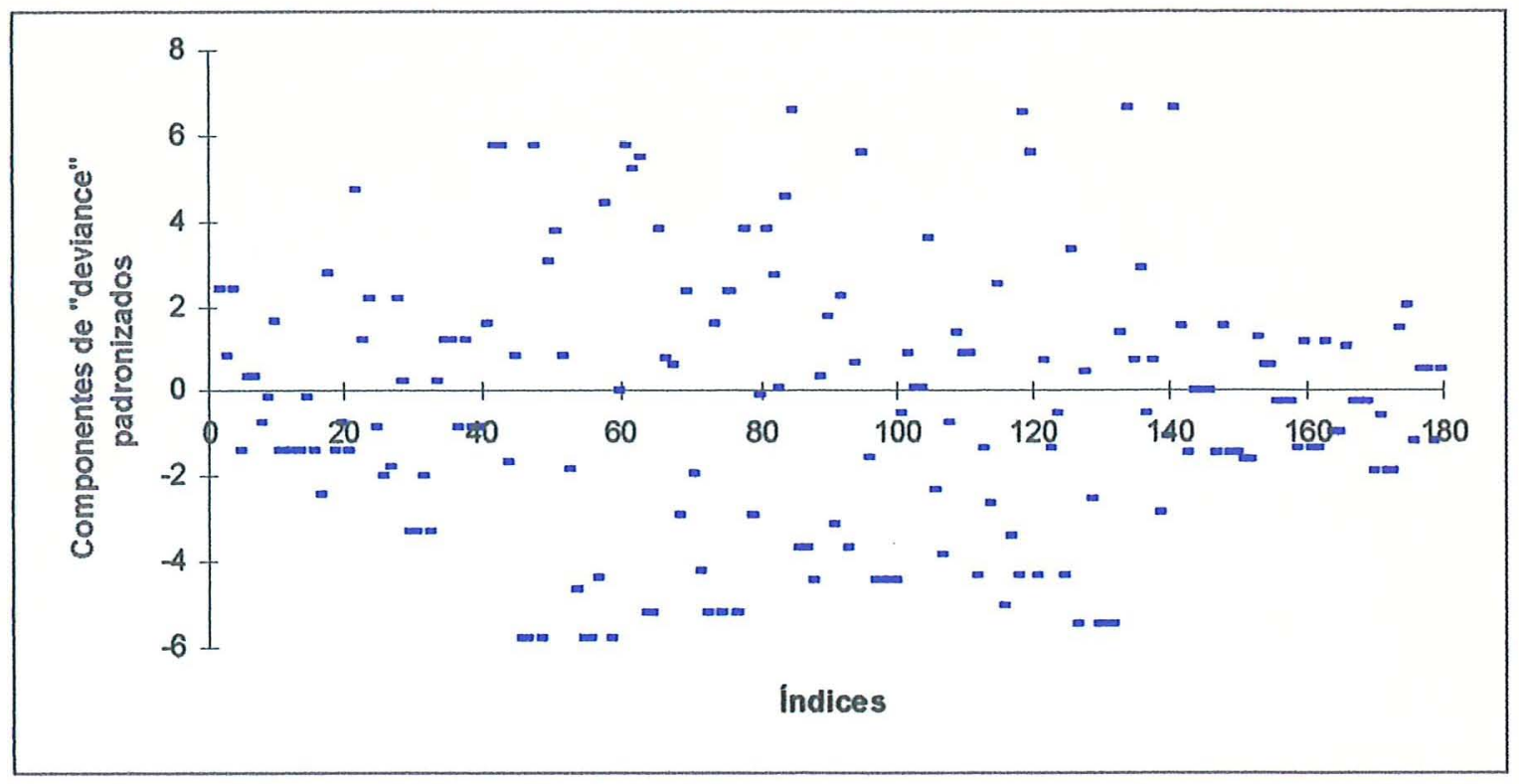

Figura 15: Gráfico dos componentes de "deviance" padronizados versus índices, resultante do ajuste do modelo Poisson truncado ao Conjunto B de dados.

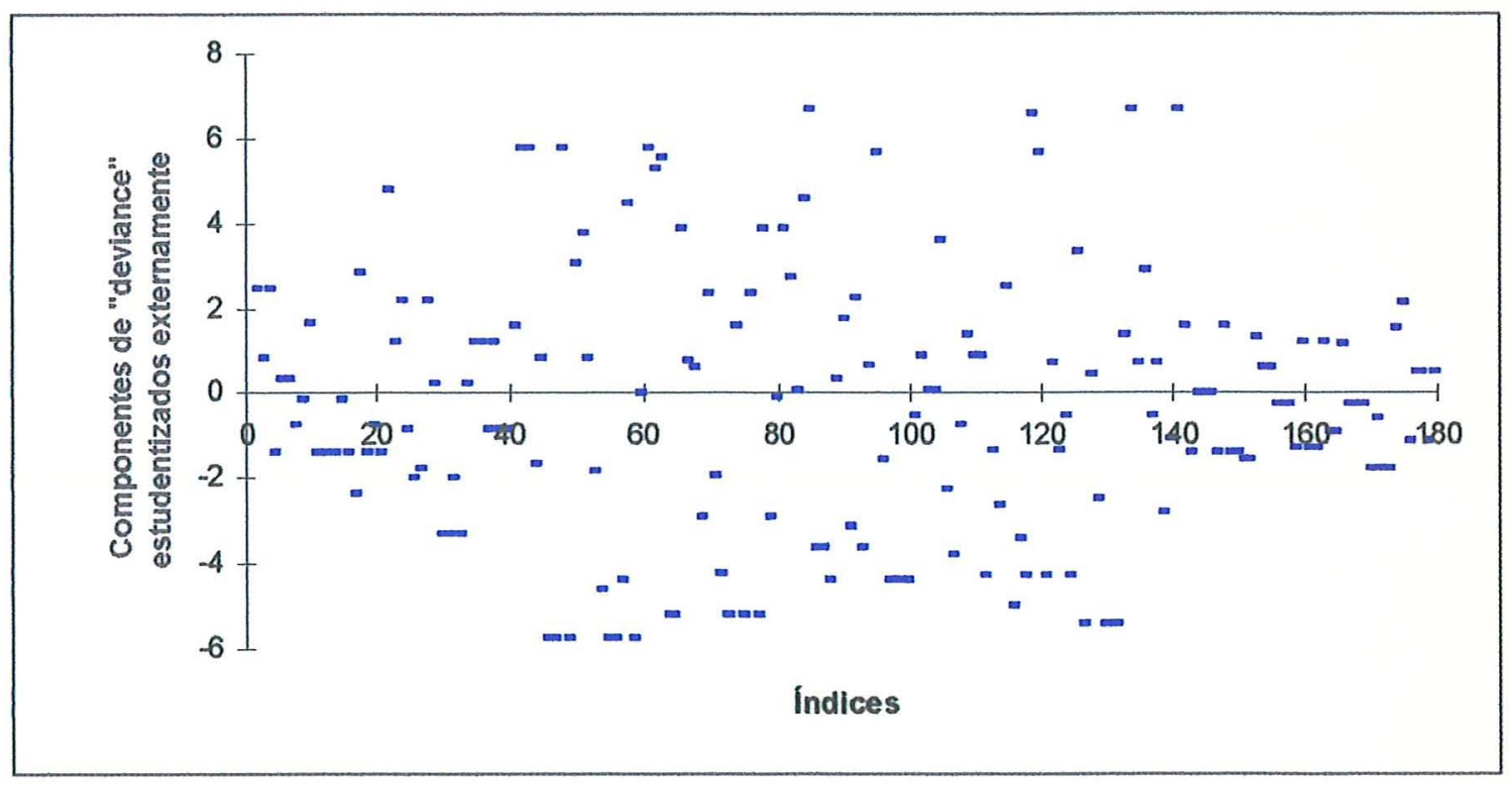

Figura 16: Gráfico dos componentes de "deviance" estudentizados externamente versus índices, resultante do ajuste do modelo Poisson truncado ao Conjunto B de dados. 


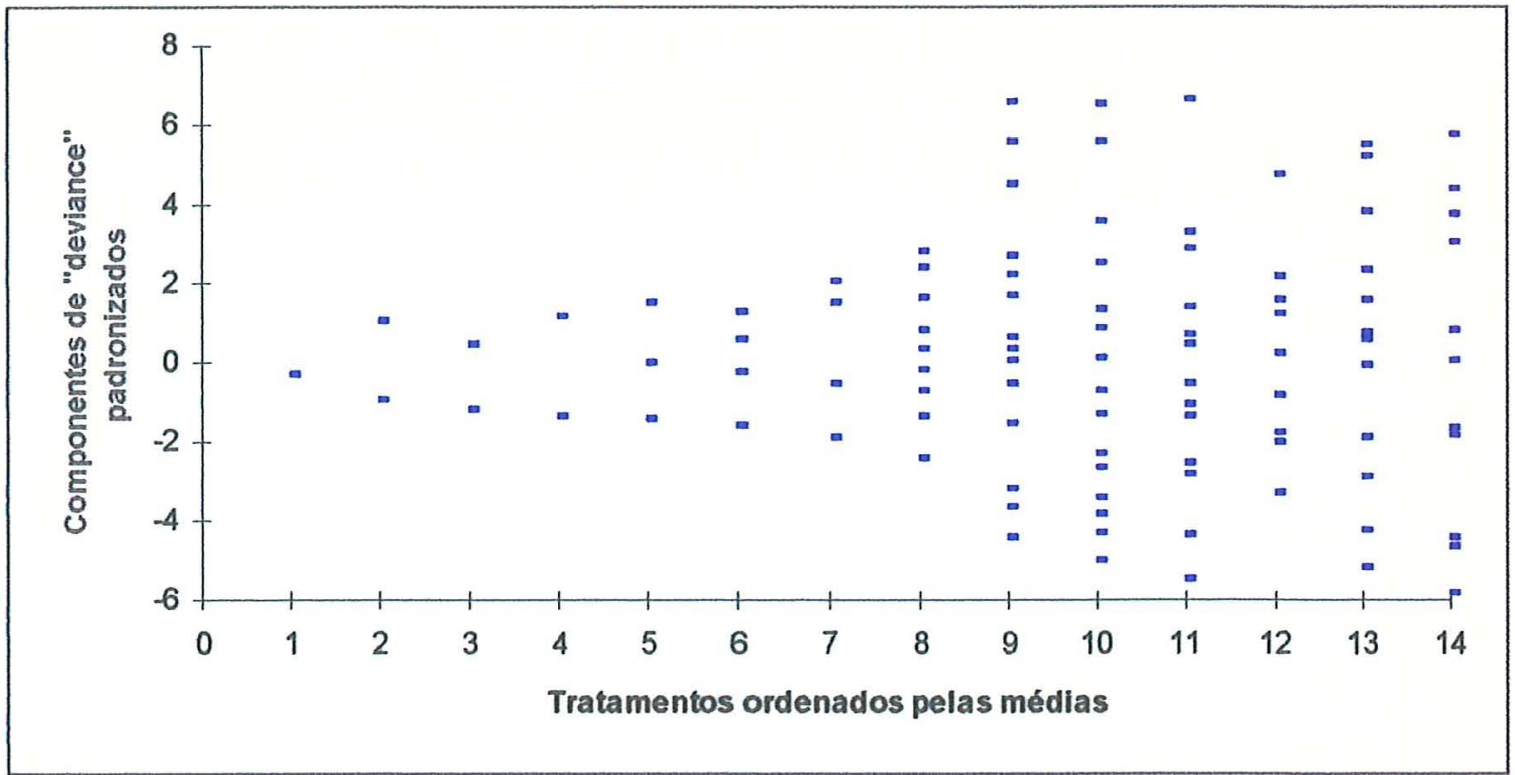

Figura 17: Gráfico dos componentes de "deviance" padronizados versus tratamentos ordenados pelas médias, resultante do ajuste do modelo Poisson truncado ao Conjunto $\mathrm{B}$ de dados. 
Tabela 11: Resultados da análise de "deviance" para a variável número de calos produzidos por explante, do Conjunto B de dados, usando o modelo binomial negativo.

\begin{tabular}{|c|c|c|c|c|}
\hline Fontes de Variação & GL & "Deviance" & "Deviance" média & Razão \\
\hline Carvão (C) & 1 & 677,47 & 677,47 & $664,19^{* *}$ \\
\hline $\mathrm{T}$ vs $(\mathrm{AC}+\mathrm{TIBA})$ & 1 & 40,63 & 40,63 & $39,83^{* *}$ \\
\hline AC vs TIBA & 1 & 28,05 & 28,05 & $27,50^{* *}$ \\
\hline Niveis de AC & 2 & 3,59 & 1,79 & $1,75^{n s}$ \\
\hline Níveis de TIBA & 2 & 2,14 & 1,07 & $1,05^{n s}$ \\
\hline (Suplemento) & (6) & $(74,41)$ & & \\
\hline $\mathrm{C} \times[\mathrm{T}$ vs $(\mathrm{AC}+\mathrm{TIBA})]$ & 1 & 7,13 & 7,13 & $6,99^{* *}$ \\
\hline $\mathrm{C} \times[\mathrm{AC}$ vs TIBA $]$ & 1 & 0,05 & 0,05 & $0,05^{n s}$ \\
\hline $\mathrm{C} x$ niveis de $\mathrm{AC}$ & 2 & 7,74 & 3,87 & $3,79^{*}$ \\
\hline $\mathrm{C} \times$ níveis de TIBA & 2 & 7,48 & 3,74 & $3,67^{*}$ \\
\hline (C $\times$ Suplemento $)$ & (6) & $(22,40)$ & & \\
\hline Resíduo & 266 & $271,02^{\text {ns }}$ & 1,02 & \\
\hline Total & 279 & 1045,30 & & \\
\hline \multicolumn{2}{|c|}{$\chi_{(0,05 ; 266)}^{2}=305,55$} & \multicolumn{2}{|c|}{$\chi_{(0,01 ; 266)}^{2}=324,94$} & \\
\hline \multicolumn{2}{|c|}{$F_{(0,05 ; 1,266)}=3,88$} & \multicolumn{2}{|c|}{$F_{(0,05 ; 2,266)}=3,03$} & \\
\hline \multicolumn{2}{|c|}{$F_{(0,01 ; 1,266)}=6,73$} & \multicolumn{2}{|c|}{$F_{(0,01 ; 2,266)}=4,68$} & \\
\hline
\end{tabular}

O teste de $\chi^{2}$ para a "deviance" residual do modelo, associada a 266 graus de liberdade, não foi significativo ao nível de $5 \%$ de probabilidade, o que, tendo em vista o critério adotado para a obtenção do valor de $k$ considerado no modelo, já era conhecido.

Analisando-se os gráficos dos componentes de "deviance" padronizados versus índices e dos componentes de "deviance" estudentizados externamen- 
te versus índices, apresentados nas Figuras 18 e 19, verifica-se que os mesmos apresentam-se relativamente próximos de seu padrão ideal, com alguns pontos distanciados dos demais abaixo e acima das retas de ordenadas iguais a -2 e +2 , respectivamente.

O gráfico dos componentes de "deviance" padronizados versus tratamentos ordenados pelas médias apresentado na Figura 20, por sua vez, revela uma leve tendência linear, ou seja, à medida que a média dos tratamentos aumenta, o intervalo dos valores assumidos pelos respectivos resíduos desloca-se para o sentido negativo do eixo das ordenadas.

A análise gráfica mostra que o modelo binomial negativo teve um ajuste razoavelmente bom.

Considerando-se os três modelos ajustados a este conjunto de dados, verifica-se que o modelo binomial negativo proporcionou uma melhora considerável na qualidade do ajuste em relação aos modelos Poisson e Poisson trundado. Nestes, além dos resíduos assumirem valores entre $-6 \mathrm{e}+7$, é bem evidente o padrão de crescimento da variância com o crescimento da média mostrado pelo gráfico dos componentes de "deviance" padronizados versus tratamentos ordenados pelas médias.

No entanto, levando-se em conta que os gráficos resultantes do ajuste do modelo binomial negativo não satisfazem plenamente seus padrões ideais, podese admitir a obtenção de um ajuste melhor. Uma alternativa seria um modelos mais sofisticado, em que o parâmetro $k$ da distribuição binomial negativa é considerado desconhecido e estimado com os demais parâmetros desconhecidos do modelo. Podese considerar, neste caso, o modelo proposto por BRESLOW (1984), um modelo loglinear ajustado pelo método dos mínimos quadrados reponderados iterativamente em que a variação extra-Poisson é considerada uma constante desconhecida e incluída no modelo através do peso atribuído às observações.

Quanto aos resultados do teste $\mathrm{F}$ aproximado obtidos nos três ajustes efetuados para este conjunto de dados, tem-se que, para o modelo binomial negativo, 


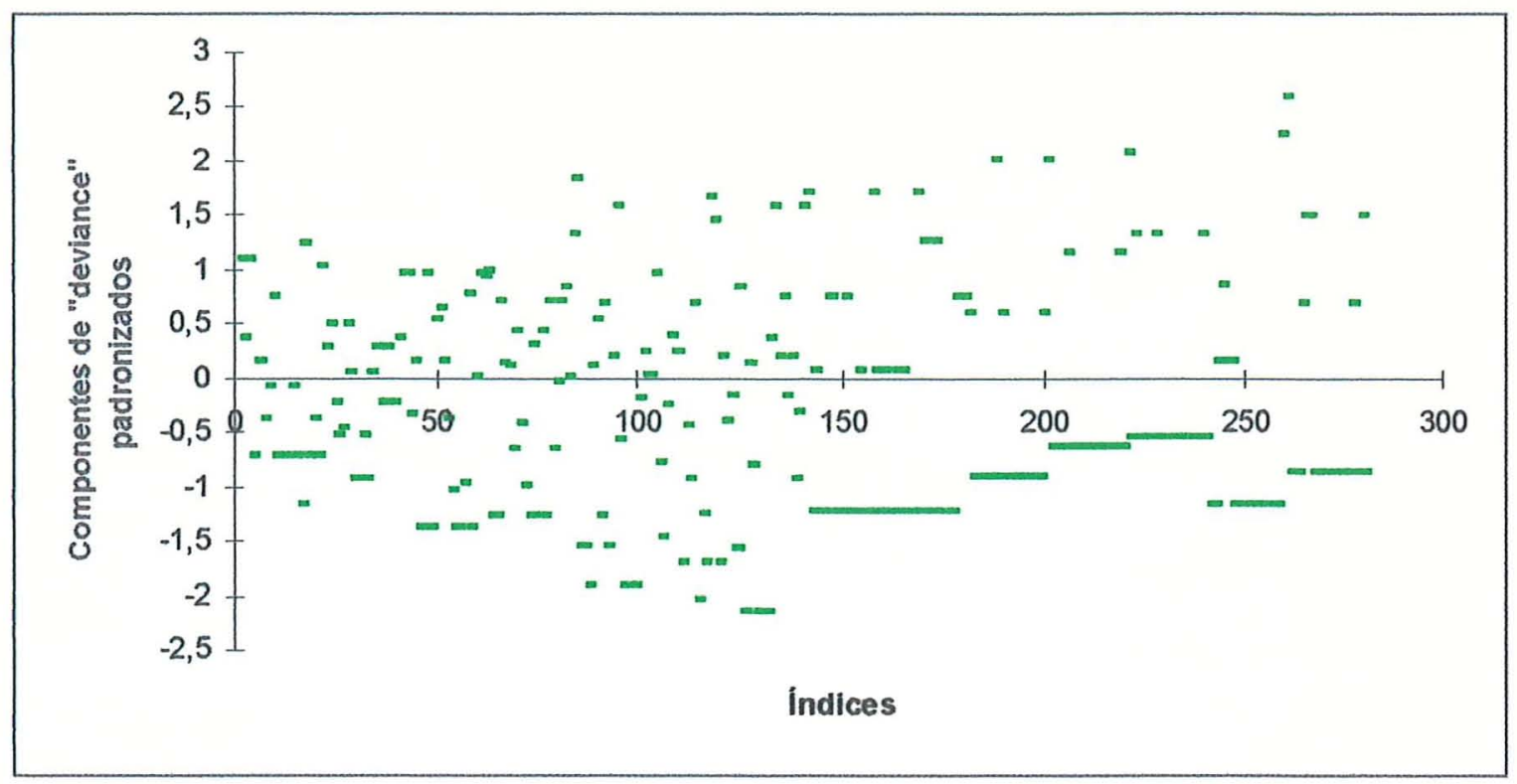

Figura 18: Gráfico dos componentes de "deviance" padronizados versus índices, resultante do ajuste do modelo binomial negativo ao Conjunto B de dados.

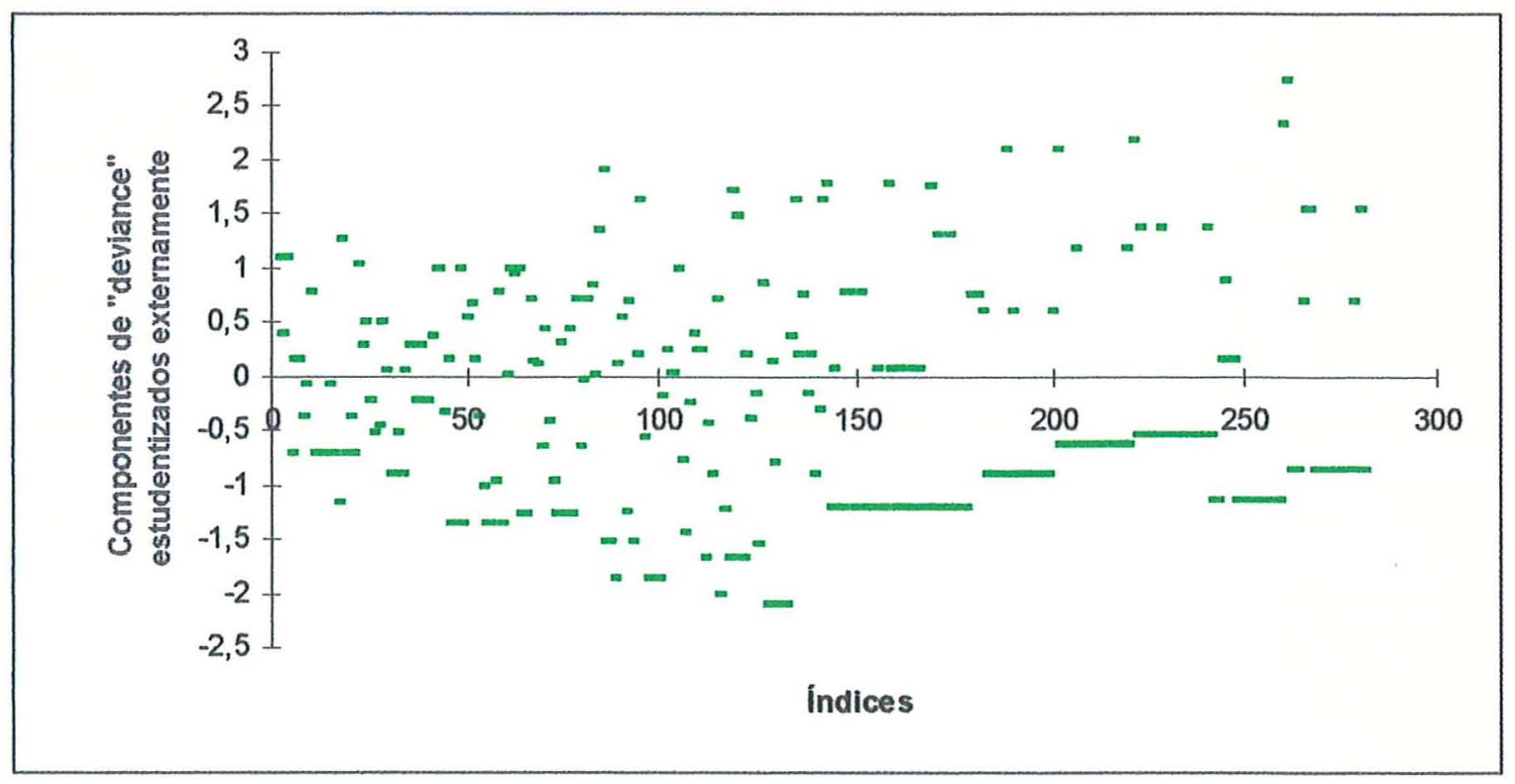

Figura 19: Gráfico dos componentes de "deviance" estudentizados externamente versus índices, resultante do ajuste do modelo binomial negativo ao Conjunto B de dados. 


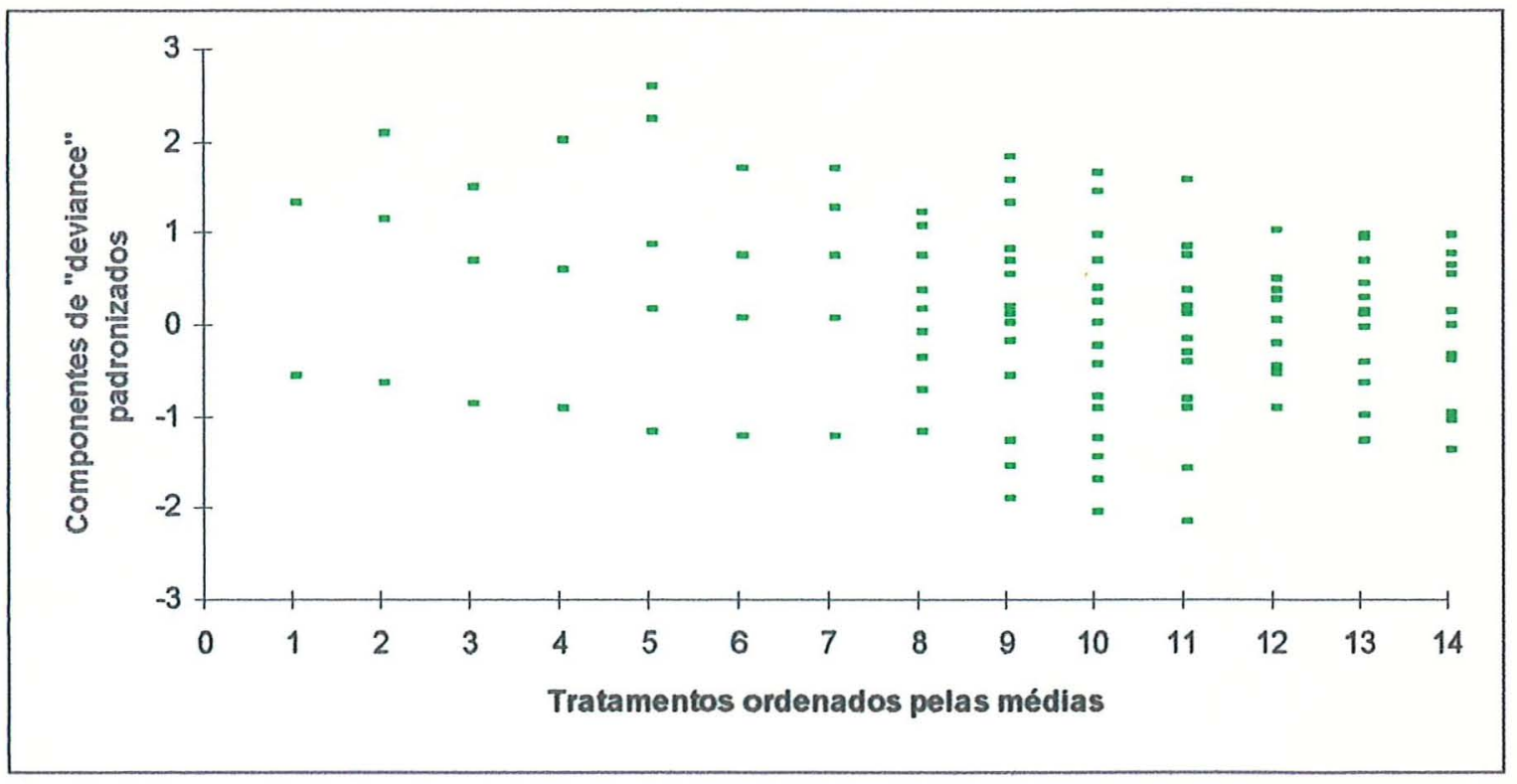

Figura 20: Gráfico dos componentes de "deviance" padronizados versus tratamentos ordenados pelas médias, resultante do ajuste do modelo binomial negativo ao Conjunto $\mathrm{B}$ de dados. 
conforme se verifica na Tabela 11, com exceção da interação carvão x [água de coco vs TIBA], todas as demais tiveram efeito significativo ( $\alpha=0,05$ e $\alpha=0,01)$. Já sob o modelo Poisson truncado, conforme se observa na Tabela 10, nenhuma interação teve efeito significativo ao nivel de $5 \%$ de probabilidade, e, sob o modelo Poisson, o teste $F$ foi significativo $(\alpha=0,01)$ somente para a interação carvão x [testemunha vs (água de coco + TIBA)], conforme se verifica na Tabela 9. Esses resultados são coerentes com aqueles dos testes para as "deviances" residuais dos modelos e com a análise gráfica, os quais indicam que os modelos Poisson e Poisson truncado não explicam todas as fontes da variação contida nos dados, de modo que suas "deviances" residuais resultam superestimadas e não permitem que se detectem efeitos significativos.

Considerando-se, então, os resultados da análise de "deviance" apresentados na Tabela 11 referentes ao modelo binomial negativo, que melhor se ajustou a este conjunto de dados, tem-se que o efeito da interação carvão $\times$ [água de coco vs TIBA] não foi significativo ao nível de $5 \%$ de probabilidade. Por sua vez, o contraste água de coco vs TIBA teve efeito significativo ao nível de $1 \%$ de probabilidade e, portanto, conclui-se que a água de coco difere de TIBA quanto ao efeito provocado no número de calos produzidos por explante de Stylosanthes scabra. Conforme se verifica na Tabela 12, que apresenta as médias dos tratamentos para a variável estudada, o número médio de calos produzidos por explante é maior na presença de água de coco do que na presença de TIBA.

Considerando-se que as demais interações tiveram efeito significativo, os efeitos principais das fontes de variação do modelo foram desdobrados com o efeito da interação em que estavam envolvidos. De acordo com o programa em GLIM apresentado no Apêndice 9, os contrastes testemunha vs [água de coco + TIBA], níveis de água de coco e níveis de TIBA foram estudados dentro dos níveis do fator carvão, e os resultados da análise de "deviance" correspondente apresentam-se na Tabela 13.

O teste $F$ aproximado foi significativo ao nível de $1 \%$ de probabilidade 
Tabela 12: Médias dos tratamentos do Conjunto B de dados para a variável número de calos produzidos por explante, acompanhadas dos números de repetições dos tratamentos $(n)$.

\begin{tabular}{ccc}
\hline Tratamento & Média & $n$ \\
\hline 11 & 4,30 & 20 \\
12 & 23,90 & 20 \\
13 & 39,75 & 20 \\
14 & 35,50 & 20 \\
15 & 11,75 & 20 \\
16 & 14,65 & 20 \\
17 & 17,05 & 20 \\
21 & 0,00 & 20 \\
22 & 0,90 & 20 \\
23 & 0,45 & 20 \\
24 & 0,20 & 20 \\
25 & 0,15 & 20 \\
26 & 0,80 & 20 \\
27 & 0,40 & 20 \\
\hline
\end{tabular}


Tabela 13: Resultados da análise de "deviance" para a variável número de calos produzidos por explante, do Conjunto B de dados, com desdobramento das interações, usando o modelo binomial negativo.

\begin{tabular}{|c|c|c|c|c|}
\hline Fontes de Variação & GL & "Deviance" & "Deviance" média & Razão \\
\hline $\mathrm{T}$ vs $(\mathrm{AC}+\mathrm{TIBA}) \mid \mathrm{C} 1$ & 1 & 44,43 & 44,43 & $43,56^{* *}$ \\
\hline $\mathrm{T}$ vs $(\mathrm{AC}+\mathrm{TIBA}) \mid \mathrm{C} 2$ & 1 & 3,33 & 3,33 & $3,26^{n s}$ \\
\hline$(\mathrm{T}$ vs $(\mathrm{AC}+\mathrm{TIBA}))$ & $(2)$ & $(47,76)$ & & \\
\hline Níveis de $\mathrm{AC} \mid \mathrm{C} 1$ & 2 & 3,89 & 1,94 & $1,90^{n s}$ \\
\hline Níveis de $\mathrm{AC} \mid \mathrm{C} 2$ & 2 & 7,44 & 3,72 & $3,65^{*}$ \\
\hline ( Niveis de $A C$ ) & (4) & $(11,33)$ & & \\
\hline Níveis de TIBA|C1 & 2 & 1,87 & 0,93 & $0,91^{n s}$ \\
\hline Níveis de TIBA|C2 & 2 & 7,15 & 3,87 & $3,79^{*}$ \\
\hline ( Níveis de TIBA ) & (4) & 9,62 & & \\
\hline Resíduo & 266 & $271,02^{n s}$ & 1,02 & \\
\hline \multicolumn{3}{|c|}{$F_{(0,05 ; 1,266)}=3,88$} & $, 05 ; 2,266)=3,03$ & \\
\hline \multicolumn{2}{|c|}{$F_{(0,01 ; 1,266)}=6,73$} & & $, 01 ; 2,266)=4,68$ & \\
\hline
\end{tabular}

para o contraste testemunha vs [água de coco + TIBA] dentro do nível 1 do fator carvão, indicando que a testemunha difere dos demais tratamentos na ausência de carvão ativado. Observando-se a Tabela 12, que apresenta as médias dos tratamentos para a variável estudada, verifica-se que, dentro do nível 1 do fator carvão, o número médio de calos produzidos por explante é menor para a testemunha do que para os demais tratamentos. Por sua vez, para os demais tratamentos tem-se que o número médio de calos produzidos por explante é menor para TIBA do que para água de coco, os quais, conforme foi concluŕdo anteriormente, diferem entre si ao nível de $1 \%$ de probabilidade, independentemente do nível do fator carvão.

Quanto aos efeitos de níveis de água de coco e níveis de TIBA, tem- 
se que foram significativos ao nivel de $5 \%$ de probabilidade dentro do nível 2 do fator carvão. Fazendo-se um estudo de regressão para esses efeitos, os quais foram desdobrados em efeitos linear e quadrático, os resultados da análise de "deviance" correspondente apresentam-se na Tabela 14 e o programa em GLIM utilizado para efetuar esta análise apresenta-se no Apêndice 10.

Tabela 14: Resultados da análise de "deviance" para a variável número de calos produzidos por explante, do Conjunto B de dados, com desdobramento das interações em efeitos linear e quadrático, usando o modelo binomial negativo.

\begin{tabular}{|c|c|c|c|c|}
\hline Fontes de Variação & GL & "Deviance" & "Deviance" média & Razão \\
\hline Linear & 1 & 1,77 & 1,77 & $1,73^{\text {ns }}$ \\
\hline Quadrático & 1 & 2,12 & 2,12 & $2,08^{n s}$ \\
\hline ( Niveis de $\mathrm{AC} \mid \mathrm{C} 1$ ) & $(2)$ & $(3,89)$ & & \\
\hline Linear & 1 & 7,38 & 7,38 & $7,23^{* *}$ \\
\hline Quadrático & 1 & 0,06 & 0,06 & $0,06^{n s}$ \\
\hline ( Niveis de $\mathrm{AC} \mid \mathrm{C} 2$ ) & $(2)$ & $(7,44)$ & & \\
\hline Linear & 1 & 1,67 & 1,67 & $1,64^{n s}$ \\
\hline Quadrátido & 1 & 0,20 & 0,20 & $0,19^{n s}$ \\
\hline ( Níveis de TIBA|C1) & $(2)$ & $(1,87)$ & & \\
\hline Linear & 1 & 0,29 & 0,29 & $0,28^{n s}$ \\
\hline Quadrátido & 1 & 7,46 & 7,46 & $7,31^{* *}$ \\
\hline ( Niveis de TIBA|C2 ) & $(2)$ & $(7,75)$ & & \\
\hline Resíduo & 266 & $271,02^{n s}$ & 1,02 & \\
\hline
\end{tabular}

Considerando-se os níveis de água de coco e de TIBA dentro do nível 1 do fator carvão, tem-se que seus efeitos, linear e quadrático, não foram significativos ao nível de $5 \%$ de probabilidade. 
Para níveis de água de coco dentro do nivel 2 do fator carvão, o efeito quadrático não foi significativo ao nível de $5 \%$ de probabilidade, ao passo que o efeito linear foi significativo ao nível de $1 \%$ de probabilidade. A equação de regressão linear obtida é dada por:

$$
\hat{\eta}_{2 j}=\hat{\beta}_{0}+\hat{\beta}_{1} x_{j}=-0,42-0,11 x_{j}, \quad j=2,3,4,
$$

onde $\beta_{0}$ e $\beta_{1}$ são os coeficientes linear e angular, respectivamente, da equação de regressão linear e $x_{j}$ representa a concentração $j$ de água de coco no meio de cultura, em porcentagem, dentro do nível 2 do fator carvão, ou seja, $x_{2}=5 ; x_{3}=10 \mathrm{e}$ $x_{4}=15$.

As estimativas dos desvios-padrões para as estimativas dos parâmetros $\beta_{0}$ e $\beta_{1}$ da equação de regressão linear foram, respectivamente, 0,34 e 0,04 .

$O$ valor estimado para a observação $y_{2 j}, \hat{\mu}_{2 j}$, que representa o número médio de calos produzidos por explante de Stylosanthes scabra na presenço nível 2 do fator carvão, para a concentração $x_{j}$ de água de coco no meio de cultura, em porcentagem, $j=2,3,4$; é dado pela inversa da função de ligação do modelo, ou seja,

$$
\hat{\mu}_{2 j}=g^{-1}\left(\hat{\eta}_{2 j}\right)=k \frac{e^{\hat{\eta}_{2 j}}}{1-e^{\hat{\eta}_{2 j}}} .
$$

A Tabela 15 apresenta as médias dos tratamentos $T_{22}, T_{23}$ e $T_{24}$ para o número de calos produzidos por explante acompanhadas de seus valores estimados, dados pela expressão (28).

Tabela 15: Médias de tratamentos do Conjunto B de dados para a variável número de calos produzidos por explante, acompanhadas de seus valores estimados $\left(\hat{\mu}_{2 j}\right)$.

\begin{tabular}{ccc}
\hline Tratamento & Média & $\hat{\mu}_{2 j}$ \\
\hline 22 & 0,90 & 0,91 \\
23 & 0,45 & 0,42 \\
24 & 0,20 & 0,22 \\
\hline
\end{tabular}


O gráfico dos valores observados e dos valores estimados para o número médio de calos produzidos por explante de Stylosanthes scabra na presença de carvão ativado (nível 2 do fator carvão), em função da concentração $x$ de água de coco no meio de cultura, sendo $5<x<15$, em porcentagem, apresenta-se na Figura 21.

Voltando-se à Tabela 14, tem-se que, para níveis de TIBA dentro do nível 2 do fator carvão, o efeito quadrático foi significativo ao nível de $1 \%$ de probabilidade. Neste caso, não se prosseguiu com a análise, ou seja, não se obteve a equação quadrática de regressão, pois têm-se somente três pontos para efetuar o ajuste dessa equação. 


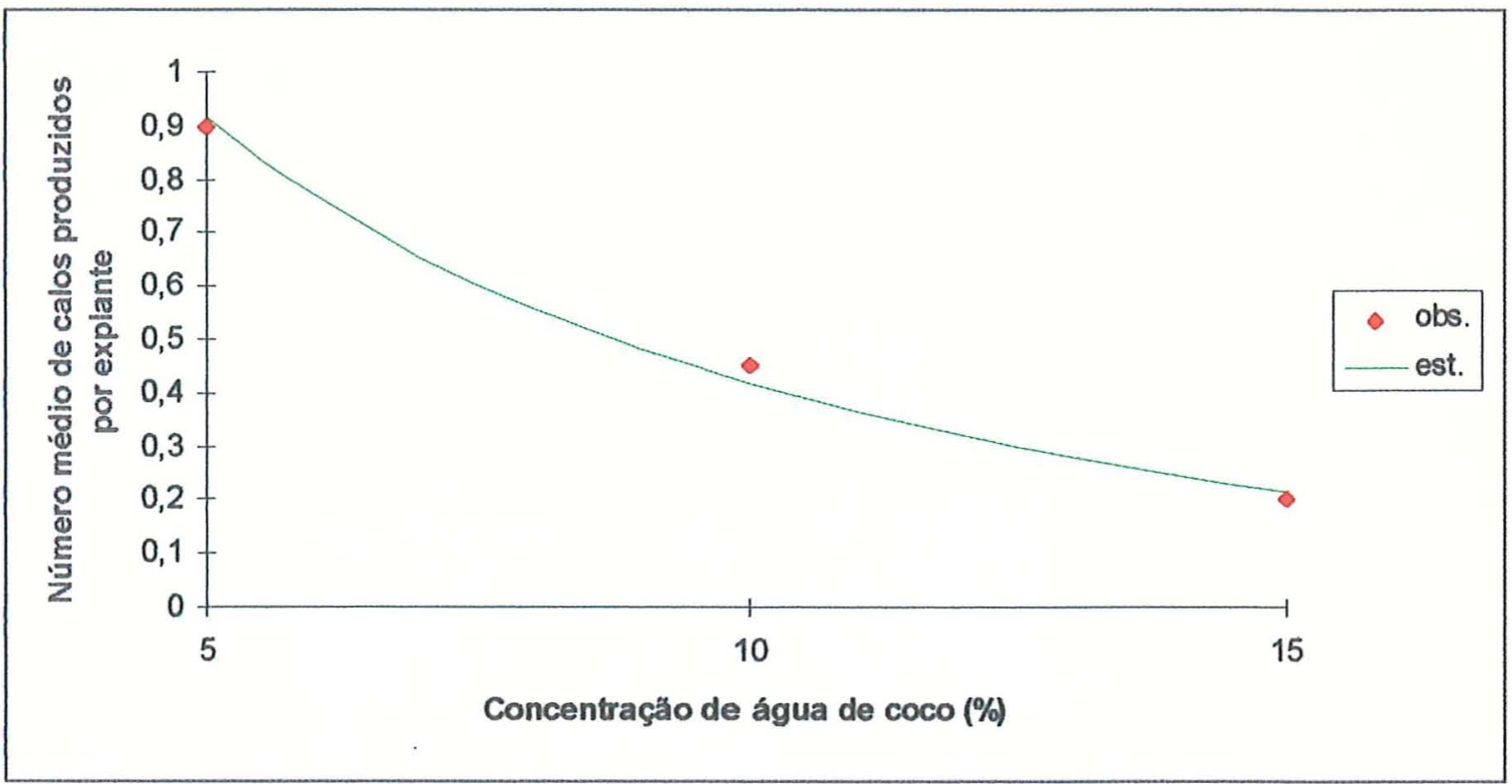

Figura 21: Gráfico dos valores observados e dos valores estimados para o número médio de calos produzidos por explante de Stylosanthes scabra na presença de carvão ativado, em função da concentração de água de coco no meio de cultura. 


\section{Conjunto C}

Dentre os 12 tratamentos deste conjunto de dados, 8 tiveram resultado significativo, ao nível de $5 \%$ de probabilidade, para o teste do cociente variânciamédia, conforme se verifica na Tabela 16

Dentre os 8 tratamentos que tiveram resultado significativo, em apenas um, o tratamento $T_{11}$, a hipótese favorecida com a rejeição de $H_{0}$ é a de que o tratamento tem média superior à variância. Ou seja, o resultado do teste indica a ocorrência de superdispersão neste conjunto de dados.

O teste para superdispersão, por sua vez, não foi significativo ao nível de $5 \%$ de probabilidade. $\mathrm{O}$ valor calculado para a estatística $T_{a}$ do teste e o valor crítico da distribuição normal padrão para $\alpha=0,05$ são:

$$
T_{a}=-0,85^{n s} \quad Z_{0,05}=1,64
$$

A discordância entre os resultados desses dois testes deve-se ao fato de que, no teste do cociente variância-média, ao serem consideradas todas as repetições dos tratamentos para o cálculo de suas médias e suas variâncias amostrais, tem-se que estas estatísticas contêm tanto o efeito de placa como o de explante dentro de placa, ao passo que o preditor linear considerado no ajuste do modelo Poisson para efetuar o teste para superdispersão, dado pela expressão (24), extrai da "deviance" residual do modelo o efeito de placa.

De fato, como mostram os resultados da análise de "deviance" efetuada para o modelo Poisson, os quais apresentam-se na Tabela 17, o teste de $\chi^{2}$ foi significativo ao nível de $1 \%$ de probabilidade para a "deviance" média de placa dentro de tratamento, associada a 32 graus de liberdade, e não foi significativo ao nível de $5 \%$ de probabilidade para a "deviance" residual, associada a 396 graus de liberdade, não rejeitando o modelo. No entanto, o teste $F$ para o efeito de placa dentro de tratamento foi significativo ao nível de $1 \%$ de probabilidade, de modo que os efeitos de espécie, de dose, e de interação entre espécie e dose devem ser testados com a "deviance" média de placa dentro de tratamento. 
Tabela 16: Teste do cociente variância-média aplicado aos tratamentos do Conjunto $\mathrm{C}$ de dados.

\begin{tabular}{|c|c|c|c|c|c|}
\hline Tratamento & $n$ & $\bar{y}$ & $s^{2}$ & $I(n-1)$ & $|d|$ \\
\hline 11 & 50 & 3,20 & 1,47 & & $3,14^{*}$ \\
\hline 21 & 40 & 0,62 & 1,83 & & $6,34^{*}$ \\
\hline 31 & 40 & 2,95 & 7,33 & & $5,15^{*}$ \\
\hline 41 & 40 & 0,17 & 0,35 & & $3,77^{*}$ \\
\hline 12 & 40 & 2,72 & 2,46 & & $0,38^{n s}$ \\
\hline 22 & 40 & 0,17 & 0,51 & & $6,26^{*}$ \\
\hline 32 & 40 & 3,57 & 15,07 & & $9,36^{*}$ \\
\hline 42 & 40 & 0,00 & 0,00 & & - \\
\hline 13 & 40 & 2,12 & 1,44 & & $1,49^{n s}$ \\
\hline 23 & 20 & 0,30 & 1,80 & $114,00^{*}$ & \\
\hline 33 & 10 & 5,50 & 5,61 & $9,18^{n s}$ & \\
\hline 43 & 40 & 1,17 & 2,15 & & $3,17^{*}$ \\
\hline \multicolumn{3}{|c|}{$\chi_{(0,975 ; 9)}^{2}=2,70$} & \multicolumn{2}{|c|}{$\chi_{(0,025 ; 9)}^{2}=19,04$} & \\
\hline \multicolumn{3}{|c|}{$\chi_{(0,975 ; 19)}^{2}=8,90$} & \multicolumn{2}{|c|}{$\chi_{(0,025 ; 19)}^{2}=32,87$} & \\
\hline & & $Z_{0}$ & 1,96 & & \\
\hline
\end{tabular}


Tabela 17: Resultados da análise de "deviance" para a variável número de calos produzidos por explante, do Conjunto $\mathrm{C}$ de dados, usando o modelo Poisson.

\begin{tabular}{lrccc}
\hline Fontes de Variação & GL & "Deviance" & "Deviance" média & Razão \\
\hline Espécie (E) & 3 & 506,30 & 168,88 & \\
Picloran (P) & 2 & 5,39 & 2,69 & \\
E x P & 6 & 105,82 & 17,64 & $12,66^{* *}$ \\
Placa|Trat & 32 & $336,30^{* *}$ & 10,51 & \\
Resíduo & 396 & $330,59^{\text {ns }}$ & 0,83 & \\
\hline Total & 439 & 1284,40 & \\
\hline \multicolumn{5}{c}{$\chi_{(0,05 ; 396)}^{2}=443,98$} \\
& $\chi_{(0,05 ; 32)}^{2}=46,26$ & $\chi_{(0,01 ; 32)}^{2}=53,53$ \\
$F_{(0,05 ; 32,396)}=1,47$ & $F_{(0,01 ; 32,396)}=1,72$
\end{tabular}

A partir desse resultado, adotou-se um novo modelo em que se tomou como variável resposta a média das 10 sub-amostras para cada placa ou repetição, e como preditor linear, $\eta$, o descrito a seguir:

$$
\eta_{i j k}=m+e_{i}+d_{j}+e d_{i j}
$$

onde $\eta_{i j k}$ é o preditor linear correspondente à observação $y_{i j k}=\bar{y}_{i j k .}, k=$ $1,2, \ldots, K_{i j}$, sendo os termos do preditor linear conforme descritos anteriormente no item 3.2 .

As médias do número de calos produzidos por explante para cada placa, ou repetição, apresentam-se na Tabela 23 , que consta do Apêndice 16.

O resultado obtido na análise de "deviance" para este novo modelo apresenta-se na Tabela 18. 
Tabela 18: Resultados da análise de "deviance" para as médias das sub-amostras das repetições do Conjunto $\mathrm{C}$ de dados, variável número de calos produzidos por explante, usando o modelo Poisson.

\begin{tabular}{lrccc}
\hline Fontes de Variação & GL & "Deviance" & "Deviance" média & Razão \\
\hline Espécie (E) & 3 & 50,63 & 16,88 & $16,08^{* *}$ \\
Picloran (P) & 2 & 0,54 & 0,27 & $0,26^{n s}$ \\
E x P & 6 & 10,58 & 1,76 & $1,68^{n s}$ \\
Resíduo & 32 & $33,63^{n s}$ & 1,05 & \\
\hline Total & 43 & 95,38 & & \\
\hline
\end{tabular}

$$
\begin{array}{lll} 
& \chi_{(0,05 ; 32)}^{2}=46,26 & \\
F_{(0,05 ; 2,32)}=3,29 & F_{(0,05 ; 3,32)}=2,90 & F_{(0,05 ; 6,32)}=2,40 \\
F_{(0,01 ; 2,32)}=5,34 & F_{(0,01 ; 3,32)}=4,46 & F_{(0,01 ; 6,32)}=3,43
\end{array}
$$

O teste de $\chi^{2}$ para a "deviance" residual do modelo, associada a 32 graus de liberdade, não foi significativo ao nível de $5 \%$ de probabilidade, indicando que o ajuste foi bom.

Os gráficos dos componentes de "deviance" padronizados versus índices e dos componentes de "deviance" estudentizados externamente versus índices que constam das Figuras 22 e 23, apresentam-se relativamente próximos de seu padrão ideal. Os pontos que aparecem distanciados dos demais, abaixo da reta de ordenada -2 , correspondem às observações de índices 28 e 29. Estas observações, cujos valores são 0,0 e 0,3 , respectivamente, ocorreram no tratamento $T_{32}$ junto aos dois maiores valores observados do conjunto de dados, ou seja, os valores 6,8 e 7,2. Daí aparecerem como pontos discrepantes. O gráfico normal de probabilidade para os valores absolutos dos componentes de "deviance" padronizados com envelope simulado, apresentado na Figura 25, mostra os pontos correspondentes às observações de índices 28 e 29 na parte superior direita do gráfico, indicando-as como "outliers". 


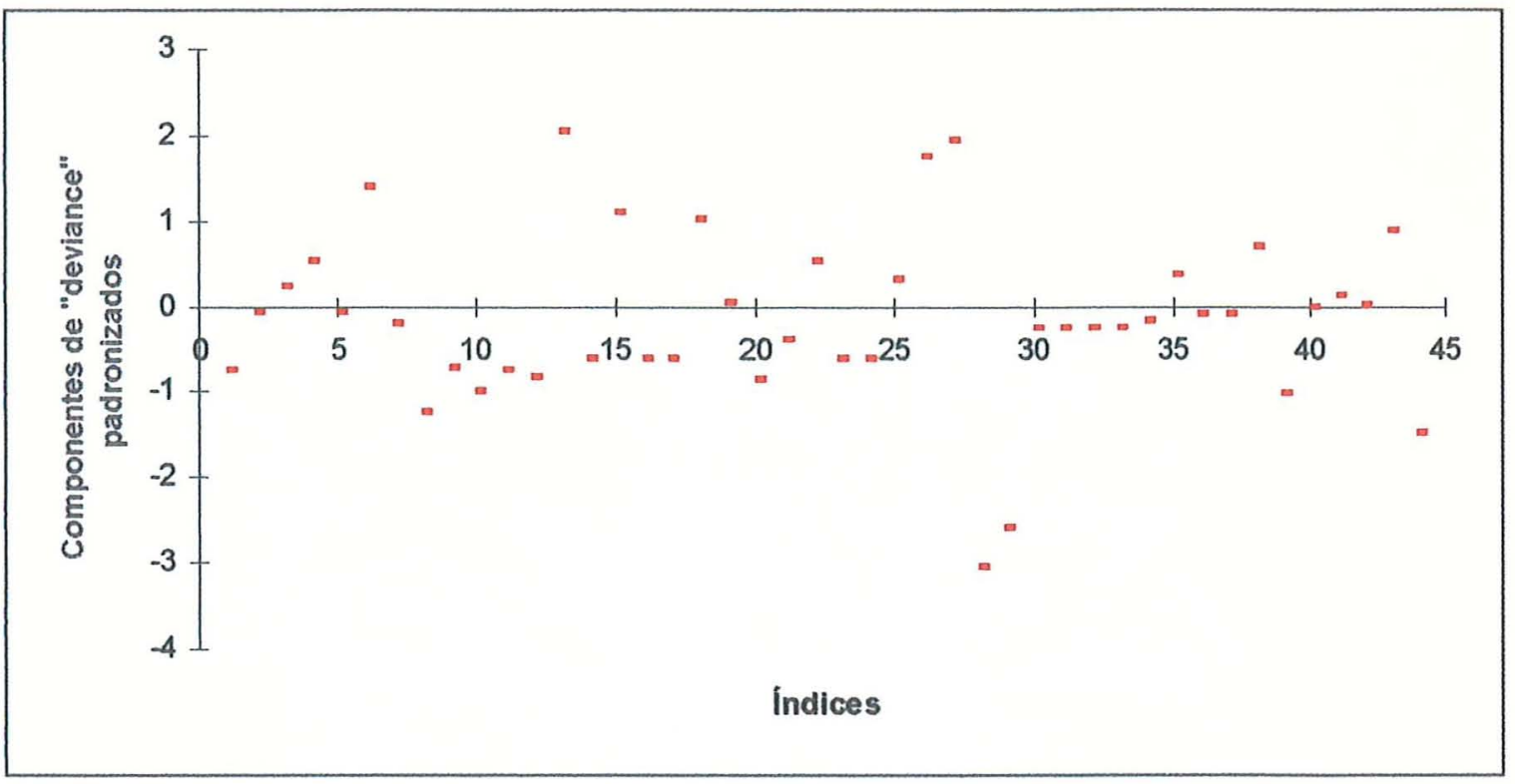

Figura 22: Gráfico dos componentes de "deviance" padronizados versus índices, resultante do ajuste do modelo Poisson ao Conjunto $\mathrm{C}$ de dados.

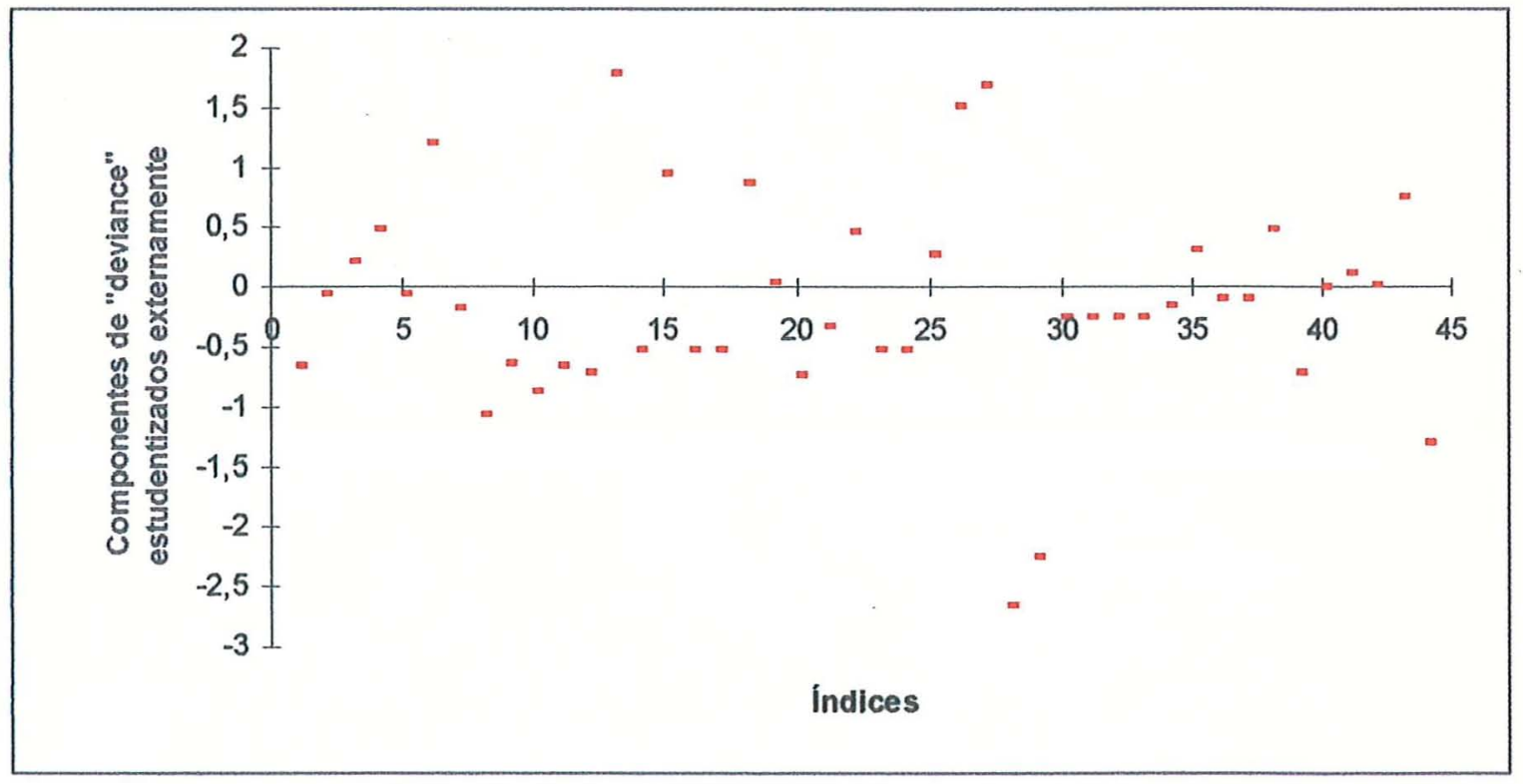

Figura 23: Gráfico dos componentes de "deviance" estudentizados externamente versus índices, resultante do ajuste do modelo Poisson ao Conjunto $\mathrm{C}$ de dados. 


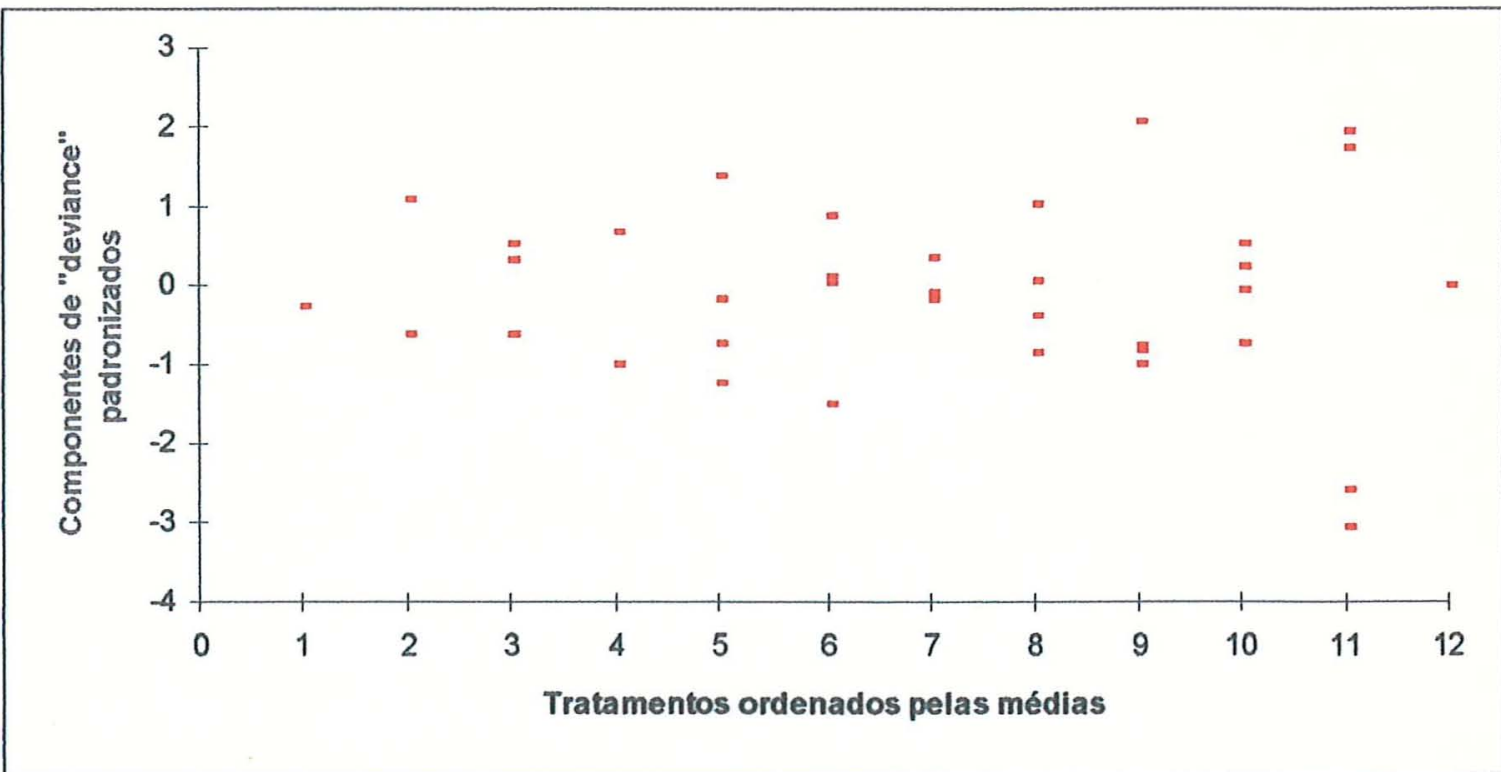

Figura 24: Gráfico dos componentes de "deviance" padronizados versus tratamentos ordenados pelas médias, resultante do ajuste do modelo Poisson ao Conjunto $\mathrm{C}$ de dados.

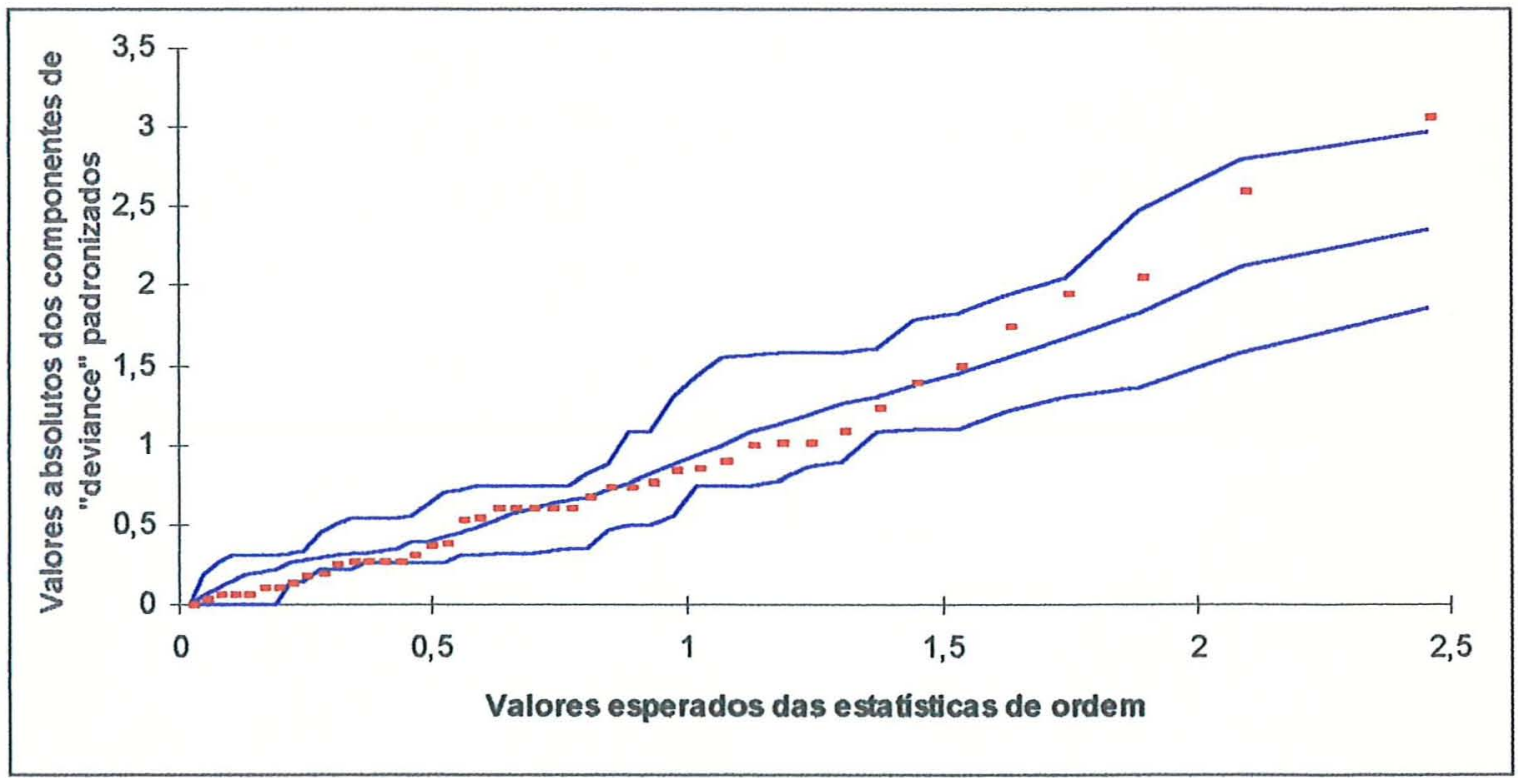

Figura 25: Gráfico normal de probabilidade para os valores absolutos dos componentes de "deviance" padronizados, resultante do ajuste do modelo Poisson ao Conjunto $\mathrm{C}$ de dados. 
No entanto, a não ser por estes pontos, este gráfico satisfaz seu padrão ideal, indicando que o ajuste do modelo foi bom.

O gráfico dos componentes de "deviance" padronizados versus tratamentos ordenados pelas médias, que consta da Figura 24, por sua vez, apresentase próximo de seu padrão ideal levando-se em consideração que a maior variação apresentada pelo tratamento que teve a segunda maior média, $T_{32}$, deve-se ao fato de as observações de índices 28 e 29 , indicadas como pontos discrepantes nos gráficos discutidos no parágrafo anterior, serem correspondentes a este tratamento. Os tratamentos ordenados pelas médias apresentam-se na Tabela 24, que consta do Apêndice 17. Quanto à coincidência dos pontos referentes ao tratamento que teve a menor média, $T_{42}$, esta deve-se ao fato de este tratamento apresentar todas suas observações iguais a zero.

O teste de $\chi^{2}$ para a "deviance" residual do modelo e a análise gráfica indicam que o ajuste deste modelo foi satisfatório. Em virtude disto, o modelo Poisson truncado não foi ajustado a este conjunto de dados.

$O$ teste $F$ aproximado aplicado às fontes de variação do modelo, conforme se verifica na Tabela 18, foi significativo somente para efeito de espécie, e, tendo em vista o objetivo do pesquisador, não há necessidade de se verificar como as espécies estudadas diferem. O experimento que deu origem a estes dados faz parte de uma pesquisa para o melhoramento genético da espécie Passiflora edulis através da embriogênese somática. Uma vez que a produção de calos é um processo que antecede a embriogênese somática, há interesse em se conhecer qual é a concentração do regulador de crescimento no meio de cultura que proporciona a maior produção de calos por explante para cada espécie. No entanto, não é de importância, neste caso, se uma espécie produz mais calos que outra, pois o interesse está em se transferir caracteristicas desejáveis, do ponto de vista industrial, das espécies $P$. quadrangularis, $P$. incarnata e $P$. maliformis para a espécie $P$. edulis, a única industrialmente interessante. 


\section{CONCLUSÕES}

De acordo com o que foi obtido neste trabalho, conclui-se que:

(a) Entre os modelos Poisson e Poisson truncado, aquele que melhor se ajustou ao Conjunto $\mathrm{A}$ de dados, que não apresenta superdispersão, foi o modelo Poisson truncado.

(b) Os modelos Poisson e Poisson truncado ajustaram-se pobremente ao Conjunto B de dados, que apresenta superdispersão.

(c) $\mathrm{O}$ modelo binomial negativo apresentou um ajuste razoalvelmente bom ao Conjunto $\mathrm{B}$ de dados.

(d) $\mathrm{O}$ modelo Poisson ajustou-se bem ao Conjunto $\mathrm{C}$ de dados, que não apresenta superdispersão.

(e) A utilização de modelos mais sofisticados em que o parâmetro $k$ da distribuição binomial negativa não é considerado fixo, mas sim estimado com os demais parâmetros desconhecidos do modelo, poderá ser uma alternativa melhor para o Conjunto B de dados, que apresentou superdispersão. 


\section{REFERÊNCIAS BIBLIOGRÁFICAS}

AITKIN, M; ANDERSON, D.; FRANCIS, B.; HINDE, J. Statistical modelling in GLIM. Oxford, Clarendon Press, 1989. 371p. (Oxford Statistical Science Ser.).

ATKINSON, A.C. Two graphical displays for outlying and influential observations in regression. Biometrika, 68(1): 13-20, 1981.

ATKINSON, A.C.; DAVISON, A.C.; NELDER, J.A.; O'BRIEN, C.M. Model checking (Skeleton notes). London, Imperial College of Science and Technology, 1989. 80p.

BRESLOW, N.E. Extra-Poisson variation in log-linear models. Applied Statistics, 33(1): 38-44, 1984.

CAMPBELL, M.J.; MACHIN, D.; D'ARCANGUES, C. Coping with extra Poisson variability in the analysis of factors influencing vaginal ring expulsions. Statistics in Medicine, 10: 241-54, 1991.

COLLET, D. Modelling binary data. London, Chapman \& Hall, 1991. 371p.

COLlingS, B.J. \& MARGOLIN, B.H. Testing goodness of fit for the Poisson assumption whem observations are not identically. Journal of the American Statistical Association, 80: 411-18, 1985.

DEAN, C. \& LAWLESS, J.F. Tests for detecting overdispersion in Poisson regres- 
sion models. Journal of the American Statistical Association, 84(406): 467-72, June. 1985.

DEMÉTRIO, C.G.B. Modelos lineares generalizados na experimentação agronômica. Porto Alegre, Departamento de Estatística da Universidade Federal do Rio Grande do Sul, 1993. 128p.

DIAZ, M. del P. Ajuste de modelos lineares generalizados para dados de contagem com superdispersão. Piracicaba, ESALQ/USP/Departamento de Matemática e Estatística, 1994.

ELLIOT, J.M. Some methods for the statistical analysis of samples of benthic invertebrates. 2.ed. Wareham, Freshwater Biological Association, 1979. 161p.

HINDE, J. Compound Poisson regression models. In: Gilchrist, R., ed. GLIM 82; Proceedings of the internacional conference on generalized linear models. New York, Springer-Verlog, 1982. p.109-121. (Lecture Notes in Statistics).

JOHNSON, N.L. \& KOTZ, S. Distributions in statistics: discrete distributions. New York, Houghton Mifflin, 1969. 328p.

LAMBERT, D. Zero-inflated Poisson regression, with an application to defects in manufacturing. Technometrics, 34: 1-14, 1992.

LARSON, H.J. Introduction to probability theory and statistical inference. 2.ed. New York, John Wiley, 1974. 431p. (Wiley Ser. in Probability and Mathematical Statistics).

McCULLAGH, P. \& NELDER, J.A. Generalized linear models. 2.ed. London, Chapman and Hall, 1989. 511p.

MOOD, A.M.; GRAYBILL, F.A.; BOES, D.C. Introduction to the theory of 
statistics. 3.ed. Singapore, McGraw-Hill, 1974. 564p.

MORGAN, B.J.T. Elements of simulation. London, Chapman and Hall, 1984. $351 \mathrm{p}$.

NELDER, J.A. \& WEDDERBURN, R.W.M. Generalized linear models. Journal of the Royal Statistical Society A, 135(3): 370-84, 1972.

PAYNE, C.D., ed. The GLIM system; release 3.77 manual. Oxford, Royal Statistical Society, 1986. 305p.

PIERCE, D.A. \& SHAFER, D.W. Residuals in generalized linear models. Journal of the American Statistical Association, 81: 977-86. 1986.

RIDOUT, M.S. \& DEMÉTRIO, C.G.B. Generalized linear models for positive count data. Revista de Matemática e Estatística, São Paulo, 10: 139-48, 1992.

RIDOUT, M.S. \& DEMÉTRIO, C.G.B. Letters to the editor. Statistics in Medicine, 13: 873-80, 1994.

SAS INSTITUTE INC. SAS procedures guide; release 6.03. Cary, 1988. 441p. 
APÊNDICES 
Apêndice

Página

1. Programa em SAS para cálculo da estatística do teste do cociente variância-média para a distribuição dos dados, aplicado ao Conjun-

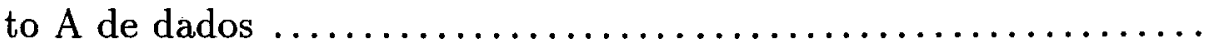

2. Programa em GLIM para cálculo da estatística $T_{a}$ do teste para superdispersão, aplicado ao Conjunto $\mathrm{A}$ de dados

3. Programa em GLIM para ajuste do modelo Poisson, aplicado ao Conjunto A de dados

4. Programa em GLIM para ajuste do modelo Poisson truncado, aplicado ao Conjunto A de dados

5. Programa em GLIM para o desdobramento dos efeitos principais dos fatores do modelo Poisson truncado ajustado ao Conjunto A de dados

6. Programa em GLIM para ajuste do modelo binomial ao Conjunto A de dados

7. Programa em GLIM para ajuste do modelo binomial negativo, aplicado ao Conjunto $\mathrm{B}$ de dados

8. Programa em GLIM para obtenção da "deviance" residual do modelo binomial negativo para 50 valores de $k$, aplicado ao Conjunto B de dados

9. Programa em GLIM para o desdobramento das interações do modelo binomial negativo ajustado ao Conjunto B de dados

10. Programa em GLIM para desdobramento das interações, em efeitos linear e quadrático, do modelo binomial negativo ajustado ao 
Conjunto B de dados

11. Macros para construção, no GLIM, do gráfico normal de probabilidade para os valores absolutos dos componentes de "deviance" padronizados com envelope simulado, para o modelo Poisson .....

12. Tabela 19 ...................................... 127

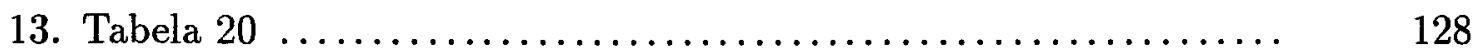

14. Tabela 21 ........................................ 129

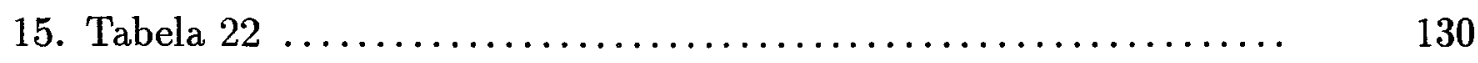

16. Tabela 23 ....................................... 133

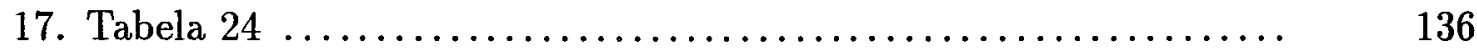




\begin{abstract}
APÊNDICE 1
Programa em SAS para cálculo da estatística do teste

do cociente variancia-média para a distribuição dos dados, aplicado ao

Conjunto A de dados
\end{abstract}

data primeiro;
input rad ana rep nc;
cards;
$\begin{array}{cccc}1 & 1 & 1 & 8 \\ 1 & 1 & 2 & 13 \\ 1 & 1 & 3 & 12 \\ 1 & 1 & 4 & 8 \\ 1 & 1 & 5 & 13 \\ 1 & 1 & 6 & 12 \\ 1 & 1 & 7 & 10 \\ 1 & 1 & 8 & 9 \\ 1 & 1 & 9 & 12 \\ 1 & 1 & 10 & 10 \\ 1 & 1 & 11 & 6 \\ 1 & 1 & 12 & 7 \\ 1 & 1 & 13 & 10 \\ 1 & 1 & 14 & 11 \\ 1 & 2 & 1 & 0 \\ 1 & 2 & 2 & 2 \\ 1 & 2 & 3 & 0 \\ 1 & 2 & 4 & 1 \\ 1 & 2 & 5 & 0 \\ 1 & 2 & 6 & 2\end{array}$




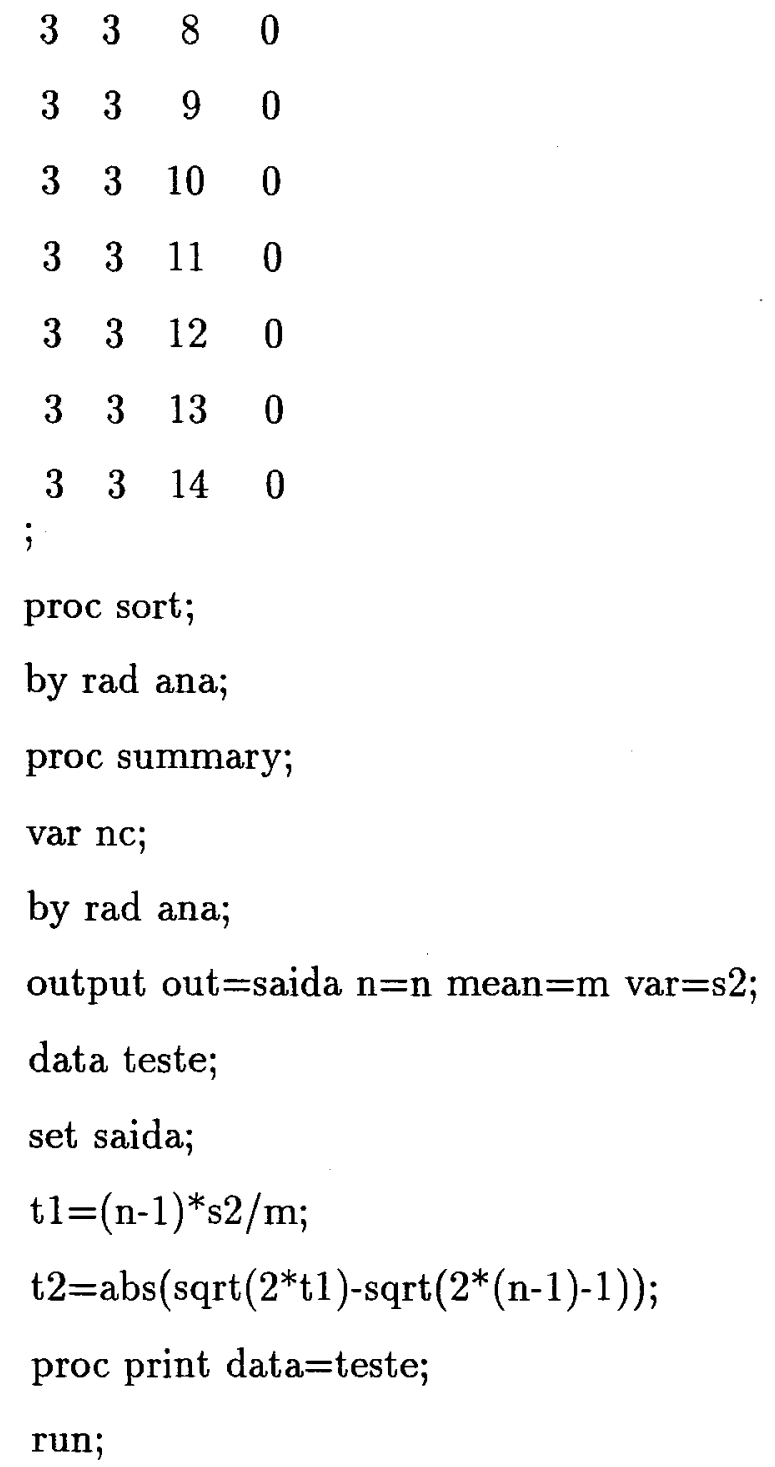




\section{APÊNDICE 2}

\section{Programa em GLIM para cálculo da estatística $T_{a}$}

do teste para superdispersão, aplicado ao Conjunto A de dados

\section{\$mac teste}

\$yvar nc

\$err $\mathrm{p}$

\$link 1

\$fit \# model

\$extrat \%vl

$\$ c a h=\%$ vl*\%wt $/ \%$ sc

$\$ c a \% a=\% c u\left((\% y v-\% \text { fv })^{* * 2-\% y v)}\right.$

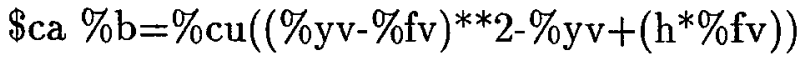

$\$ c a \% c=\% c u\left((\% \mathrm{fv})^{* * 2}\right)$

$\$$ ca $\% \mathrm{~s}=0.5^{*} \% \mathrm{a}$

$\$$ ca $\%$ t $=\%$ a $/ \%$ sqrt $(2 * \%$ c $)$

$\$$ ca $\% v=\%$ b/ $\% \operatorname{sqrt}(2 * \%$ c)

$\$$ print 'T='\%s

\$print 'T1='\%t

\$print 'Ta='\%v

\$endmac

!

\$mac model

\$fit : +rad : +ana : +rad.ana

$\$ \$$ endmac

!

\$units 127

\$data nc

\$read 


$\begin{array}{rrrrrrrrrrrrrrr}8 & 13 & 12 & 8 & 13 & 12 & 10 & 9 & 12 & 10 & 6 & 7 & 10 & 11 & \\ 0 & 2 & 0 & 1 & 0 & 2 & 0 & 1 & 0 & 1 & 0 & 2 & 3 & 0 & \\ 0 & 0 & 0 & 0 & 0 & 0 & 1 & 0 & 1 & 0 & 0 & 0 & 0 & 0 & \\ 6 & 8 & 7 & 8 & 10 & 11 & 10 & 12 & 10 & 11 & 6 & 13 & 6 & 8 & 9 \\ 0 & 2 & 3 & 0 & 0 & 0 & 0 & 0 & 0 & 0 & 0 & 0 & 0 & 0 & \\ 2 & 0 & 0 & 0 & 0 & 0 & 0 & 0 & 0 & 0 & 0 & 0 & 0 & 0 & \\ 0 & 0 & 0 & 1 & 0 & 0 & 1 & 0 & 2 & 0 & 0 & 0 & 0 & 0 & \\ 0 & 0 & 0 & 0 & 0 & 0 & 0 & 0 & 0 & 0 & 0 & 0 & 0 & 0 & \\ 0 & 0 & 0 & 0 & 0 & 0 & 0 & 0 & 0 & 0 & 0 & 0 & 0 & 0\end{array}$

$\$ \operatorname{var} 42$ a c : $43 \mathrm{~b}: 14$ e f g : $15 \mathrm{~h}$

$\$$ ca $a=1: b=2: c=3: e=1: f=2: g=3: h=1$

$\$$ ca $i=e^{*} 11: j=e^{*} 12: k=e^{*} 13: l=h^{*} 21: m=e^{*} 22: n=e^{*} 23:$

$$
\mathrm{o}=\mathrm{e}^{*} 31: \mathrm{p}=\mathrm{e}^{*} 32: \mathrm{q}=\mathrm{e}^{*} 33
$$

\$assign $\operatorname{rad}=a, b, c:$ ana $=e, f, g, h, f, g, e, f, g:$ trat=i,j,k,l,m,n,o,p,q

$\$$ del h

\$factor rad 3: ana 3

\$use teste

\$finish 


\section{APÊNDICE 3}

\section{Programa em GLIM para ajuste do modelo Poisson, aplicado ao Conjunto $\mathrm{A}$ de dados}

\$mac poisson

\$yvar nc

\$err p

$\$$ link 1

\$fit \# model

\$extrat \%vl

$\$ \mathrm{ca} \mathrm{hi}=\% \mathrm{vl}^{*} \%$ wt $/ \% \mathrm{sc}$

$\$$ ca ro $=\% y v-\%$ fv

$\$$ ca $r p=$ ro $/ \%$ sqrt $(\%$ fv $)$

$\$$ ca $\operatorname{rpp}=\operatorname{rp} / \% \operatorname{sqrt}((1-h i) * \%$ sc $)$

$\$$ ca $y v=\%$ if $(\%$ eq $(\% y v, 0), 0.001, \% y v)$

$\$$ ca $\mathrm{cd}=\% \operatorname{sqrt}\left(2^{*}((\% \mathrm{yv} * \% \log (\mathrm{yv} / \% \mathrm{fv}))-\% \mathrm{yv}+\% \mathrm{fv})\right): \mathrm{cd}=\% \operatorname{if}(\% \mathrm{lt}(\mathrm{ro}, 0),-\mathrm{cd}, \mathrm{cd})$

$\$$ ca $\mathrm{cdp}=\mathrm{cd} / \% \operatorname{sqrt}((1-\mathrm{hi}) * \% \mathrm{sc})$

$\$ c a$ lik $=\%$ sqrt $\left(\left(h^{*}{ }^{*}\right.\right.$ rpp $^{*}$ rpp $\left.)+(1-h i)^{*} \operatorname{cdp}{ }^{*} \operatorname{cdp}\right): \operatorname{lik}=\%$ if $(\% \operatorname{lt}($ ro, 0$),-l i k, l i k)$

$\$$ look trat nc \%fv \%lp cdp lik

$\$ \$$ endmac

!

\$mac model

\$fit : +rad : +ana : +rad.ana

$\$ \$$ endmac

!

\$units 127

$\$$ data nc

\$read 


$\begin{array}{rrrrrrrrrrrrrrr}8 & 13 & 12 & 8 & 13 & 12 & 10 & 9 & 12 & 10 & 6 & 7 & 10 & 11 & \\ 0 & 2 & 0 & 1 & 0 & 2 & 0 & 1 & 0 & 1 & 0 & 2 & 3 & 0 & \\ 0 & 0 & 0 & 0 & 0 & 0 & 1 & 0 & 1 & 0 & 0 & 0 & 0 & 0 & \\ 6 & 8 & 7 & 8 & 10 & 11 & 10 & 12 & 10 & 11 & 6 & 13 & 6 & 8 & 9 \\ 0 & 2 & 3 & 0 & 0 & 0 & 0 & 0 & 0 & 0 & 0 & 0 & 0 & 0 & \\ 2 & 0 & 0 & 0 & 0 & 0 & 0 & 0 & 0 & 0 & 0 & 0 & 0 & 0 & \\ 0 & 0 & 0 & 1 & 0 & 0 & 1 & 0 & 2 & 0 & 0 & 0 & 0 & 0 & \\ 0 & 0 & 0 & 0 & 0 & 0 & 0 & 0 & 0 & 0 & 0 & 0 & 0 & 0 & \\ 0 & 0 & 0 & 0 & 0 & 0 & 0 & 0 & 0 & 0 & 0 & 0 & 0 & 0 & \end{array}$

$\$ \operatorname{var} 42$ a c : 43 b : 14 e f g : $15 \mathrm{~h}$

$\$ c a \quad a=1: b=2: c=3: e=1: f=2: g=3: h=1$

\$ca $\mathrm{i}=\mathrm{e}^{*} 11: \mathrm{j}=\mathrm{e}^{*} 12: \mathrm{k}=\mathrm{e}^{*} 13: \mathrm{l}=\mathrm{h}^{*} 21: \mathrm{m}=\mathrm{e}^{*} 22: \mathrm{n}=\mathrm{e}^{*} 23$ :

$$
\mathrm{o}=\mathrm{e}^{*} 31: \mathrm{p}=\mathrm{e}^{*} 32: \mathrm{q}=\mathrm{e}^{*} 33
$$

\$assign $\operatorname{rad}=a, b, c:$ ana $=e, f, g, h, f, g, e, f, g:$ trat $=i, j, k, l, m, n, o, p, q$

$\$$ factor rad 3 : ana 3

\$use poisson

\$finish 


\section{APÊNDICE 4 \\ Programa em GLIM para ajuste do modelo Poisson truncado, aplicado ao Conjunto A de dados}

$\$$ mac pt

$\$ y v a r n c$

\$own fv dr va di

$\$$ ca $\%$ yv $=\%$ if $(\%$ eq $(\%$ yv, 1$), 1.001, \%$ yv $)$

$\$ c a \% \operatorname{lp}=\% \log (\% \mathrm{yv}-1)$

\$sca 1

\$fit \# model

Sextrat \%vl

$\$$ ca hi $=\%$ vl $* \%$ wt $/ \%$ sc

$\$$ ca ro $=\%$ yv- $\%$ fv

$\$$ ca rp $=$ ro $/ \%$ sqrt $\left(\% \mathrm{fv}^{*}(1+\right.$ lhat $-\%$ fv $\left.)\right)$

$\$$ ca $r p p=r p / \% \operatorname{sqrt}((1-h i) * \%$ sc $)$

$\$$ ca cd $=2 *((\%$ yv $* \% \log (\operatorname{lstar} / \operatorname{lhat}))-\% \log ((\% \exp (\operatorname{lstar})-1) /(\% \exp ($ lhat $)-1)))$ :

$$
\mathrm{cd}=\% \operatorname{sqrt}(\mathrm{cd}): \mathrm{cd}=\% \operatorname{if}(\% \mathrm{lt}(\mathrm{ro}, 0),-\mathrm{cd}, \mathrm{cd})
$$

$\$$ ca $\operatorname{cdp}=\mathrm{cd} / \% \operatorname{sqrt}((1-\mathrm{hi}) * \% \mathrm{sc})$

\$ca lik $=\%$ sqrt $\left(\left(\right.\right.$ hi $\left.\left.^{*}{ }^{\mathrm{rpp}}{ }^{*} \mathrm{rpp}\right)+(1-\mathrm{hi})^{*} \mathrm{cdp}{ }^{*} \mathrm{cdp}\right)$ :

$$
\text { lik }=\% \text { if(\%lt }(\text { ro, } 0),- \text { lik,lik })
$$

\$look trat nc \%fv \%lp cdp lik

\$endmac

!

\$mac lambda

$\$$ ca o=\% $1 *(1-\% \exp (1 / \% 1-\% 1))$

$\$$ ca $\% 2=\% 1^{*}(1-\% \exp (-\mathrm{o}))^{* *} 2-$ o $^{*}{ }^{*} \% \exp (-\mathrm{o})$

$\$$ ca $\% 2=\% 2 /(1-(o+1) * \% \exp (-o)+\% \operatorname{eq}(\% 1,1))$

$\$ \$ e n d m a c$ 
$\$ \mathrm{mac} f \mathrm{v}$

$\$ c a \% f v=\% \exp (\% \mathrm{lp})+1 \$ \$ \mathrm{e}$

!

$\$ \mathrm{mac} d \mathrm{r}$

$\$ c a \% d r=1 /(\%$ fv- 1$) \$ \$$ e

!

\$mac va

\$use lambda \%fv lhat

$\$ \mathrm{ca} \% \mathrm{va}=\% \mathrm{fv} *(1+\mathrm{lhat}-\% \mathrm{fv}) \$ \$ \mathrm{e}$

!

$\$$ mac di

\$use lambda \%yv lstar

$\$$ ca $\% \mathrm{di}=2 * \% \mathrm{yv} * \% \log ($ lstar $/$ lhat $)$ :

$\% \mathrm{di}=(\% \mathrm{di}-2 * \% \log ((\% \exp ($ lstar $)-1) /(\% \exp ($ lhat $)-1))) \$ \$ \mathrm{e}$

!

\$mac model

\$fit : +rad : +ana : +rad.ana

$\$ \$ e n d m a c$

!

\$units 44

\$data nc

\$read 


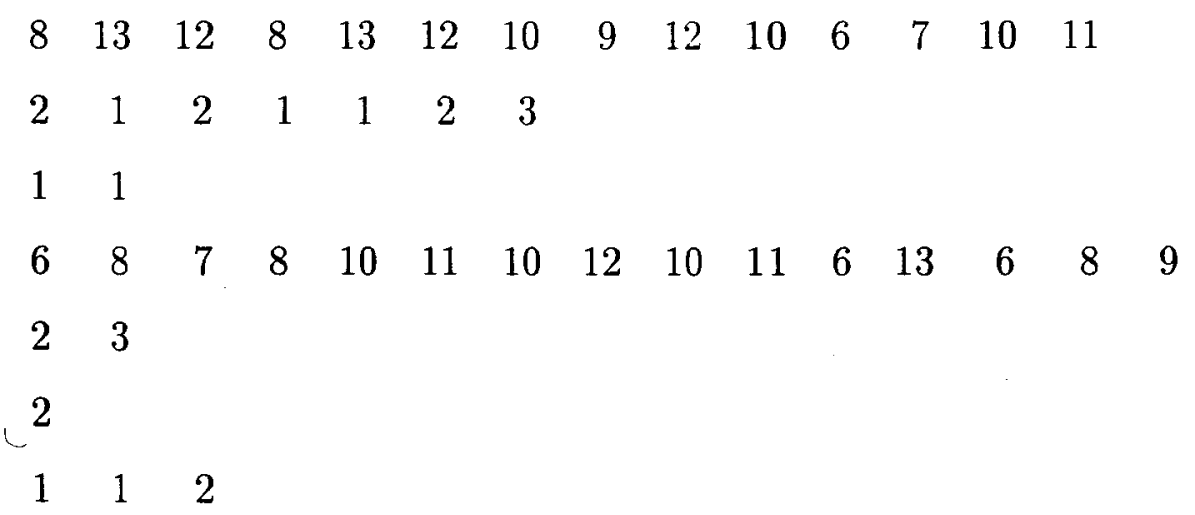

$\$ \operatorname{var} 23 \mathrm{a}: 18 \mathrm{~b}: 3 \mathrm{c}: 14 \mathrm{e}: 7 \mathrm{f}: 2 \mathrm{~g}: 15 \mathrm{~h}: 2 \mathrm{j}: 1 \mathrm{k} \mathrm{s}: 31$

$\$ c a \quad a=1: b=2: c=3: e=1: f=2: g=3: h=1: j=2: k=3: 1=1:$

$$
\begin{aligned}
& \mathrm{m}=\mathrm{e}^{*} 11: \mathrm{n}=\mathrm{f}^{*} 6: \mathrm{p}=\mathrm{g}^{*} 13 / 3: \mathrm{q}=\mathrm{h}^{*} 21: \mathrm{r}=\mathrm{j}^{*} 11: \\
& \mathrm{s}=23: \mathrm{t}=\mathrm{l}^{*} 31
\end{aligned}
$$

\$assign $\mathrm{rad}=\mathrm{a}, \mathrm{b}, \mathrm{c}:$ ana $=\mathrm{e}, \mathrm{f}, \mathrm{g}, \mathrm{h}, \mathrm{j}, \mathrm{k}, \mathrm{l}:$ trat $=\mathrm{m}, \mathrm{n}, \mathrm{p}, \mathrm{q}, \mathrm{r}, \mathrm{s}, \mathrm{t}$

$\$$ factor rad $3:$ ana 3

Suse pt

\$finish 


\begin{abstract}
APÊNDICE 5
Programa em GLIM para o desdobramento

dos efeitos principais dos fatores do modelo Poisson truncado

ajustado ao Conjunto $\mathbf{A}$ de dados
\end{abstract}

\$mac lambda

\$ca $0=\% 1 *(1-\% \exp (1 / \% 1-\% 1))$

\$ca $\% 2=\% 1^{*}(1-\% \exp (-\mathrm{o}))^{* *} 2-\mathrm{o}^{*} \mathrm{o} * \% \exp (-\mathrm{o})$

$\$$ ca $\% 2=\% 2 /(1-(\mathrm{o}+1) * \% \exp (-\mathrm{o})+\%$ eq $(\% 1,1))$

$\$ \$$ endmac

!

$\$ \mathrm{mac} f \mathrm{v}$

$\$ c a \% f v=\% \exp (\% l p)+1 \$ \$ \mathrm{e}$

!

$\$ \operatorname{mac} d r$

$\$ c a \% d r=1 /(\% \mathrm{fv}-1) \$ \$ \mathrm{e}$

$!$

\$mac va

Suse lambda \%fv lhat

$\$$ ca $\%$ va $=\% \mathrm{fv}^{*}(1+$ lhat $-\% \mathrm{fv}) \$ \$ \mathrm{e}$

!

$\$$ mac di

\$use lambda \%yv lstar

Sca $\%$ di $=2 * \% y v * \% \log ($ lstar $/$ lhat $)$ :

$\% \mathrm{di}=(\% \mathrm{di}-2 * \% \log ((\% \exp (1 \mathrm{star})-1) /(\% \exp ($ lhat $)-1))) \$ \$ \mathrm{e}$

$!$

Sunits 44

\$data nc

\$read 


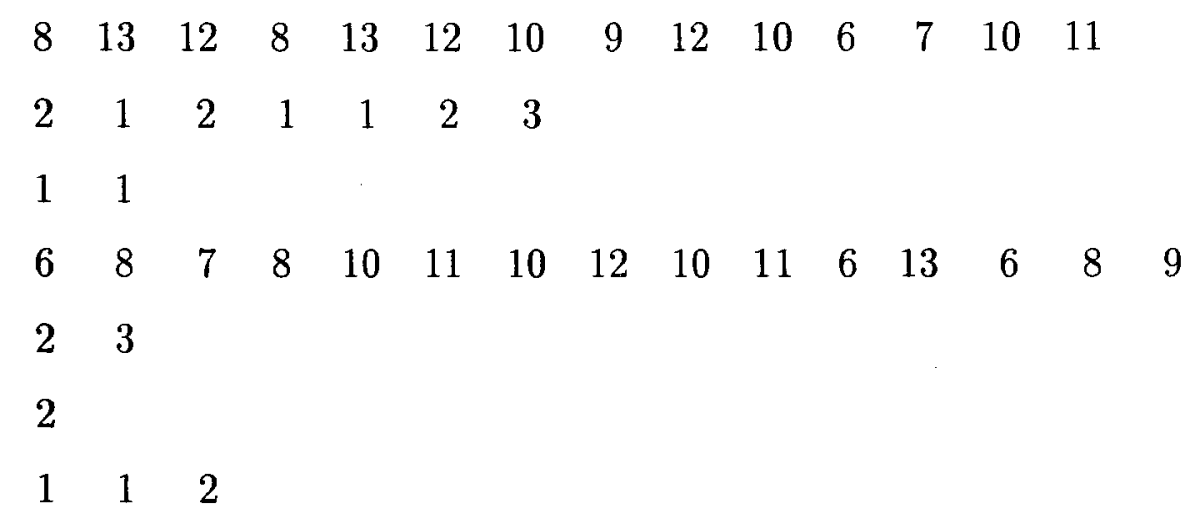

$\$ \operatorname{var} 23 \mathrm{a}: 18 \mathrm{~b}: 3 \mathrm{c}: 14 \mathrm{e}: 7 \mathrm{f}: 2 \mathrm{~g}: 15 \mathrm{~h}: 2 \mathrm{j}: 1 \mathrm{k} \mathrm{s}: 31$

$\$ c a \quad a=1: b=2: c=3: e=1: f=2: g=3: h=1: j=2: k=3: l=1:$

$\mathrm{m}=\mathrm{e}^{*} 11: \mathrm{n}=\mathrm{f}^{*} 6: \mathrm{p}=\mathrm{g}^{*} 13 / 3: \mathrm{q}=\mathrm{h}^{*} 21: \mathrm{r}=\mathrm{j}^{*} 11: \mathrm{s}=23: \mathrm{t}=\mathrm{l}^{*} 31$

\$assign $\mathrm{rad}=\mathrm{a}, \mathrm{b}, \mathrm{c}:$ ana $=\mathrm{e}, \mathrm{f}, \mathrm{g}, \mathrm{h}, \mathrm{j}, \mathrm{k}, \mathrm{l}:$ trat $=\mathrm{m}, \mathrm{n}, \mathrm{p}, \mathrm{q}, \mathrm{r}, \mathrm{s}, \mathrm{t}$

\$factor rad 3 : ana 3

\$yvar nc

\$own fv dr va di

\$ca $\%$ yv $=\%$ if $(\%$ eq(\%yv, 1$), 1.001, \%$ yv $)$

$\$ c a \% \operatorname{lp}=\% \log (\% \mathrm{yv}-1)$

\$sca 1

\$fit : +rad : +ana : +rad.ana

!

\$print '(R1+R2) VS R3'

$\$ c a b=b / 2: c=(c / 3)^{*} 2$

\$assign c1=a,b,c

\$factor c1 2

\$fit : $+\mathrm{c} 1$

!

\$print 'R1 VS R2'

$\$ c a$ w $=\%$ if $(\%$ eq $(c 1,2), 0,1): b=b^{*} 2$

\$assign c2=a,b,c

$\$$ factor c2 2 
\$wei w

\$fit : $+\mathrm{c} 2$

$!$

\$print 'REGRESSAO PARA NIVEIS DE ANA'

$\$$ ca $e=0: f=0.5: g=1: h=0: j=0.5: k=1: l=0$

\$assign al=e,f,g,h,j,k,l

$\$ c a$ aq $=$ al $^{*}$ al

\$wei

\$fit : $\operatorname{trad}:+$ al $:+$ aq

$\$$ ca $e=1: f=1: g=1: h=1: j=1: k=1: 1=0$

\$assign $w=e, f, g, h, j, k, l$

\$wei w

\$fit : +al

$\$$ display ev

\$look \%lp \%fv

\$finish 


\title{
APÊNDICE 6
}

\section{Programa em GLIM para ajuste do modelo binomial ao Conjunto A de dados}

\author{
Sunits 9 \\ \$data rad ana $\mathbf{r} \mathbf{n}$ \\ \$read \\ $\begin{array}{llll}1 & 1 & 14 & 14\end{array}$ \\ $\begin{array}{llll}1 & 2 & 7 & 14\end{array}$ \\ $\begin{array}{llll}1 & 3 & 2 & 14\end{array}$ \\ $\begin{array}{llll}2 & 1 & 15 & 15\end{array}$ \\ $\begin{array}{llll}2 & 2 & 2 & 14\end{array}$ \\ $\begin{array}{llll}2 & 3 & 1 & 14\end{array}$ \\ $\begin{array}{llll}3 & 1 & 3 & 14\end{array}$ \\ $\begin{array}{llll}3 & 2 & 0 & 14\end{array}$ \\ $\begin{array}{llll}3 & 3 & 0 & 14\end{array}$ \\ $\$$ ca $r=\%$ if $(\%$ eq $(r, 0), 0.01, r)$ \\ $\$$ factor rad 3 : ana 3 \\ \$yvar r \\ Serror b n \\ \$link g \\ \$fit : $\operatorname{trad}: \operatorname{tana}$ \\ ! \\ \$print '(R1+R2) VS R3' \\ $\$$ assign $\mathrm{cl}=1,1,1,1,1,1,2,2,2$ \\ \$factor c1 2 \\ \$fit : $+\mathrm{c1}$ \\ ! \\ \$print 'R1 VS R2'
}


\$ca $\mathrm{w}=\%$ if $(\%$ eq $(\mathrm{c} 1,2), 0,1)$

\$assign c2=1,1,1,2,2,2,2,2,2

\$factor c2 2 \$wei w

\$fit : + c2

!

\$print 'REGRESSAO PARA NIVEIS DE ANA'

\$assign al $=0,0.5,1,0,0.5,1,0,0.5,1$

$\$ c a$ aq $=a l^{*}$ al

\$wei

\$fit $:+\operatorname{rad}:+$ al $:+$ aq

\$finish 


\section{APÊNDICE 7}

\section{Programa em GLIM para ajuste do modelo binomial negativo, aplicado ao Conjunto $B$ de dados}

\$mac bn

\$yvar nc

\$own fv dr va di

$\$ c a$ yv $=\%$ if $(\%$ eq $(\% y v, 0), 0.001, \%$ yv $)$

$\$$ ca $\% \mathrm{lp}=\% \log (\mathrm{yv} /(\mathrm{yv}+\% \mathrm{k}))$

\$sca 1

\$it \# model

Sextrat \%vl

$\$ c a$ hi $=\%$ vl*\%wt $/ \%$ sc

\$ca ro $=\% \mathrm{yv}-\% \mathrm{fv}$

$\$ c a$ rp=ro/\%sqrt $\left(\% f_{v}^{*}(1+(\% \mathrm{fv} / \% \mathrm{k}))\right)$

$\$$ ca $\mathrm{rpp}=\mathrm{rp} / \%$ sqrt $((1-\mathrm{hi}) * \%$ sc $)$

$\$$ ca $\mathrm{cd}=2^{*}(\% \mathrm{yv} * \% \log (\mathrm{yv} / \% \mathrm{fv})-(\% \mathrm{yv}+\% \mathrm{k}) * \% \log ((\% \mathrm{yv}+\% \mathrm{k}) /(\% \mathrm{fv}+\% \mathrm{k})))$ :

$$
\text { cd }=\% \text { sqrt(cd) : cd=\%if(\%lt(ro,0),-cd,cd) }
$$

$\$$ ca cdp $=$ cd $/ \%$ sqrt $((1-$ hi $) * \%$ sc $)$

$\$$ ca lik=\%sqrt ((hi* ${ }^{*}{ }^{*}{ }^{*}$ rpp $)+(1-h i)^{*} \operatorname{cdp}^{*}$ cdp $)$ :

$$
\text { lik }=\% \text { if }(\% \operatorname{lt}(\text { ro, }, 0),- \text { lik,lik })
$$

\$look trat nc \%fv \%lp cdp lik

$\$ \$$ endmac

!

$\$ \operatorname{mac} f v$

$\$$ ca $\% \mathrm{fv}=\% \mathrm{k}^{*} \% \exp (\% \mathrm{lp}) /(1-\% \exp (\% \mathrm{lp})) \$ \$ \mathrm{e}$

!

$\$ \operatorname{mac} d r$

$\$ \mathrm{ca} \% \mathrm{dr}=\% \mathrm{k} /\left(\% \mathrm{fv}^{*}(\% \mathrm{fv}+\% \mathrm{k})\right) \$ \$ \mathrm{e}$ 


\section{!}

\$mac va

$\$ c a \%$ va $=\%$ fv $*(1+(\%$ fv $/ \% \mathrm{k})) \$ \$$ e

!

$\$$ mac di

$\$ c a \%$ di $=2^{*}(\% \mathrm{yv} *(\% \log (\mathrm{yv} / \% \mathrm{fv}))-(\% \mathrm{yv}+\% \mathrm{k}) * \% \log ((\% \mathrm{yv}+\% \mathrm{k}) /(\% \mathrm{fv}+\% \mathrm{k}))) \$ \$ \mathrm{e}$

!

\$mac model

\$wei

\$fit : +carvao : +supl : +carvao.supl

$\$ \$$ endmac

!

\$units 280

\$data nc

Sread

$\begin{array}{rrrrrrrrrrrrrrrrrrrr}10 & 6 & 10 & 2 & 5 & 5 & 3 & 4 & 8 & 2 & 2 & 2 & 2 & 4 & 2 & 1 & 11 & 2 & 3 & 2 \\ 50 & 30 & 35 & 20 & 15 & 16 & 35 & 25 & 10 & 10 & 15 & 10 & 25 & 30 & 30 & 20 & 30 & 20 & 20 & 32 \\ 80 & 80 & 30 & 45 & 10 & 10 & 80 & 10 & 60 & 65 & 45 & 29 & 15 & 10 & 10 & 16 & 70 & 10 & 40 & 80 \\ 70 & 72 & 10 & 10 & 60 & 40 & 39 & 20 & 50 & 25 & 14 & 10 & 45 & 10 & 50 & 10 & 60 & 20 & 35 & 60 \\ 22 & 12 & 30 & 40 & 2 & 2 & 1 & 13 & 18 & 3 & 20 & 2 & 14 & 35 & 7 & 1 & 1 & 1 & 1 & 10 \\ 18 & 15 & 15 & 30 & 7 & 3 & 12 & 20 & 18 & 18 & 2 & 10 & 6 & 25 & 1 & 4 & 2 & 45 & 40 & 2 \\ 20 & 12 & 15 & 3 & 32 & 1 & 19 & 8 & 1 & 1 & 1 & 23 & 50 & 20 & 30 & 15 & 20 & 7 & 13 & 50 \\ 4 & 0 & 1 & 0 & 0 & 2 & 2 & 0 & 0 & 2 & 0 & 0 & 0 & 1 & 0 & 0 & 4 & 1 & 0 & 1 \\ 0 & 0 & 1 & 0 & 1 & 0 & 0 & 4 & 0 & 3 & 0 & 0 & 3 & 0 & 0 & 0 & 0 & 2 & 2 & 2 \\ 1 & 0 & 0 & 0 & 0 & 0 & 3 & 0 & 1 & 0 & 0 & 0 & 0 & 0 & 0 & 0 & 0 & 0 & 1 & 3 \\ 0 & 0 & 0 & 0 & 1 & 0 & 0 & 0 & 0 & 0 & 0 & 0 & 0 & 0 & 0 & 0 & 0 & 1 & 0 & 2 \\ 0 & 1 & 0 & 0 & 0 & 0 & 1 & 0 & 0 & 0 & 0 & 0 & 0 & 0 & 0 & 0 & 0 & 0 & 1 & 0 \\ 0 & 0 & 1 & 2 & 1 & 1 & 0 & 0 & 0 & 0 & 0 & 0 & 0 & 0 & 0 & 0 & 0 & 0 & 5 & 6 \\ 0 & 0 & 0 & 1 & 2 & 2 & 0 & 0 & 0 & 0 & 0 & 0 & 0 & 0 & 0 & 0 & 1 & 0 & 2 & 0\end{array}$

$\$ \operatorname{var} 140$ a b c

$\$ c a \quad a=\% g l(7,20): b=a+10: c=a+20$

\$assign trat=b,c 
$\$$ del a b c

$\$$ ca $\operatorname{carvao}=\% \operatorname{gl}(2,140): \operatorname{supl}=\% \operatorname{gl}(7,20)$

\$factor carvao 2 : supl 7

$\$$ ca $\% \mathrm{k}=$ ! atribuir a $\% \mathrm{k}$ o valor do parametro $\mathrm{k}$ !

\$yvar nc

\$own fv dr va di

$\$$ ca $y v=\%$ if $(\%$ eq $(\% y v, 0), 0.001, \% y v)$

$\$$ ca $\% \operatorname{lp}=\% \log (\mathrm{yv} /(\mathrm{yv}+\% \mathrm{k}))$

\$sca 1

\$fit : +carvao : +supl : +carvao.supl

!

\$print 'TESTEMUNHA VS (AGUA + TIBA)'

$\$$ ca txs $=\%$ if $(\%$ eq $($ supl, 1$), 1,2)$

$\$$ factor txs 2

\$fit : +carvao : +txs : +txs.carvao

$!$

\$print 'AGUA VS TIBA'

$\$ v a r 20$ a b c d : 60 e f

$\$$ ca $a=0: e=1: f=2$

\$assign axt $=a, e, f, a, e, f$

$\$$ ca $w=\%$ if $(\%$ eq $($ axt, 0$), 0,1)$

$\$ c a$ axt $=\% \operatorname{if}(\%$ eq $($ axt, 0$), 1$, axt $)$

\$factor axt 2

\$wei w

\$fit : +carvao : +axt : +axt.carvao

!

\$print 'NIVEIS DE AGUA'

$\$ c a \quad a=0: b=1: c=2: d=3$

\$assign nia=a,b,c,d,a,a,a, a,b, c, d, a,a,a 
$\$ c a \mathrm{w}=\% \mathrm{if}(\%$ eq(nia,0),0,1)

$\$$ ca nia $=\%$ if $(\%$ eq $($ nia, 0$), 1$, nia $)$

Sfactor nia 3

\$wei w

\$fit : +carvao: +nia : +nia.carvao

!

\$print 'NIVEIS DE TIBA'

\$assign nit=a,a,a,a,b,c,d,a,a,a,a, b,c,d

$\$$ ca $w=\%$ if $(\%$ eq(nit, 0$), 0,1)$

$\$$ ca nit $=\%$ if $(\%$ eq(nit,0),1,nit $)$

\$factor nit 3

\$wei w

\$fit : +carvao : +nit : +nit.carvao

!

\$use bn

\$finish 


\section{APÊNDICE 8}

\section{Programa em GLIM para obtenção da "deviance" residual do modelo binomial negativo para 50 valores de $k$, aplicado ao Conjunto $B$ de dados}

$\$$ mac devi

$\$ \operatorname{var} 50 \mathrm{k} \mathrm{dev}$

$\$$ ca $k=\% \operatorname{gl}(50,1): \operatorname{dev}=\mathrm{k}: \mathrm{k}=\mathrm{k}+1: \mathrm{k}=\mathrm{k} / 20: \% \mathrm{c}=1$

\$while \%c ajuste

\$look k dev

$\$$ Sendmac

!

\$mac ajuste

$\$ c a \% \mathrm{k}=\mathrm{k}(\% \mathrm{c})$

\$yvar nc

\$own fv dr va di

$\$$ ca $y v=\%$ if $(\%$ eq $(\%$ yv, 0$), 0.001, \%$ yv $)$

$\$ c a \% l p=\% \log (y v /(y v+\% k))$

\$sca 1

\$fit \# model

\$assign sub $=\%$ c

$\$ c a \operatorname{dev}(\operatorname{sub})=\% d v$

$\$ c a \% c=\% c+1: \% c=\%$ if $(\% c<51, \% c, 0)$

$\$ \$$ endmac

!

$\$ m a c f v$

$\$$ ca $\%$ fv $=\% k^{*} \% \exp (\% \operatorname{lp}) /(1-\% \exp (\% \operatorname{lp})) \$ \$$ e

!

$\$ m a c d r$ 
$\$$ ca $\% \mathrm{dr}=\% \mathrm{k} /(\% \mathrm{fv} *(\% \mathrm{fv}+\% \mathrm{k})) \$ \$ \mathrm{e}$

!

$\$$ mac va

$\$ \mathrm{ca} \% \mathrm{va}=\% \mathrm{fv} *(1+(\% \mathrm{fv} / \% \mathrm{k})) \$ \$ \mathrm{e}$

!

$\$$ mac di

$\$ c a \%$ di $=2^{*}\left(\% \mathrm{yv}^{*}(\% \log (\mathrm{yv} / \% \mathrm{fv}))-(\% \mathrm{yv}+\% \mathrm{k}) * \% \log ((\% \mathrm{yv}+\% \mathrm{k}) /(\% \mathrm{fv}+\% \mathrm{k}))\right) \$ \$ \mathrm{e}$

!

\$mac model

\$fit : +carvao : +supl : +carvao.supl

$\$ \$$ endmac

!

Sunits 280

\$data nc

$\$$ read

$\begin{array}{rrrrrrrrrrrrrrrrrrrr}10 & 6 & 10 & 2 & 5 & 5 & 3 & 4 & 8 & 2 & 2 & 2 & 2 & 4 & 2 & 1 & 11 & 2 & 3 & 2 \\ 50 & 30 & 35 & 20 & 15 & 16 & 35 & 25 & 10 & 10 & 15 & 10 & 25 & 30 & 30 & 20 & 30 & 20 & 20 & 32 \\ 80 & 80 & 30 & 45 & 10 & 10 & 80 & 10 & 60 & 65 & 45 & 29 & 15 & 10 & 10 & 16 & 70 & 10 & 40 & 80 \\ 70 & 72 & 10 & 10 & 60 & 40 & 39 & 20 & 50 & 25 & 14 & 10 & 45 & 10 & 50 & 10 & 60 & 20 & 35 & 60 \\ 22 & 12 & 30 & 40 & 2 & 2 & 1 & 13 & 18 & 3 & 20 & 2 & 14 & 35 & 7 & 1 & 1 & 1 & 1 & 10 \\ 18 & 15 & 15 & 30 & 7 & 3 & 12 & 20 & 18 & 18 & 2 & 10 & 6 & 25 & 1 & 4 & 2 & 45 & 40 & 2 \\ 20 & 12 & 15 & 3 & 32 & 1 & 19 & 8 & 1 & 1 & 1 & 23 & 50 & 20 & 30 & 15 & 20 & 7 & 13 & 50 \\ 4 & 0 & 1 & 0 & 0 & 2 & 2 & 0 & 0 & 2 & 0 & 0 & 0 & 1 & 0 & 0 & 4 & 1 & 0 & 1 \\ 0 & 0 & 1 & 0 & 1 & 0 & 0 & 4 & 0 & 3 & 0 & 0 & 3 & 0 & 0 & 0 & 0 & 2 & 2 & 2 \\ 1 & 0 & 0 & 0 & 0 & 0 & 3 & 0 & 1 & 0 & 0 & 0 & 0 & 0 & 0 & 0 & 0 & 0 & 1 & 3 \\ 0 & 0 & 0 & 0 & 1 & 0 & 0 & 0 & 0 & 0 & 0 & 0 & 0 & 0 & 0 & 0 & 0 & 1 & 0 & 2 \\ 0 & 1 & 0 & 0 & 0 & 0 & 1 & 0 & 0 & 0 & 0 & 0 & 0 & 0 & 0 & 0 & 0 & 0 & 1 & 0 \\ 0 & 0 & 1 & 2 & 1 & 1 & 0 & 0 & 0 & 0 & 0 & 0 & 0 & 0 & 0 & 0 & 0 & 0 & 5 & 6 \\ 0 & 0 & 0 & 1 & 2 & 2 & 0 & 0 & 0 & 0 & 0 & 0 & 0 & 0 & 0 & 0 & 1 & 0 & 2 & 0\end{array}$

$\$$ ca carvao $=\% \mathrm{gl}(2,140): \operatorname{supl}=\% \mathrm{gl}(7,20)$

\$factor carvao 2 : supl 7

\$use devi \$finish 


\begin{abstract}
APÊNDICE 9
Programa em GLIM para o desdobramento das interações do modelo binomial negativo ajustado ao Conjunto $B$ de dados
\end{abstract}

$\$ \mathrm{mac} f \mathrm{v}$

sca $\%$ fv $=\%$ k*\%exp(\%lp) $/(1-\% \exp (\% l p)) \$ \$$ e

\$mac dr

$\$$ ca $\% \mathrm{dr}=\% \mathrm{k} /\left(\% \mathrm{fv}^{*}(\% \mathrm{fv}+\% \mathrm{k})\right) \$ \$ \mathrm{e}$

!

\$mac va

$\$$ ca $\%$ va $=\%$ fv* $(1+(\%$ fv $/ \% \mathrm{k})) \$ \$$ e

!

Smac di

$\$ \mathrm{ca} \% \mathrm{di}=2^{*}\left(\% \mathrm{yv}^{*}(\% \log (\mathrm{yv} / \% \mathrm{fv}))-(\% \mathrm{yv}+\% \mathrm{k}) * \% \log ((\% \mathrm{yv}+\% \mathrm{k}) /(\% \mathrm{fv}+\% \mathrm{k}))\right) \$ \$ \mathrm{e}$ !

\$units 280 \$data nc $\$$ read

$\begin{array}{rrrrrrrrrrrrrrrrrrrr}10 & 6 & 10 & 2 & 5 & 5 & 3 & 4 & 8 & 2 & 2 & 2 & 2 & 4 & 2 & 1 & 11 & 2 & 3 & 2 \\ 50 & 30 & 35 & 20 & 15 & 16 & 35 & 25 & 10 & 10 & 15 & 10 & 25 & 30 & 30 & 20 & 30 & 20 & 20 & 32 \\ 80 & 80 & 30 & 45 & 10 & 10 & 80 & 10 & 60 & 65 & 45 & 29 & 15 & 10 & 10 & 16 & 70 & 10 & 40 & 80 \\ 70 & 72 & 10 & 10 & 60 & 40 & 39 & 20 & 50 & 25 & 14 & 10 & 45 & 10 & 50 & 10 & 60 & 20 & 35 & 60 \\ 22 & 12 & 30 & 40 & 2 & 2 & 1 & 13 & 18 & 3 & 20 & 2 & 14 & 35 & 7 & 1 & 1 & 1 & 1 & 10 \\ 18 & 15 & 15 & 30 & 7 & 3 & 12 & 20 & 18 & 18 & 2 & 10 & 6 & 25 & 1 & 4 & 2 & 45 & 40 & 2 \\ 20 & 12 & 15 & 3 & 32 & 1 & 19 & 8 & 1 & 1 & 1 & 23 & 50 & 20 & 30 & 15 & 20 & 7 & 13 & 50 \\ 4 & 0 & 1 & 0 & 0 & 2 & 2 & 0 & 0 & 2 & 0 & 0 & 0 & 1 & 0 & 0 & 4 & 1 & 0 & 1 \\ 0 & 0 & 1 & 0 & 1 & 0 & 0 & 4 & 0 & 3 & 0 & 0 & 3 & 0 & 0 & 0 & 0 & 2 & 2 & 2 \\ 1 & 0 & 0 & 0 & 0 & 0 & 3 & 0 & 1 & 0 & 0 & 0 & 0 & 0 & 0 & 0 & 0 & 0 & 1 & 3 \\ 0 & 0 & 0 & 0 & 1 & 0 & 0 & 0 & 0 & 0 & 0 & 0 & 0 & 0 & 0 & 0 & 0 & 1 & 0 & 2 \\ 0 & 1 & 0 & 0 & 0 & 0 & 1 & 0 & 0 & 0 & 0 & 0 & 0 & 0 & 0 & 0 & 0 & 0 & 1 & 0 \\ 0 & 0 & 1 & 2 & 1 & 1 & 0 & 0 & 0 & 0 & 0 & 0 & 0 & 0 & 0 & 0 & 0 & 0 & 5 & 6 \\ 0 & 0 & 0 & 1 & 2 & 2 & 0 & 0 & 0 & 0 & 0 & 0 & 0 & 0 & 0 & 0 & 1 & 0 & 2 & 0\end{array}$

$\$ \operatorname{var} 140$ a b c 
$\$$ ca $a=\% \operatorname{gl}(7,20): b=a+10: c=a+20$

\$assign trat $=b, c$

\$del a b c

$\$$ ca $\operatorname{carvao}=\% \operatorname{gl}(2,140): \operatorname{supl}=\% \operatorname{gl}(7,20)$

\$factor carvao $2: \operatorname{supl} 7$

$\$ c a \% \mathrm{k}=1.5$

\$yvar nc

\$own fv dr va di

$\$$ ca $y v=\%$ if $(\%$ eq $(\% y v, 0), 0.001, \%$ yv $)$

$\$$ ca $\% \operatorname{lp}=\% \log (\mathrm{yv} /(\mathrm{yv}+\% \mathrm{k}))$

\$sca 1

\$fit : +carvao : +supl : +carvao.supl

!

\$print ' = DESDOBRAMENTO DAS INTERACOES ='

$!$

\$print ' - DENTRO DO NIVEL 1 DO FATOR CARVAO - '

!

\$print 'TESTEMUNHA VS (AGUA + TIBA)'

$\$$ ca $w=\%$ if $(\%$ eq $($ carvao, 2$), 0,1)$

$\$$ ca txs $=\%$ if(\%eq(supl,1),1,2)

\$factor txs 2

\$wei w

\$fit : + txs

!

\$print 'AGUA VS TIBA'

$\$ \operatorname{var} 20$ a b c d : 60 e f g

$\$ c a$ a $=0: e=1: f=2: g=0$

\$assign axt=a,e,f,a,g,g

$\$$ ca $\mathrm{w}=\%$ if $(\%$ eq $(\operatorname{axt}, 0), 0,1)$ 
$\$$ ca axt $=\%$ if $(\%$ eq $($ axt, 0$), 1$, axt $)$

\$wei w

\$factor axt 2

\$wei w

\$fit : +axt

!

\$print 'NIVEIS DE AGUA'

$\$ c a \quad a=0: b=1: c=2: d=3$

\$assign nia $=a, b, c, d, a, a, a, a, a, a, a, a, a, a$

$\$ c a \quad w=\%$ if(\%eq(nia,0),0,1)

$\$ c a$ nia $=\%$ if(\%eq(nia,0),1,nia)

$\$$ factor nia 3

\$wei w

\$fit : +nia

!

\$print 'NIVEIS DE TIBA'

$\$ c a b=1: c=2: d=3$

\$assign nit=a,a,a,a,b,c,d,a,a,a,a,a,a,a

$\$$ ca $w=\%$ if $(\%$ eq(nit, 0$), 0,1)$

$\$$ ca nit=\%if(\%eq(nit,0),1, nit)

\$factor nit 3

\$wei w

\$it : +nit

!

\$print ' - DENTRO DO NIVEL 2 DO FATOR CARVAO - '

!

\$print 'TESTEMUNHA VS (AGUA + TIBA)'

$\$$ ca $w=\%$ if $(\%$ eq $($ carvao, 1$), 0,1)$

\$wei w 
\$fit : +txs

!

\$print 'AGUA VS TIBA'

\$assign axt=a,g,g,a,e,f

$\$ c a$ w $=\%$ if $(\%$ eq $($ axt, 0$), 0,1)$

\$ca axt $=\%$ if $(\%$ eq $($ axt, 0$), 1$, axt $)$

\$factor axt 2

\$wei w

\$fit : +axt

!

\$print 'NIVEIS DE AGUA'

\$ca $a=0: b=1: c=2: d=3$

\$assign nia =a,a,a, a,a, a, a, a, b, c, d,a,a,a

$\$ c a$ w $=\%$ if(\%eq(nia, 0$), 0,1)$

$\$$ ca nia $=\%$ if(\%eq(nia, 0$), 1$, nia $)$

\$factor nia 3

\$wei w

\$fit : +nia

!

\$print 'NIVEIS DE TIBA'

$\$ c a b=1: c=2: d=3$

\$assign nit=a,a, a, a, a, a, a, a, a, a, a, , b,c,d

$\$$ ca $w=\%$ if(\%eq(nit,0),0,1)

$\$ c a$ nit=\%if(\%eq(nit,0),1,nit)

\$factor nit 3

\$wei w

\$fit : +nit

Sfinish 


\begin{abstract}
APÊNDICE 10
Programa em GLIM para desdobramento das interações, em efeitos linear e quadrático, do modelo binomial negativo ajustado ao Conjunto $B$ de dados
\end{abstract}

$\$ \mathrm{mac} f \mathrm{v}$

\$ca $\% \mathrm{fv}=\% \mathrm{k} * \% \exp (\% \mathrm{lp}) /(1-\% \exp (\% \mathrm{lp}))$

$\$$ ca $\% \mathrm{fv}=\%$ if( $\%$ eq( $\%$ fv, 0$), 0.01, \%$ fv $) \$ \$$ e

$!$

$\$ \operatorname{mac} \mathrm{dr}$

$\$ \mathrm{ca} \% \mathrm{dr}=\% \mathrm{k} /\left(\% \mathrm{fv}^{*}(\% \mathrm{fv}+\% \mathrm{k})\right) \$ \$ \mathrm{e}$

!

\$mac va

$\$ c a \% v a=\%$ fv* $*(1+(\%$ fv $/ \% \mathrm{k})) \$ \$$ e

!

$\$$ mac di

$\$$ ca $\% \mathrm{di}=2^{*}\left(\% \mathrm{yv}^{*}(\% \log (\mathrm{yv} / \% \mathrm{fv}))-(\% \mathrm{yv}+\% \mathrm{k}) * \% \log ((\% \mathrm{yv}+\% \mathrm{k}) /(\% \mathrm{fv}+\% \mathrm{k}))\right) \$ \$ \mathrm{e}$ !

\$units 280 \$data nc $\$$ read 


$\begin{array}{rrrrrrrrrrrrrrrrrrrr}10 & 6 & 10 & 2 & 5 & 5 & 3 & 4 & 8 & 2 & 2 & 2 & 2 & 4 & 2 & 1 & 11 & 2 & 3 & 2 \\ 50 & 30 & 35 & 20 & 15 & 16 & 35 & 25 & 10 & 10 & 15 & 10 & 25 & 30 & 30 & 20 & 30 & 20 & 20 & 32 \\ 80 & 80 & 30 & 45 & 10 & 10 & 80 & 10 & 60 & 65 & 45 & 29 & 15 & 10 & 10 & 16 & 70 & 10 & 40 & 80 \\ 70 & 72 & 10 & 10 & 60 & 40 & 39 & 20 & 50 & 25 & 14 & 10 & 45 & 10 & 50 & 10 & 60 & 20 & 35 & 60 \\ 22 & 12 & 30 & 40 & 2 & 2 & 1 & 13 & 18 & 3 & 20 & 2 & 14 & 35 & 7 & 1 & 1 & 1 & 1 & 10 \\ 18 & 15 & 15 & 30 & 7 & 3 & 12 & 20 & 18 & 18 & 2 & 10 & 6 & 25 & 1 & 4 & 2 & 45 & 40 & 2 \\ 20 & 12 & 15 & 3 & 32 & 1 & 19 & 8 & 1 & 1 & 1 & 23 & 50 & 20 & 30 & 15 & 20 & 7 & 13 & 50 \\ 4 & 0 & 1 & 0 & 0 & 2 & 2 & 0 & 0 & 2 & 0 & 0 & 0 & 1 & 0 & 0 & 4 & 1 & 0 & 1 \\ 0 & 0 & 1 & 0 & 1 & 0 & 0 & 4 & 0 & 3 & 0 & 0 & 3 & 0 & 0 & 0 & 0 & 2 & 2 & 2 \\ 1 & 0 & 0 & 0 & 0 & 0 & 3 & 0 & 1 & 0 & 0 & 0 & 0 & 0 & 0 & 0 & 0 & 0 & 1 & 3 \\ 0 & 0 & 0 & 0 & 1 & 0 & 0 & 0 & 0 & 0 & 0 & 0 & 0 & 0 & 0 & 0 & 0 & 1 & 0 & 2 \\ 0 & 1 & 0 & 0 & 0 & 0 & 1 & 0 & 0 & 0 & 0 & 0 & 0 & 0 & 0 & 0 & 0 & 0 & 1 & 0 \\ 0 & 0 & 1 & 2 & 1 & 1 & 0 & 0 & 0 & 0 & 0 & 0 & 0 & 0 & 0 & 0 & 0 & 0 & 5 & 6 \\ 0 & 0 & 0 & 1 & 2 & 2 & 0 & 0 & 0 & 0 & 0 & 0 & 0 & 0 & 0 & 0 & 1 & 0 & 2 & 0\end{array}$

$\$ \operatorname{var} 140$ a b c

$\$ c a \quad a=\% g l(7,20): b=a+10: c=a+20$

\$assign trat $=b, c$

\$del a b c

$\$$ ca $\operatorname{carvao}=\% \mathrm{gl}(2,140): \operatorname{supl}=\% \mathrm{gl}(7,20)$

\$factor carvao $2: \operatorname{supl} 7$

$\$$ ca $\% \mathrm{k}=1.5$

\$yvar nc

\$own fv dr va di

$\$$ ca yv $=\%$ if $(\%$ eq $(\% y v, 0), 0.001, \% y v)$

$\$ c a \% \operatorname{lp}=\% \log (\mathrm{yv} /(\mathrm{yv}+\% \mathrm{k}))$

\$sca 1

\$fit : +carvao : +supl : +carvao.supl

!

\$print ' = REGRESSAO PARA NIVEIS DE AGUA = '

\$print ' = DENTRO DO NIVEL 1 DO FATOR CARVAO = ' 
$\$ \operatorname{var} 20$ a b c d

$\$ c a \quad a=0: b=5: c=10: d=15$

\$assign nia $=\mathrm{a}, \mathrm{b}, \mathrm{c}, \mathrm{d}, \mathrm{a}, \mathrm{a}, \mathrm{a}, \mathrm{a}, \mathrm{a}, \mathrm{a}, \mathrm{a}, \mathrm{a}, \mathrm{a}, \mathrm{a}$

$\$$ ca $w=\%$ if $(\%$ eq(nia, 0$), 0,1)$

$\$$ ca nia $=\%$ if(\%eq(nia, 0$), 1$, nia $)$

$\$ c a$ niaq=nia*nia

\$wei w

\$fit : +nia : +niaq

!

\$print ' = REGRESSAO PARA NIVEIS DE TIBA = '

$\$$ print ' $=$ DENTRO DO NIVEL 1 DO FATOR CARVAO ='

$!$

$\$ c a b=0.05: c=0.10: d=0.20$

\$assign nit=a,a,a,a,b,c,d,a,a,a,a,a,a,a

$\$ c a$ w $=\%$ if(\%eq(nit,0),0,1)

$\$$ ca nit $=\%$ if $(\%$ eq(nit,0),1,nit $)$

\$ca nitq=nit*nit

\$wei w

\$fit : +nit : +nitq

!

\$print ' = REGRESSAO PARA NIVEIS DE AGUA = '

$\$$ print ' = DENTRO DO NIVEL 2 DO FATOR CARVAO = '

!

$\$ c a b=5: c=10: d=15$

\$assign nia $=\mathrm{a}, \mathrm{a}, \mathrm{a}, \mathrm{a}, \mathrm{a}, \mathrm{a}, \mathrm{a}, \mathrm{a}, \mathrm{b}, \mathrm{c}, \mathrm{d}, \mathrm{a}, \mathrm{a}, \mathrm{a}$

$\$ c a$ w $=\%$ if(\%eq(nia, 0$), 0,1)$

$\$$ ca nia $=\%$ if $(\%$ eq(nia, 0$), 1$, nia $)$

\$ca niaq=nia*nia

\$wei w 
\$fit : +nia : +niaq

$\$$ fit : +nia

\$display e

\$look \%lp \%fv

$!$

\$print ' = REGRESSAO PARA NIVEIS DE TIBA = '

$\$$ print $'=$ DENTRO DO NIVEL 2 DO FATOR CARVAO ='

!

$\$ c a b=0.05: c=0.10: d=0.20$

\$assign nit=a,a, a, a,a, a, a, a, a,a a, a,b,c,d

$\$$ ca $w=\%$ if $(\%$ eq(nit, 0$), 0,1)$

$\$ c a$ nit $=\%$ if $(\%$ eq $($ nit, 0$), 1$, nit $)$

\$ca nitq=nit*nit

\$wei w

\$fit : +nit : +nitq

\$look \%lp \%fv

\$finish 


\section{APÊNDICE 11}

Macros para construção, no GLIM, do gráfico normal de probabilidade para os valores absolutos dos componentes de "deviance" padronizados com envelope simulado, para o modelo Poisson

\$mac hnp

$\$$ ca $\% \mathrm{z} 2=\% \mathrm{z} 3=1: \mathrm{i}=\% \mathrm{cu}(1)$

\$use res rd

\$sort rd

$\$$ ca $\min =\max =\operatorname{med}=0$

\$while \%z2 env

$\$$ ca nd $=\%$ nd $((i+\%$ nu-0.125) $/(2 * \%$ nu +0.5$))$

\$plot min med max rd nd '-..*',

\$look min med max rd nd

$\$$ del i hi dn yv rd1 n s p \%c \%r rp u k p t v

\$ \$endmac

!

$\$$ mac res

\$ext \%vl

$\$$ ca hi $=\%$ vl $\%$ wt $:$ dn $=\%$ sqrt $(1-h i)$

$\$$ ca $y v=\%$ if $(\%$ eq $(\%$ yv, 0$), 0.001, \%$ yv $)$

$\$$ ca $\% 1=2^{*}\left(\left(\% \mathrm{yv}^{*} \% \log (\mathrm{yv} / \% \mathrm{fv})\right)-\% \mathrm{yv}+\% \mathrm{fv}\right)$

\$ca $\% 1=\% \operatorname{sqrt}(\% 1): \% 1=\% 1 / \mathrm{dn}$

\$endmac

!

\$mac env

\$use gen

$\$ y$ var rp

\$error $p$ 
\$link 1

\$sca 1

\$fit! declarar o modelo !

\$use res rd1

\$sort rd1

$\$$ ca $\min =\%$ if $(\%$ eq $(\% \mathrm{z} 3,1), \mathrm{rd} 1, \mathrm{~min}):$

$$
\begin{aligned}
& \operatorname{med}=\% \operatorname{if}(\% \mathrm{eq}(\% \mathrm{z} 3,1), \mathrm{rd} 1 / 19, \operatorname{med}+(\mathrm{rd} 1 / 19)): \\
& \max =\% \operatorname{if}(\% \mathrm{eq}(\% \mathrm{z} 3,1), \mathrm{rd} 1, \max ): \\
& \min =\% \operatorname{if}(\% \mathrm{lt}(\min , \mathrm{rd} 1), \min , \mathrm{rd} 1): \\
& \max =\% \operatorname{if}(\% \operatorname{gt}(\max , \mathrm{rd} 1), \max , \mathrm{rd} 1): \\
& \% \mathrm{z} 3=\% \mathrm{z} 3+1: \\
& \% \mathrm{z} 2=\% \mathrm{z} 3<20
\end{aligned}
$$

$\$ y v a r$ nc

\$error $p$

$\$$ link 1

\$sca 1

\$fit ! declarar o modelo !

$\$ \$$ endmac

!

\$mac gen

$\$ c a n=0: s=0: p=1: \% c=1: \% r=1$

$\$$ wh \%r poi

$\$$ ca $\mathrm{rp}=\mathrm{s}$

$\$ \$$ endmac

!

\$mac poi

$\$$ ca $u=\% \operatorname{sr}(0)$

\$print 'PASSO '\%c

\$ca $\mathrm{k}=(-1 / \% \mathrm{fv}) * \% \log (\mathrm{u})$ 
$\$$ ca $p=\%$ iff(\%eq(p, 0$), 0, k)$

$\$ c a t=\%$ if $(\% \operatorname{gt}(p, 1), n, s)$

\$ca $\mathrm{p}=\%$ if $(\% \operatorname{gt}(\mathrm{p}, 1), 0, \mathrm{p})$

$\$$ ca $v=\%$ if $(\%$ eq $(p, 0), 0,(p+s))$

$\$$ ca $s=\mathrm{t}+\mathrm{p}: \mathrm{s}=\% \operatorname{if}(\% \operatorname{gt}(\mathrm{v}, 1), \mathrm{n}, \mathrm{s})$

$\$$ ca v $=\%$ if(\%gt(v,1),0,v) : p=\%if(\%eq(v,0),0,p)

$\$ c a n=n+1: \% c=\% c+1: \% r=\% c u(p)$

$\$$ print $\% \mathrm{R}=$ '\%

$\$ \$$ endmac 


\section{APÊNDICE 12}

Tabela 19: Tratamentos do Conjunto A de dados ordenados pelas médias do número de calos produzidos por explante, e número de repetições dos tratamentos $(n)$.

\begin{tabular}{cccc}
\hline & Tratamento & Média & $n$ \\
\hline 1 & 32 & 0,00 & 14 \\
2 & 33 & 0,00 & 14 \\
3 & 13 & 0,14 & 14 \\
4 & 23 & 0,14 & 14 \\
5 & 31 & 0,28 & 14 \\
6 & 22 & 0,36 & 14 \\
7 & 12 & 0,86 & 14 \\
8 & 21 & 9,00 & 15 \\
9 & 11 & 10,07 & 14 \\
\hline
\end{tabular}




\section{APÊNDICE 13}

Tabela 20: Tratamentos do Conjunto A de dados ordenados pelas médias do número de calos produzidos por explante, dado que o explante regenerou, e número de repetições dos tratamentos $(n)$.

\begin{tabular}{lccc}
\hline & Tratamento & Média & $n$ \\
\hline 1 & 13 & 1,00 & 2 \\
2 & 31 & 1,33 & 3 \\
3 & 12 & 1,71 & 7 \\
4 & 23 & 2,00 & 1 \\
5 & 22 & 2,50 & 2 \\
6 & 21 & 9,00 & 15 \\
7 & 11 & 10,07 & 14 \\
\hline
\end{tabular}




\section{APÊNDICE 14}

Tabela 21: Tratamentos do Conjunto B de dados ordenados pelas médias do número de calos produzidos por explante, e número de repetições dos tratamentos $(n)$.

\begin{tabular}{rccc}
\hline & Tratamento & Média & $n$ \\
\hline 1 & 25 & 0,15 & 20 \\
2 & 24 & 0,20 & 20 \\
3 & 27 & 0,40 & 20 \\
4 & 23 & 0,45 & 20 \\
5 & 26 & 0,80 & 20 \\
6 & 21 & 0,90 & 20 \\
7 & 22 & 0,90 & 20 \\
8 & 11 & 4,30 & 20 \\
9 & 15 & 11,75 & 20 \\
10 & 16 & 14,65 & 20 \\
11 & 17 & 17,05 & 20 \\
12 & 12 & 23,90 & 20 \\
13 & 14 & 35,50 & 20 \\
14 & 13 & 39,75 & 20 \\
\hline
\end{tabular}




\section{APÊNDICE 15}

Tabela 22: "Deviance" residual do modelo binomial negativo ajustado ao Conjunto B de dados, para diferentes valores do parâmetro $k$. (Saída do programa apresentado no Apêndice 8).

\begin{tabular}{ccc}
\hline Ajuste & $k$ & "Deviance" média \\
\hline 1 & 0,10 & 53,81 \\
2 & 0,15 & 70,39 \\
3 & 0,20 & 84,61 \\
4 & 0,25 & 97,24 \\
5 & 0,30 & 108,69 \\
6 & 0,35 & 119,24 \\
7 & 0,40 & 129,06 \\
8 & 0,45 & 138,22 \\
9 & 0,50 & 147,01 \\
10 & 0,55 & 155,30 \\
11 & 0,60 & 163,22 \\
12 & 0,65 & 170,81 \\
13 & 0,70 & 178,12 \\
14 & 0,75 & 185,16 \\
15 & 0,80 & 191,97 \\
16 & 0,85 & 198,57 \\
17 & 0,90 & 204,98 \\
18 & 0,95 & 211,20 \\
19 & 1,00 & 217,26 \\
20 & 1,05 & 223,18 \\
\hline & & $\ldots$ continua \\
& &
\end{tabular}


Tabela 22: "Deviance" residual do modelo binomial negativo ajustado ao Conjunto $\mathrm{B}$ de dados, para diferentes valores do parâmetro $k$. (Saída do programa apresentado no Apêndice 8). (continuação)

\begin{tabular}{ccc}
\hline Ajuste & $k$ & "Deviance" média \\
\hline 21 & 1,10 & 228,94 \\
22 & 1,15 & 234,59 \\
23 & 1,20 & 240,11 \\
24 & 1,25 & 245,51 \\
25 & 1,30 & 250,80 \\
26 & 1,35 & 256,00 \\
27 & 1,40 & 261,10 \\
28 & 1,45 & 266,10 \\
29 & 1,50 & 271,02 \\
30 & 1,55 & 275,86 \\
31 & 1,60 & 280,62 \\
32 & 1,65 & 285,30 \\
33 & 1,70 & 289,91 \\
34 & 1,75 & 294,46 \\
35 & 1,80 & 298,94 \\
36 & 1,85 & 303,35 \\
37 & 1,90 & 307,70 \\
38 & 1,95 & 312,00 \\
39 & 2,00 & 316,24 \\
40 & 2,05 & 320,42 \\
\hline & & $\ldots$ continua \\
& &
\end{tabular}


Tabela 22: "Deviance" residual do modelo binomial negativo ajustado ao Conjunto $\mathrm{B}$ de dados, para diferentes valores do parâmetro $k$. (Saída do programa apresentado no Apêndice 8). (continuação)

\begin{tabular}{ccc}
\hline Ajuste & $k$ & "Deviance" média \\
\hline 41 & 2,10 & 324,56 \\
42 & 2,15 & 328,64 \\
43 & 2,20 & 332,67 \\
44 & 2,25 & 336,66 \\
45 & 2,30 & 340,59 \\
46 & 2,35 & 344,49 \\
47 & 2,40 & 348,34 \\
48 & 2,45 & 352,15 \\
49 & 2,50 & 355,92 \\
50 & 2,55 & 359,65 \\
\hline
\end{tabular}




\section{APÊNDICE 16}

Tabela 23: Médias do número de calos produzidos por explante $(m)$ para as placas, ou repetições, dos tratamentos do Conjunto $\mathrm{C}$ de dados.

\begin{tabular}{rccc}
\hline & Placa & Tratamento & $m$ \\
\hline 1 & 1 & 11 & 2,1 \\
2 & 2 & 11 & 3,1 \\
3 & 3 & 11 & 3,6 \\
4 & 4 & 11 & 4,1 \\
5 & 5 & 11 & 3,1 \\
6 & 1 & 21 & 1,8 \\
7 & 2 & 21 & 0,5 \\
8 & 3 & 21 & 0,0 \\
9 & 4 & 21 & 0,2 \\
10 & 1 & 31 & 1,6 \\
11 & 2 & 31 & 1,9 \\
12 & 3 & 31 & 1,8 \\
13 & 4 & 31 & 6,5 \\
14 & 1 & 41 & 0,0 \\
15 & 2 & 41 & 0,7 \\
16 & 3 & 41 & 0,0 \\
17 & 4 & 41 & 0,0 \\
18 & 1 & 12 & 4,3 \\
19 & 2 & 12 & 2,8 \\
20 & 3 & 12 & 1,6 \\
\hline
\end{tabular}


Tabela 23: Médias do número de calos produzidos por explante $(m)$ para as placas, ou repetições, dos tratamentos do Conjunto $\mathrm{C}$ de dados. (continuação)

\begin{tabular}{lccc}
\hline & Placa & Tratamento & $m$ \\
\hline 21 & 4 & 12 & 2,2 \\
22 & 1 & 22 & 0,4 \\
23 & 2 & 22 & 0,0 \\
24 & 3 & 22 & 0,0 \\
25 & 4 & 22 & 0,3 \\
26 & 1 & 32 & 6,8 \\
27 & 2 & 32 & 7,2 \\
28 & 3 & 32 & 0,0 \\
29 & 4 & 32 & 0,3 \\
30 & 1 & 42 & 0,0 \\
31 & 2 & 42 & 0,0 \\
32 & 3 & 42 & 0,0 \\
33 & 4 & 42 & 0,0 \\
34 & 1 & 13 & 1,9 \\
35 & 2 & 13 & 2,6 \\
36 & 3 & 13 & 2,0 \\
37 & 4 & 13 & 2,0 \\
38 & 1 & 23 & 0,6 \\
39 & 2 & 23 & 0,0 \\
40 & 1 & 33 & 5,5 \\
\hline & & &
\end{tabular}


Tabela 23: Médias do número de calos produzidos por explante $(m)$ para as placas, ou repetições, dos tratamentos do Conjunto $\mathrm{C}$ de dados. (continuação)

\begin{tabular}{cccc}
\hline & Placa & Tratamento & $m$ \\
\hline 41 & 1 & 43 & 1,3 \\
42 & 2 & 43 & 1,2 \\
43 & 3 & 43 & 2,1 \\
44 & 4 & 43 & 0,1 \\
\hline
\end{tabular}




\section{APÊNDICE 17}

Tabela 24: Tratamentos do Conjunto $\mathrm{C}$ de dados ordenados pelas médias do número de calos produzidos por explante, e número de repetições dos tratamentos $(n)$.

\begin{tabular}{rccc}
\hline & Tratamento & Média & $n$ \\
\hline 1 & 42 & 0,00 & 4 \\
2 & 41 & 0,18 & 4 \\
3 & 22 & 0,18 & 4 \\
4 & 23 & 0,30 & 2 \\
5 & 21 & 0,62 & 4 \\
6 & 43 & 1,18 & 4 \\
7 & 13 & 2,12 & 4 \\
8 & 12 & 2,72 & 4 \\
9 & 31 & 2,95 & 4 \\
10 & 11 & 3,20 & 5 \\
11 & 32 & 3,58 & 4 \\
12 & 33 & 5,50 & 1 \\
\hline
\end{tabular}

